Annals of Mathematics, 155 (2002), 611-708

\title{
Complete moduli in the presence of semiabelian group action
}

\author{
By Valery Alexeev
}

\begin{abstract}
I prove the existence, and describe the structure, of moduli space of pairs $(P, \Theta)$ consisting of a projective variety $P$ with semiabelian group action and an ample Cartier divisor on it satisfying a few simple conditions. Every connected component of this moduli space is proper. A component containing a projective toric variety is described by a configuration of several polytopes, the main one of which is the secondary polytope. On the other hand, the component containing a principally polarized abelian variety provides a moduli compactification of $A_{g}$. The main irreducible component of this compactification is described by an "infinite periodic" analog of the secondary polytope and coincides with the toroidal compactification of $A_{g}$ for the second Voronoi decomposition.
\end{abstract}

\section{Contents}

Introduction

Section 1. Overview

1.1. Fundamental definitions
A. Stable toric and semiabelic varieties
B. The relative case
C. Complexes of cones and lattice polytopes

1.2. Main results

A. Moduli problem: abelic case

B. Classification of stable toric varieties and pairs over closed fields

C. Classification of SSAVs and pairs over closed fields

D. Classification of SSAVs and pairs over $\mathbb{C}$

E. Cohomologies of polarized stable semiablic varieties

F. Types and singularities of SSAVs

G. Moduli problem: the finite case

H. Moduli problem: the infinite periodic case

Section 2. Stable toric varieties and pairs

2.1. Complexes, posets and (co)sheaves on them

2.2. Basic (co)sheaves and relations between them

2.3. Affine stable toric varieties

2.4. Polarized stable toric varieties with a linearized line bundle 
2.5. Cohomologies of polarized STVs

2.6. Stable toric pairs

2.7. The moment map for stable toric pairs over $\mathbb{C}$

2.8. One-parameter families of stable toric pairs

2.9. The modified complex $\mathbb{M}_{*}$

2.10. Moduli of stable toric pairs

2.11. Approximation of the moduli space

2.12. Generalized secondary polytopes for finite complexes

2.13. The moment map for the moduli space

2.14. Relation to families of Gelfand-Kapranov-Zelevinsky

2.15. Other families

2.16. First nontrivial example

Section 3. Abelic pairs

Section 4. Linearization of torus action

4.1. The polarization morphism and theorem of square

A. The case of a closed field

B. The case of families

4.2. Tori in Picard groups and infinite covers

4.3. Infinite covers arising from the torus action

Section 5. Stable semiabelic varieties and pairs

5.1. (Co)sheaves on general cell complexes

5.2. Linearized varieties with a nontrivial abelian part

5.3. Arbitrary polarized SSAVs

5.4. Arbitrary stable semiabelic pairs

5.5. Stable semiabelic pairs over $\mathbb{C}$

5.6. Mumford-Faltings-Chai's uniformization of abelian varieties

5.7. One-parameter families of stable semiabelic pairs

5.8. The modified complex $\mathbb{M}_{*}$

5.9. Test families of stable semiabelic pairs

A. The totally degenerate case: $\operatorname{dim} \Delta^{\prime}=g$

B. The general case

5.10. Moduli of stable semiabelic pairs

5.11. Approximation of the moduli space

5.12. Generalized secondary polytopes for periodic decompositions

5.13. Periodic non-Delaunay decompositions

Section 6. Further questions

References

\section{Introduction}

In this paper I construct a proper moduli stack and its coarse moduli space, also proper, of pairs $(P, \Theta)$, where $P$ is a reduced projective scheme with a semiabelian group action and $\Theta$ is a Cartier divisor. The pairs should satisfy a few simple conditions. For me, the motivation for considering moduli 
of such pairs came from the log minimal model program; see [Ale2]. However, the minimal model program in dimensions $\geq 4$ remains conjectural, and so in this paper I do not use it.

When the group acting on our variety is a torus and the sheaf $\mathcal{O}_{P}(\Theta)$ is linearized, our theory is closely related to the Gelfand-Kapranov-Zelevinsky study of families of cycles and their toric degenerations; see [GKZ] and references there. (The cycles appear as images of our pairs under a natural projection to $\mathbb{P}^{n}$.) The notions of regular decompositions and secondary polytopes defined there play an important role in our study. It turns out the closure of the stratum containing the standard projective toric variety $P[Q]$ for a lattice polytope $Q$ is itself the projective toric variety corresponding to the secondary polytope $\Sigma(Q)$. Examination of the other strata in our moduli space naturally leads to definition of the generalized secondary polytopes for each subdivision of $Q$. I describe an explicit stratification on the space and compute the dimensions of the strata. Their exact configuration depends on the resolution of the so-called generalized Baues problem in geometric combinatorics. (I thank Professor Sturmfels for pointing out this connection to me.)

One of the most interesting strata in our moduli space is the one corresponding to abelian torsors with a divisor defining principal polarization. This stratum can be naturally identified with $A_{g}$, the moduli of principally polarized abelian varieties. Since our moduli is proper, we obtain a geometric way of compactifying $A_{g}$, with every point on the boundary corresponding to a simple projective object. The ideas of the toric case turn out to apply here. The analog of a regular decomposition is the so-called Delaunay decomposition. I also construct the corresponding "infinite GL-invariant" secondary polytope. The closure of $A_{g}$ is the toroidal compactification for the second Voronoi cone decomposition. As a consequence of our construction with the polytope, it is projective, which was apparently unknown until now. We note that for a higher degree of polarization $d$ there is a stratum in our moduli which is fibered over $A_{g, d}$ with fibers of dimension $d-1$. There should be a way of looking at only some of the pairs to get a space with a finite morphism to $A_{g, d}$. There should also be a way to define appropriate level structures for our pairs. We will deal with these questions elsewhere.

According to the stable reduction theorem, when abelian varieties degenerate, the limit is a semi-abelian group variety which is an extension of an abelian variety by a torus. Therefore, the situation near the boundary of abelian moduli and the toric situation have a lot in common. The main idea of this work is to understand everything first in the "finite" case where it is quite simple and then, when working in the "infinite periodic" case, exploit the analogy to the maximum.

In the "finite" case, i.e. of pairs with a torus action and linearized $\mathcal{O}_{P}(\Theta)$, the moduli are constructed directly, bypassing any deformation theory. The 
moduli stack of such toric pairs is glued directly from several smooth groupoids $[U / R]$ with finite stabilizers. In this case $U$ is the spectrum of an explicit semigroup algebra, and $R$ is given by a torus action. Hence, we get an Artin stack and it is coarsely represented by an algebraic space according to [KM]. Moreover, in our case this space turns out to be a projective scheme, again with an explicit combinatorial description.

In the "infinite periodic" case, the one corresponding to abelian torsors and their degenerations, the situation is a little more complicated because in a semi-abelian family the toric rank may vary. However, for as long as we only look at an infinitesimal family over $S$, the toric rank stays constant and the semi-abelian group scheme $G / S$ is a global extension of an abelian scheme $A / S$ by a torus $T / S$. These families we can describe by the same toric methods and semigroup algebras as before. Hence, we get all the deformation theory essentially "for free" here. To prove that our moduli stack is an algebraic Artin stack with finite stabilizer (again, coarsely representable by an algebraic space by $[\mathrm{KM}]$ ) we use Artin's method in [Art2]. Our basic method in this case, therefore, is very similar to that of Faltings and Chai $[\mathrm{FC}]$ but with the following difference. The considerations of [FC, IV.3, IV.4] are very delicate because they did not have an easily definable functor and could not quote M. Artin directly. Our considerations are not so delicate because we do and we can. We note that once one toroidal compactification is constructed, all others can be constructed from it by blowing up and down on the boundary and, for moduli with level structures, also looking at finite covers and normalizing.

The subject of compactifications of moduli of abelian varieties has a very rich history which we will not attempt to summarize here. Some directly relevant papers, aside from those already mentioned are: [AMMT], [Mum1], [Nam1,2].

About the base scheme, there was not much to gain by working over a (closed) field $k$; much of our work consists of working with semigroups and semigroup algebras, and a semigroup algebra $\mathbb{Z}[H]$ is just as simple as semigroup algebra $k[H]$. A toric scheme over $\mathbb{Z}$ corresponding to a cone or a polytope is just as simple as the similar toric variety over $k$. Moduli of abelian varieties also exist over $\mathbb{Z}$. Hence, for most of this paper we work over $\mathbb{Z}$. One can make obvious changes to modify our arguments and notation for the case of a field or some other scheme.

Acknowledgements. I am indebted to many people with whom I interacted during the work on this paper. It started after a suggestion made to me by Y. Kawamata concerning my article on the two-dimensional case. Collaboration with I. Nakamura on [AN] was a very important first step. Discussions with J. Kollár have helped me to crystallize the definition of the functor. I have also profited greatly from discussions with F. Bogomolov about 
theorem of square and with R. Erdahl about Delaunay decompositions. I had an opportunity to talk about parts of this work as well with R. Varley, G. Faltings, M. Kapranov, H. Lenstra, L. Moret-Bailly, S. Mori, T. Oda, F. Oort, W. J. Whiteley and my colleagues at the University of Georgia. I am very grateful for their suggestions. I also thank T. Kajiwara and T. Ueno for pointing out some misprints.

I would also like to thank RIMS, Kyoto University, where part of this work was done, for hospitality and an excellent research environment, as well as NSF and the J. P. Sloan Foundation for providing partial support.

\section{Overview}

\subsection{Fundamental definitions.}

A. Stable toric and semiabelic varieties. Fix an algebraically closed field $k$ of arbitrary characteristic. By a variety over $k$ we will mean a connected reduced but not necessarily irreducible separated scheme of finite type over $k$. Recall the following:

Definition 1.1.1. 1. A multiplicative torus is a direct sum of several copies of the multiplicative group of the field $k: T=\mathbb{G}_{m}^{r}$.

2. An abelian variety is a (connected) proper group variety $A$ over $k$. It is then necessarily commutative and projective.

3. A semiabelian variety is a group variety which is an extension

$$
1 \rightarrow T \rightarrow G \rightarrow A \rightarrow 0
$$

of an abelian variety $A$ by a torus $T$. It is automatically commutative as well. Such extensions are classified by homomorphisms $c: X \rightarrow A^{t}$ from the group of characters of $T$ to the dual abelian variety of $A$.

Definition 1.1.2. Let $L$ be an invertible sheaf on an abelian variety $A$. A polarization morphism $\lambda(L): A \rightarrow A^{t}=\mathbf{P i c}^{0} A$ is defined by $a \mapsto T_{a}^{*} L \otimes$ $L^{-1}$. It is a homomorphism by theorem of square. A polarization on $A$ is a homomorphism $\lambda: A \rightarrow A^{t}$ which equals $\lambda(L)$ for some ample $L$. The sheaf $L$ with this property is not unique, and two such sheaves differ by $E \in \operatorname{Pic}^{0} P$. The degree of polarization is defined as $d=h^{0}(L)=\sqrt{\operatorname{deg} \lambda}$.

Definition 1.1.3. 1. By a toric variety we will mean a variety $P$ together with an action of a torus $T$ such that

(i) $P$ is normal, 
(ii) there are only finitely many orbits.

2. By analogy, a semi-abelic variety is a variety $P$ together with an action of a semi-abelian variety $G$ satisfying the same basic conditions.

We will add the following mild condition on the action, both for toric and semiabelic cases:

The stabilizer of the generic point is connected, reduced and lies in the toric part $T$ of $G$ (we view it as a group scheme over $k$ ).

3. If $G=A$ is abelian, we will call $P$ an abelic variety.

Remarks 1.1.4. 1) We will contrast toric varieties with torus embeddings $P \supset T$. The difference is that for us a toric variety need not have an origin $1 \in T \subset P$ fixed, and the torus action need not be faithful. However, if $T \curvearrowright P$ is a toric variety in our definition, $T^{\prime}$ is the quotient of $T$ by the stabilizer of the generic point and $p \in P$ is an arbitrary point in the dense orbit, then by sending $1 \in T^{\prime}$ to $p$ we obtain the structure of a torus embedding $P \supset T^{\prime}$.

2) Since we assumed $P$ to be normal, it follows that the condition (iii) above holds for every point $p \in P$.

3) In the abelian case there is already a perfectly good name "abelian torsor". We will use the term "abelic variety" in the interests of uniformity. Unlike the toric case, allowing the action to be nonfaithful does not add any flexibility here, so we will forgo it.

I thank S. Keel for suggesting the word "semi-abelic".

Definition 1.1.5. A stable semiabelic variety, or SSAV for short, is a variety $P$ together with an action of a semiabelian variety $G$ of the same dimension such that:

(i) $P$ is seminormal.

(ii) There are only finitely many orbits.

(iii) The stabilizer of every point is connected, reduced and lies in the toric part $T$ of $G$.

Definition 1.1.6. A reduced scheme $P$ is called seminormal if every proper bijective morphism $f: P^{\prime} \rightarrow P$ with reduced $P^{\prime}$ inducing isomorphisms on the residue fields $\kappa\left(p^{\prime}\right) \supset \kappa(p)$ for each $p \in P$ is an isomorphism. A reduced scheme $P$ is called weakly normal if every proper bijective morphism $f: P^{\prime} \rightarrow P$ inducing isomorphisms $\kappa\left(p^{\prime}\right) \supset \kappa(p)$ for each generic point $p \in P$ and a purely nonseparable extension for every other point is an isomorphism. 
Every reduced scheme with a finite normalization (i.e. $P$ is of finite type over a field or $\mathbb{Z}$ or a localization of such) has unique seminormalization and weak normalization. They coincide if $\operatorname{char} k=0$. See [Kol, I.7.2] for an overview of their properties. [GT] contains a wealth of information about seminormal schemes.

We will see that our varieties are weakly normal as well.

Remark 1.1.7. Following the tradition that started with stable curves, we call our most general varieties stable semiabelic varieties. Note that a stable semiabelic variety need not be plainly semiabelic; in other words, stable acts as a widening term here, not a narrowing one.

Definition 1.1.8. A polarized stable semiabelic variety is a projective SSAV together with an ample invertible sheaf $L$. The degree of polarization is defined as $h^{0}(L)$.

Definition 1.1.9. A stable semiabelic pair $(P, \Theta)$ consists of a stable projective semiabelic variety $P$ and an effective ample Cartier divisor $\Theta$ on it which does not contain any $G$-orbits. $P$ is polarized by $L=\mathcal{O}_{P}(\Theta)$.

Semiabelic, abelic, toric and stable toric pairs are defined in the same way.

B. The relative case. Instead of a closed field, we now work over an arbitrary base scheme $S$. We need to recall the basics about semiabelian group schemes. The references for this are [SGA3, X], [Mum2, Ch. 6], [FC, Ch. I].

Definition 1.1.10. 1. A split (other names: constant or diagonalizable) torus of rank $r$ over a scheme $S$ is a fibered product over $S$ of several copies of $\mathbb{G}_{m, S}$. A torus $T$ of rank $r$ over $S$ is a smooth group scheme over $S$ which locally in fppf topology is a split torus of rank $r$. In other words, there exists a covering family $\left\{U_{i} \rightarrow S\right\}$ of flat morphisms of finite presentation such that $T \underset{S}{\times} U_{i} \simeq \mathbb{G}_{m, U_{i}}^{r}$. In this definition, fppf can be replaced by étale.

Equivalently, $T$ is a smooth separated group scheme of finite presentation over $S$ whose every geometric fiber is a torus of rank $r$ ([SGA3, XV.8.18], [FC, I.2.11]).

2. An abelian scheme over $S$ is a proper smooth group scheme $A$ with connected fibers. It is automatically commutative.

3. A semiabelian scheme over $S$ is a smooth separated group scheme over $S$ such that every geometric fiber $G_{\bar{s}}$ is a semiabelian variety over $\kappa(\bar{s})$. It is commutative by [SGA3, XVI.1.6]. 
Since the formation of maximal tori in algebraic groups is compatible with field extensions ([SGA3, XIV.1.1]), a semiabelian group scheme over the spectrum of a nonclosed field $k^{\prime}$ is an extension over $k^{\prime}$ of a torus $T$ by an abelian variety $A$. For any base $S$, if the dimensions of abelian and toric parts of $G / S$ are constant then $G$ is a global extension of some abelian scheme $A$ by a torus $T$ over $S$ ([FC, I.2.11]).

Definition 1.1.11. A polarization on an abelian scheme is a homomorphism $\lambda: A \rightarrow A^{t}$ to the dual abelian scheme $A^{t}=\mathbf{P i c}_{A / S}^{0}$ whose restriction to every geometric fiber $A_{\bar{s}}$ is induced by an ample sheaf $L_{\bar{s}}$.

Remark 1.1.12. The sheaf $L$ need not exist globally.

Definition 1.1.13. A stable semiabelic scheme over a scheme $S$ is a flat separated scheme $P$ of finite presentation over $S$ together with an action by a semiabelian group scheme $G / S$ such that every geometric fiber is a stable semiabelic variety over $\kappa(\bar{s})$.

Definition 1.1.14. A polarized stable semiabelic scheme over a scheme $S$ is a stable semiabelic scheme $G \curvearrowright P$ proper over $S$ and an invertible sheaf $L$ on $P$ such that every geometric fiber is a polarized stable semiabelic variety over $\kappa(\bar{s})$.

Definition 1.1.15. A stable semiabelic pair over a scheme $S$ is a stable semiabelic scheme $G \curvearrowright P$ over $S$ and an effective relative Cartier divisor $\Theta$ on $P$ such that every geometric fiber is a stable semiabelic pair over $\kappa(\bar{s})$.

Once again, there are special cases of these definitions: for abelic, toric, stable toric, semiabelic schemes and pairs.

\section{Complexes of cones and lattice polytopes.}

Setup 1.1.16. We fix

1. a lattice $X \simeq \mathbb{Z}^{r}$,

2. a sublattice $i: Y \subset X$,

3. a trivial $X$-torsor $\bar{X}$.

The difference between $X$ and $\bar{X}$ is that in the latter we do not fix the origin.

The symbol $\mathbb{X}$ will denote the lattice $\mathbb{Z} \oplus X \simeq \mathbb{Z}^{r+1}$. We will denote by $X_{\mathbb{R}}\left(\operatorname{resp} . \bar{X}_{\mathbb{R}}, \mathbb{X}_{\mathbb{R}}\right)$ the real vector space $X \otimes \mathbb{R}($ resp. $\bar{X} \otimes \mathbb{R}, \mathbb{X} \otimes \mathbb{R})$.

Definition 1.1.17. A complex $\Omega$ of cones referenced by $X$ is a set $|\Omega|$ and a collection of distinct subsets $\{\omega\}$ called cones with $|\Omega|=\cup \omega$, together with a function $\rho:|\Omega| \rightarrow X_{\mathbb{R}}$ which identifies each $\omega$ with a finitely generated rational 
polyhedral cone in $X_{\mathbb{R}}$ with a vertex at the origin. The complex is supposed to be face-fitting: for any $\omega_{1}, \omega_{2} \in \Omega$ all faces of $\omega_{i}$ are in $\Omega$ and $\omega_{1} \cap \omega_{2}$ is a union of faces in $\Omega$. The set $|\Omega|$ comes with a (weak) classical topology: a subset is open if its intersections with all $\omega$ 's are open.

The empty set is not in $\Omega$ by definition. The set $\Omega$ has a natural partial order - by the reverse of inclusion of cones (so, if $\omega_{1}$ is a face of $\omega_{2}$, we say that $\omega_{1}$ is greater) and we will use this partial order. We will use the abbreviation poset for a partially ordered set.

Complexes of cones referenced by $\mathbb{X}$ are defined analogously.

Definition 1.1.18. A complex $\Delta$ of lattice polytopes referenced by $X$ is a set $|\Delta|$ and a collection of distinct subsets $\delta$ called cells with $|\Delta|=\cup \delta$, together with a function $\rho:|\Delta| \rightarrow X_{\mathbb{R}}$ which identifies each $\delta$ with a polytope in $X_{\mathbb{R}}$ with vertices in $X$. The complex is supposed to be face fitting: for any $\delta_{1}, \delta_{2} \in \Delta$ all faces of $\delta_{i}$ are in $\Delta$ and $\delta_{1} \cap \delta_{2}$ is a union of polytopes in $\Delta$. Once again, we will use the partial order on $\Delta$ by reverse of inclusion. We will include $\emptyset$ in the poset of polytopes by definition.

A complex of lattice polytopes referenced by $\bar{X}$ is defined in the same way, replacing $X_{\mathbb{R}}$ by $\bar{X}_{\mathbb{R}}$ and $X$ by $\bar{X}$.

Assumption 1.1.19. We will generally assume that $|\Delta|$ is connected and locally finite.

Remark 1.1.20. Note that the function $\rho$ need not be injective. The images of different cones or polytopes are allowed to intersect arbitrarily.

Definition 1.1 .21 . Let $\widetilde{\Delta}$ be a not necessarily connected and necessarily not finite complex of polytopes referenced by $X$ with a fixed action $X \curvearrowright \widetilde{\Delta}$ compatible with the translation action of $X$ on itself. Then, for each cell $\widetilde{\delta}$ and each $x \in X$ we have another cell $T_{x} \widetilde{\delta}$ and $T_{x} \rho(\widetilde{\delta})=\rho\left(T_{x} \widetilde{\delta}\right)$.

In this situation we will define a complex $\Delta=\widetilde{\Delta} / X$. Every cell $\delta$ in this complex is a polytope $\widetilde{\delta}$ with some of its faces glued according to the equivalence relation given by $X$-translations. Obviously, $X$ acts on the set $|\widetilde{\Delta}|$ properly discontinuously in the classical topology, and we have a set $|\widetilde{\Delta}| / X=|\Delta|=\cup \delta$.

Assuming as before that $|\Delta|$ is connected, let $\Delta^{\dagger}$ be one of the connected components of $\widetilde{\Delta}$ and denote by $Y$ the subgroup of $X$ leaving $\Delta^{\dagger}$ invariant. Then $\Delta=\Delta^{\dagger} / Y$. Note that the complex $\Delta^{\dagger}$ is referenced by $X$ and that it usually cannot be chosen canonically: the set of connected components of $\widetilde{\Delta}$ is in a 1-to-1 correspondence with $X / Y$.

We will call the complex $\Delta$ defined in the above described way a complex of lattice polytopes referenced by $\bar{X} / Y$. Note that the map $\rho:|\widetilde{\Delta}| \rightarrow X_{\mathbb{R}}$ induces a map $\rho:|\Delta| \rightarrow \bar{X}_{\mathbb{R}} / Y$. 
To deal with stable semiabelic varieties with nontrivial abelian part in a uniform way we will need yet another generalization of the above concepts that allows cells to be noncompact.

Definition 1.1.22. Assume:

1. a surjective homomorphism $X \rightarrow X^{\prime}$ to another lattice $X^{\prime}$ with kernel $X_{1}$;

2. a finite and connected complex of lattice polytopes $\Delta^{\prime}=\widetilde{\Delta}^{\prime} / X^{\prime}=\Delta^{\dagger} / Y^{\prime}$ referenced by $\bar{X}_{\mathbb{R}}^{\prime} / Y^{\prime}$.

Consider a complex $\widetilde{\Delta}$ which is the union of several copies of the pullback of the complex $\widetilde{\Delta}^{\prime}$ under $X \rightarrow X^{\prime}$. If $X_{1}$ is infinite then cells $\widetilde{\delta}$, pullbacks of cells $\widetilde{\delta}^{\prime}$, are not compact anymore. Assume in addition that we are given an action of $X$ on $\widetilde{\Delta}$ compatible with translation and extending the $X^{\prime}$-action on $\widetilde{\Delta}^{\prime}$ such that the cell complex $\widetilde{\Delta} / X$ is connected. Then the stabilizer group $Y_{1}$ of every cell $\widetilde{\delta}$ is a subgroup of finite index in $X_{1}$. This complex $\Delta=\widetilde{\Delta} / X$ is our most general cell complex. It has compact cells which are quotients by $Y_{1}$ of pullbacks of polytopes. There exists a split exact sequence $0 \rightarrow Y_{1} \rightarrow Y \rightarrow Y^{\prime} \rightarrow 0$ with a sublattice $Y \subset X$, and $\Delta=\Delta^{\dagger} / Y$ is referenced by $\bar{X} / Y$. Here, $\Delta^{\dagger}$ is one of the copies of the pullback of $\Delta^{\prime \dagger}$.

Example 1.1.23. If $X^{\prime}=0$ then $\Delta$ consists of one big cell $\bar{X}_{\mathbb{R}} / Y \simeq \mathbb{R}^{r} / \mathbb{Z}^{r}$.

Remark 1.1.24. A cell complex referenced by $\bar{X} / Y$ is a category which is essentially a poset. This means that for every $\delta_{1} \geq \delta_{2}$ there may be several arrows $\delta_{1} \rightarrow \delta_{2}$ but only the arrows $\delta \rightarrow \delta$ are identities.

Definition 1.1.25. For a connected complex $\Delta^{\dagger}$ covering $\Delta$ there is a unique connected complex $\Delta^{\ddagger}$ covering $\Delta^{\dagger}$ such that $\left|\Delta^{\ddagger}\right|$ is simply connected. Note that it is referenced by $X$ again and that $|\Delta|$ is the quotient of $\left|\Delta^{\ddagger}\right|$ by a free and discontinuous action of $\pi_{1}(\Delta)$.

Definition 1.1.26. We are in the

1. finite case if $Y=0$,

2. infinite periodic case if $Y$ has finite index in $X$,

3. intermediate case otherwise.

Definition 1.1.27. A pointed cell complex $(\Delta, C)$ is defined by adding a set $C \subset|\Delta| \cap \rho^{-1}(\bar{X} / Y)$ and subsets $C_{\delta}=C \cap \delta$ for each cell $\delta$ which must contain all the vertices of $\delta$. In the case of the pull-back from a lower-dimensional polytopal complex $\widetilde{\Delta}^{\prime}$, the images of $C_{\delta}$ must contain the vertices of $\widetilde{\delta}^{\prime}$. 
Definition 1.1.28 (order on complexes). Let $\mathcal{D}_{1}, \mathcal{D}_{2}$ be two cone or cell decompositions, or pointed cell decompositions referenced by $X_{\mathbb{R}}$. We say that $\mathcal{D}_{1}$ is a sub-decomposition of $\mathcal{D}_{2}$ and denote it $\mathcal{D}_{1} \leq \mathcal{D}_{2}$ if the cones, resp. cells, resp. pointed cells of $\mathcal{D}_{1}$ form a subdivision of those of $\mathcal{D}_{2}$. For pointed cells, $C_{1}$ has to be a subset of $C_{2}$.

Definition 1.1.29. We will call a complex $\mathcal{D} 1$-sheeted convex if $\rho$ is injective and $\rho(|\mathcal{D}|)$ is convex. Explicitly, this means that

1. in the case of cones, $\Omega$ is a subdivision of a single finitely generated rational cone;

2. in the case of cells, $\left|\Delta^{\dagger}\right|$ is a pullback of a single polytope of lower dimension.

The following definition is consistent with the one for simplicial complexes appearing in connection with Stanley-Reisner rings (see [Sta1], [BH]), as well as with the more general definition of [Bac] for arbitrary posets. In [Yuz] CM posets were called $k$-spherical.

Definition 1.1.30. A complex $\mathcal{D}$ is called almost Cohen-Macaulay with respect to $k$, abbreviated ACM, or locally Cohen-Macaulay if it is locally finite and for every point $u \in|\mathcal{D}|$ one has $\widetilde{H}_{i}(|\mathcal{D}|,|\mathcal{D}|-u, k)=0$ for $0 \leq i<\operatorname{dim} \mathcal{D}$. Here $\widetilde{H}_{i}$ denotes the reduced singular homology. It is called Cohen-Macaulay with respect to $k$ if, in addition $\widetilde{H}_{i}(|\mathcal{D}|, k)=0$ for $0 \leq i<\operatorname{dim} \mathcal{D}$.

Example 1.1.31. One has the following implications: $\mathcal{D}$ is 1 -sheeted convex $\Rightarrow|\mathcal{D}|$ is a topological manifold (possibly with boundary) $\Rightarrow \mathcal{D}$ is locally CM.

\subsection{Main results.}

A. Moduli problem: abelic case.

Lemma 1.2.1 (see 3.0.1). Every polarized abelic scheme $(A \curvearrowright P, L) / S$ of degree $d$ defines in a natural way a polarization $\lambda: A \rightarrow A^{t}$ of degree $d=\sqrt{\operatorname{deg} \lambda}$ on $A / S$.

TheOrem 1.2.2 (3.0.12). $\quad$ 1. The moduli stack $\mathcal{A P}_{g, d}$ of abelic pairs $(P, \Theta)$ of degree $d$ is a separated Artin stack with finite stabilizers and it comes with a natural map of relative dimension $d-1$ to the stack $\mathcal{A}_{g, d}$ of polarized abelian varieties.

2. $\mathcal{A P}_{g, d}$ has a coarse moduli space $\mathrm{AP}_{g, d}$ which is a separated scheme and comes with a natural projective map of relative dimension $d-1$ to $\mathrm{A}_{g, d}$.

3. Over $\mathbb{Z}[1 / d]$ the schemes $\mathrm{AP}_{g, d}$ and $\mathrm{A}_{g, d}$ are disjoint unions of components naturally labeled by g-dimensional 1-cell complexes $\bar{X}_{\mathbb{R}} / Y$ with $|X / Y|=d$. 
THEOREM 1.2.3 (3.0.7, 3.0.9). In the principally polarized case, i.e. when $d=1, \mathcal{A P}_{g}=\mathcal{A}_{g}$ and $\mathrm{AP}_{g}=\mathrm{A}_{\mathrm{g}}$.

B. Classification of stable toric varieties and pairs over closed fields. We start with the toric case because it is easier to formulate.

Setup 1.2.4. We fix a torus $T$ of dimension $r$. Varieties in this subsection all have $T$-action. We denote by $X \simeq \mathbb{Z}^{r}$ the Cartier dual of $T$, so that $T=\operatorname{Hom}\left(X, \mathbb{G}_{m}\right)$.

Symbols $\underline{T}, \underline{\mathbb{T}}$, etc. denote certain constructible sheaves of commutative groups on the topological space $|\mathcal{D}|$ to be defined in the main text. The reader can guess their meanings from the notation. The complex $\widehat{\mathbb{M}}^{*}$ is defined as the cylinder of the morphism of cochain complexes $C^{*}(\underline{\mathbb{T}}) \rightarrow C^{*}\left(\widehat{\underline{\operatorname{Fun}}}_{C}\right)$.

TheOREm 1.2.5 $(2.3 .14,2.3 .18)$. There is a 1-to-1 correspondence between

1. affine stable toric varieties $P$,

2. the following data:

(a) a finite complex $\Omega$ of cones referenced by $X$,

(b) an element of the cohomology group $H^{1}(\Omega, \underline{T}) / \operatorname{Sym} \Omega$, where $\operatorname{Sym} \Omega$ is the (finite) group of symmetries of $\Omega$ compatible with automorphisms of $X$.

Moreover, Aut $P$ is a finite extension of $H^{0}(\Omega, \underline{T})$.

TheOREM 1.2.6 $(2.4 .7,2.4 .11,5.3 .8)$. There is a 1-to-1 correspondence between

1. polarized stable toric varieties $(P, L)$,

2. the following data:

(a) a sublattice $Y \subset X$ and an $X$-torsor $\bar{X}$,

(b) a finite complex $\Delta$ of lattice polytopes referenced by $\bar{X} / Y$,

(c) an element of $H^{1}(\Delta, \mathbb{T}) / \operatorname{Sym} \Delta$.

Moreover, $\operatorname{Aut}(P, L)$ is a finite extension of $H^{0}(\Omega, \mathbb{T})$.

TheOREM 1.2.7 $(2.6 .8,5.4 .3)$. There is a 1-to-1 correspondence between

1. stable toric pairs $(P, \Theta)$,

2. the following data: 
(a) a sublattice $Y \subset X$ and an $X$-torsor $\bar{X}$,

(b) a finite complex $(\Delta, C)$ of pointed lattice polytopes referenced by $\bar{X} / Y$,

(c) an element of the cohomology group $H^{1}\left(\widehat{\mathbb{M}}^{*}\right) / \operatorname{Sym} \Delta$.

Moreover, $\operatorname{Aut}(P, \Theta)$ is a finite extension of $H^{0}\left(\widehat{\mathbb{M}}^{*}\right)$.

C. Classification of SSAVs and pairs over closed fields.

Setup 1.2.8. We fix a semiabelian variety $G$ with the abelian part $A$ of dimension $a$ and the toric part $T$ of dimension $r, a+r=g$. Varieties in this subsection all have a $G$-action. As before, we denote by $X^{\prime} \simeq \mathbb{Z}^{r}$ the Cartier dual of $T$. The extension $G$ is defined by a homomorphism $c: X^{\prime} \rightarrow A^{t}$.

We also fix a cell complex referenced by $\bar{X}^{\prime} / Y^{\prime}$. We will denote by $i$ : $\pi_{1}\left(\left|\Delta^{\prime}\right|\right) \rightarrow X^{\prime}$ the natural projection.

Additionally, one has to have

1. a polarization $\lambda: A \rightarrow A^{t}$,

2. a homomorphism $c^{t}: \pi_{1}\left(\left|\Delta^{\prime}\right|\right) \rightarrow A$ such that $\lambda \circ c^{t}=c \circ i: \pi_{1}\left(\left|\Delta^{\prime}\right|\right) \rightarrow A^{t}$.

The first step is to classify the polarized varieties $(P, L)$ and pairs $(P, \Theta)$ with additional data:

1. a fixed point in one of the minimal-dimensional orbits on $P$, which is isomorphic to $A$,

2. an ample line bundle $\mathcal{M}$ on $A$ such that $\lambda(\mathcal{M})=\lambda$.

With these additional data, the theorems of the previous section remain literally true if one understands $H^{p}$ as functions on the homology groups $H_{p}$ with the values in certain $\mathbb{G}_{m}(k)$-torsors instead of $\mathbb{G}_{m}(k)$; see 5.3.8, 5.4.3.

In general, the isomorphisms are obtained by dividing the family by the equivalence relation corresponding to forgetting the choice of the point in a minimal orbit.

Hence, every polarized stable semiabelic variety (resp. pair) is labeled by a complex $\Delta^{\prime}$ (resp. pointed complex) of lattice polytopes for the toric part. In addition, if chark does not divide $d=\operatorname{deg} \lambda(\mathcal{M})$ then one naturally associates to $P$ or $(P, \Theta)$ a complex $\Delta$ of dimension $\operatorname{dim} \Delta^{\prime}+a$ with nonpolytopal cells if $a>0$.

D. Classification of SSAVs and pairs over $\mathbb{C}$. Over $\mathbb{C}$, a semiabelian variety $G$ can be written as $\left(\mathbb{C}^{*}\right)^{g} / Y=T / Y$ and a variety $P$ with $G$-action in this way can be considered to be "toric". A different choice of such a representation corresponds to a different choice of an isotropic sublattice in an appropriate symplectic lattice. 
TheOREM 1.2.9 (5.5.6). Polarized stable semiabelic varieties (resp. pairs) with a fixed T-action are parametrized by a cell complex $\Delta$ and an open subset in $H^{1}(\Delta, \underline{\widehat{\mathbb{X}}})\left(\right.$ resp. $\left.H^{1}\left((\Delta, C), \widehat{\mathbb{M}}^{*}\right)\right)$, consisting of classes satisfying the Riemann positivity condition, modulo the group of symmetries of $\Delta$.

TheOREM 1.2.10 (2.7.2, 5.5.7). A stable semiabelic pair with a fixed $T$-action naturally defines a moment map Mom $: P(\mathbb{C}) \rightarrow|\Delta|$. A fiber over a point in the interior of a $k$-dimensional cell is isomorphic to $\left(S^{1}\right)^{k}=U(1)^{k}$.

E. Cohomologies of polarized stable semiabelic varieties.

Theorem 1.2.11 (2.5.1, 5.4.1). For all $p, d>0, H^{p}\left(P, L^{d}\right)=0$.

F. Types and singularities of SSAVs. We will call the complex $\Omega$, resp. $\Delta$, associated to an affine, resp. polarized, SSAV $P$ by the classification theorems above the type of $P$.

Example 1.2.12. 1) Finite case. The numerical type of a polarized toric variety is a lattice polytope $Q \subset \bar{X}_{\mathbb{R}} \simeq \mathbb{R}^{r}$.

2) Infinite periodic case. Let $(A \curvearrowright P, L)$ be a polarized abelic variety of degree $d$, chark $\nmid d$. Let $\lambda: A \rightarrow A^{t}$ be the induced polarization on $A$ with $K(\lambda) \simeq H \times \widehat{H}$, and say $H=X / Y$. In this case the numerical type of $(P, L)$ is $\bar{X}_{\mathbb{R}} / Y \simeq \mathbb{R}^{g} / \mathbb{Z}^{g}$.

TheOREM 1.2.13 (2.10.1, 5.7.4). Let $(G \curvearrowright P, L)$ be a polarized stable semiabelic scheme over a connected scheme $S$. If for two geometric fibers the types are defined (i.e. chark does not divide the degree $d$ of polarization on the abelian part) then $\left|\Delta_{1}\right|$ and $\left|\Delta_{2}\right|$ can be noncanonically identified.

In this paper we will only consider the moduli problem for stable semiabelic pairs that have the types 1 ) or 2 ) of the above example or their subdivisions.

Theorem 1.2.14 $(2.3 .19,2.4 .12,5.3 .9)$. In the affine case, $\Omega$ is $\mathrm{CM} \Rightarrow P$ is $\mathrm{CM}$. In the polarized case, $\Delta$ is locally $\mathrm{CM} \Rightarrow P$ is $\mathrm{CM}$.

G. Moduli problem: the finite case. In the finite case we can make the situation more restricted by fixing $T, X$ once and for all and requiring that all automorphisms of stable toric pairs preserve them. We then have:

Theorem 1.2.15 (2.10.10, 2.11.11, 2.12.3, 2.12.13). For each lattice polytope $Q$, over $\mathbb{Z}$ or a field:

1. The moduli stack of stable toric pairs $(P, \Theta)$ of type $\leq Q$ is a proper Artin stack with finite stabilizers.

2. It has a coarse moduli space $\mathrm{M}_{\mathrm{Q}}$. 
3. $\mathrm{M}_{\mathrm{Q}}$ is projective.

4. $\mathrm{M}_{\mathrm{Q}}$ is naturally stratified, and every stratum corresponds in a 1-to-1 way to a cell decomposition of $Q$ (resp. a pointed cell decomposition of $(Q, C)$, where $C=Q \cap X)$.

5. The normalization of every irreducible component of $\mathrm{M}_{\mathrm{Q}}$ is a projective toric scheme corresponding to a generalized secondary polytope.

6. The toric scheme $M_{\Sigma(Q)}$ for the secondary polytope of $Q$ is isomorphic to the main irreducible component of $\left(M_{Q}\right)_{\mathrm{red}}$.

H. Moduli problem: the infinite periodic case.

Theorem 1.2.16 (5.10.1). 1. The component $\overline{\mathcal{A P}}_{g, d}$ of the moduli stack of semiabelic pairs containing $\mathcal{A P}_{g, d}$ and pairs of the same numerical type is a proper Artin stack with finite stabilizers;

2. It has a coarse moduli space $\overline{\mathrm{AP}}_{g, d}$ as a proper algebraic space;

3. The space $\overline{\mathrm{AP}}_{g, d}$ is naturally stratified according to the toric part of $(P, \Theta)$, and every stratum corresponds 1-to-1 to a cell decomposition of $\bar{X}_{\mathbb{R}}^{\prime} / Y^{\prime}$ with $\operatorname{dim} X^{\prime} \leq g$ and $\left|X^{\prime} / Y^{\prime}\right|$ dividing $d$, modulo symmetries.

4. Over $\mathbb{Z}[1 / d]$ the space $\overline{\mathrm{AP}}_{g, d}$ has a nicer stratification, with every stratum corresponding 1-to-1 to a cell decomposition of $\bar{X}_{\mathbb{R}} / Y$ with $\operatorname{dim} X=g$ and $|X / Y|=d$, modulo symmetries.

THEOREM 1.2.17 (5.11.6). In the principally polarized case (when $Y=X)$ the toroidal compactification of $A_{g}$ for the second Voronoi decomposition is isomorphic to the main irreducible component of $\overline{\mathrm{AP}}_{g}$, the one containing $\mathrm{A}_{g}=\mathrm{AP}_{g}$.

Theorem 1.2.18 (5.12.8). The toroidal compactification of $\mathrm{A}_{g}$ for the second Voronoi decomposition is projective.

\section{Stable toric varieties and pairs}

2.1. Complexes, posets and (co)sheaves on them. Let $I$ be a poset, i.e. a partially ordered set. We can look at it as being a category associating an arrow $i_{1} \rightarrow i_{2}$ to each pair $i_{1} \geq i_{2}$. (Alternatively, we can associate to such a pair an arrow $i_{2} \rightarrow i_{1}$. The choice is entirely a matter of taste. Ours will be more convenient for working with cones and polytopes.) 
Definition 2.1.1. The order topology on $I$, which we will denote $I_{\text {ord }}$, is the topology in which the open sets are the increasing sets: $i \in U$ and $j \geq i \Rightarrow j \in U$. The complements of the increasing sets are the decreasing sets, so these are closed. The open cover $\mathcal{U}_{\text {std }}$ by the sets $U_{\geq i}=\{j \geq i\}$ refines any other open cover.

Although the major part of the results below requires very little of the poset $I$, all our posets will satisfy the following:

Assumption 2.1.2. There is a global bound on the length of all chains in $I$.

Assumption 2.1.3. $I$ is locally finite; i.e., for each $i$ there are only finitely many $j$ comparable to it.

Under Assumption 2.1.2, $I$ is covered by the sets $U_{\geq i_{0}}$ for all minimal $i_{0}$. We will denote this locally finite open cover $\left\{U_{\geq i_{0}} \mid i_{0} \in I_{\min }\right\}$ by $\mathcal{U}_{\min }$.

Definition 2.1.4. A sheaf $F$ of sets on $I_{\text {ord }}$ is the same as an inverse system of sets $F_{i}$, called stalks, indexed by $I$. Hence, for every pair $i_{1} \geq i_{2}$ we are given a map $\varphi_{i_{2} i_{1}}: F_{i_{2}} \rightarrow F_{i_{1}}, \varphi_{i i}=\mathrm{id}$ and $\varphi_{i_{2} i_{1}} \circ \varphi_{i_{3} i_{2}}=\varphi_{i_{3} i_{1}}$. For any subset $J \subset I$, one has the set of sections

$$
F(J)=\varliminf_{J} F_{j}=\left\{\left(f_{j}\right) \in \prod_{J} F_{j} \mid \varphi_{j_{2} j_{1}}\left(f_{j_{2}}\right)=f_{j_{1}}\right\} .
$$

We have obvious restriction maps $F\left(J_{2}\right) \rightarrow F\left(J_{1}\right)$ for all $J_{1} \subset J_{2}$, and a presheaf defined this way is automatically a sheaf.

Dually, a cosheaf $G$ of sets on $I_{\text {ord }}$ is the same as a direct system $\left\{G_{i}, \psi_{i_{1} i_{2}}\right.$ : $\left.G_{i_{1}} \rightarrow G_{i_{2}}\right\}$ of sets. For any subset $J \subset I$,

$$
G(J)=\lim _{J} G_{j}=\coprod_{J} G_{j} /\left(g_{j_{1}} \sim \psi_{j_{1} j_{2}}\left(g_{j_{1}}\right)\right) .
$$

If the $F_{i}$ 's have an extra structure, i.e. they are groups, semigroups or algebras, and $\varphi$ 's preserve it, the inverse limit has the same structure, so one has sheaves of groups etc. The situation with the cosheaves is a bit more complicated. The direct limits in the categories of commutative groups and semigroups exist but as sets they do not coincide with the ones above. Instead, they are given by the formula

$$
G(J)=\underline{\lim }_{J} G_{j}=\bigoplus_{J} G_{j} /\left\langle g_{j_{1}} \sim \psi_{j_{1} j_{2}}\left(g_{j_{1}}\right)\right\rangle .
$$

So, we have to be a little careful about the category we work in. This will always be clear from the context.

When working with the (co)sheaves of commutative groups - and this will be the default case - we can apply the standard machinery of sheaf cohomologies [God] and cosheaf homologies (see [Bre]). By the very definition, we 
have

$$
H^{0}\left(I_{\text {ord }}, F\right)=\lim _{I} F_{i}, \quad H_{0}\left(I_{\text {ord }}, G\right)=\underline{\lim }_{I} G_{i} .
$$

Since the open cover $\mathcal{U}_{\text {std }}$ refines any other, the Cech cohomologies $\breve{H}^{q}\left(I_{\text {ord }}, F\right)$ are isomorphic to $\breve{H}^{q}\left(\mathcal{U}_{\text {std }}, F\right)$. The natural homomorphisms $\breve{H}^{q}\left(I_{\text {ord }}, F\right) \rightarrow H^{q}\left(I_{\text {ord }}, F\right)$ are isomorphisms for $q=0,1$ and are injective for $q \geq 2$ (since $I_{\text {ord }}$ is not paracompact, they are not guaranteed to be isomorphisms in general). Dually, the natural homomorphisms $H_{q}\left(I_{\text {ord }}, F\right) \rightarrow$ $\breve{H}_{q}\left(I_{\text {ord }}, F\right)$ are isomorphisms for $q=0,1$ and are surjective for $q \geq 2$.

The following case will be of special interest:

Definition 2.1.5. The poset $I$ is called a join prelattice or a join semilattice if any two elements $i_{1}, i_{2}$ have the least upper bound $\operatorname{lub}\left(i_{1}, i_{2}\right)$. In this case for all $i_{1}, i_{2} \in I$ the set $U_{\geq i_{1}} \cap U_{\geq i_{2}}$ equals $U_{\geq \operatorname{lub}\left(i_{1}, i_{2}\right)}$.

Lemma 2.1.6. 1. Any sheaf $F$ (resp. cosheaf) on the open set $U_{\geq i}$ is flabby and hence acyclic.

2. If $I$ is a join prelattice then $H^{q}\left(I_{\text {ord }}, F\right)=H^{q}\left(\mathcal{U}_{\text {min }}, F\right)$ (resp. for a cosheaf).

Proof. We will prove these statements for a sheaf. For a cosheaf use a dual argument. 1) is obvious since any open subset $U$ of $U_{\geq i}$ contains $i$ and so $\Gamma(U, F)=F_{i}$. Part 2) follows because $F$ is acyclic when restricted to any of $\cap U_{\geq i}$ (see [God, 5.9.2]).

The order topology is quite convenient for computations because there are just so few open sets. However, it is not very intuitive. Another drawback is that a free action by a group such as $\mathbb{Z} / n \mathbb{Z}$ or $\mathbb{Z}^{r}$ on $I_{\text {ord }}$ is often not discontinuous. There is a standard construction associating to each poset $I$ (and, in fact, to any category) an ordinary simplicial complex $B I$ which comes with the (weak) classical topology: a subset is open if and only if its intersection with each simplex is open.

Definition 2.1.7. $B I$ is a simplicial complex which has a simplex $\sigma_{i_{0} \ldots i_{q}}$ for each strictly increasing chain $i_{0}<\ldots<i_{q}$ in $I$, with obvious inclusions.

Clearly, $B I$ is a disjoint union of the interiors $\sigma_{i_{0} \ldots i_{q}}^{0}$ of the simplices.

Definition 2.1.8. Define two maps

$$
\begin{aligned}
\phi_{l}: B I \rightarrow I, & \sigma_{i_{0} \ldots i_{q}}^{0} \mapsto i_{0} & & \text { mapping to the least } i, \\
\phi_{g}: B I \rightarrow I, & \sigma_{i_{0} \ldots i_{q}}^{0} \mapsto i_{q} & & \text { mapping to the greatest } i .
\end{aligned}
$$


Denote

$$
\begin{aligned}
U_{i} & =\phi_{g}^{-1}\left(U_{\geq i}\right), & W_{i} & =\phi_{g}^{-1}(i), \\
V_{i} & =\phi_{l}^{-1}\left(U_{\geq i}\right), & V_{i}^{0} & =\phi_{l}^{-1}(i) .
\end{aligned}
$$

Lemma 2.1.9. Let $U$ be an open subset of $I_{\text {ord }}$. Then

1. $\phi_{g}^{-1}(U)$ is open, i.e., $\phi_{g}$ is continuous. In particular, $U_{i}$ are open and $\bar{W}_{i}=\phi_{g}^{-1}\left(U_{\leq i}\right)$ are closed.

2. $\phi_{l}^{-1}(U)$ is closed, in particular $V_{i}$ are closed.

3. $W_{i}=U_{i} \cap \bar{W}_{i}$ and $V_{i}^{0}$ are locally closed and contractible to the point $\sigma_{i}=W_{i} \cap V_{i}^{0}$.

Proof. This requires only a routine check.

Since $\phi_{g}$ is continuous, we can consider the pullback $\phi_{g}^{-1} F$. This is a constructible sheaf on $B I$ whose restriction to each $W_{i}$ is a constant sheaf $F_{i}$ and the stalk at a point $x \in W_{i}$ is $F_{i}$. Analogously, $\phi_{g}^{-1} G$ is a constructible cosheaf whose restriction on each $W_{i}$ is a constant cosheaf $G_{i}$.

Lemma 2.1.10. For all $q, H^{q}\left(B I, \phi_{g}^{-1} F\right)=H^{q}\left(I_{\text {ord }}, F\right)$ and $H_{q}\left(B I, \phi_{g}^{-1} G\right)=H_{q}\left(I_{\text {ord }}, G\right)$.

Proof. For a sheaf $F$ the statement is an application of the Vietoris mapping theorem. Indeed, each fiber $W_{i}$ is taut in $B I$ since the latter is a $\mathrm{CW}$ complex, and its higher cohomologies with the coefficients in $F_{i}$ vanish because it is contractible. The statement for a cosheaf $G$ is obtained by a dual argument.

From now we will write $H^{q}(I, F)$ or simply $H^{q}(F)$ if the poset $I$ is understood $\left(\operatorname{resp.} H_{q}(I, G), H_{q}(G)\right)$.

Definition 2.1.11 (Dual sheaf to a cosheaf of semigroups). Let $G$ be a cosheaf of commutative semigroups on $I_{\text {ord }}$, which we now can also consider as a cosheaf on its geometric realization $B I$. The system $G_{i}$ defines a cosheaf of semigroup algebras $\mathbb{Z}\left[G_{i}\right]$ and, dually, an inverse system of affine semigroup schemes Spec $\mathbb{Z}\left[G_{i}\right]$. For any scheme $S$, we obtain a sheaf of commutative semigroups for the multiplication which we will denote by $\widehat{G}(S)$ :

$$
\begin{aligned}
\widehat{G}(S)_{i} & =\operatorname{Spec} \mathbb{Z}\left[G_{i}\right](S)=\left\{\text { morphisms } S \rightarrow \operatorname{Spec} \mathbb{Z}\left[G_{i}\right]\right\} \\
& =\left\{\text { semigroup homomorphisms } G_{i} \rightarrow \Gamma\left(S, \mathcal{O}_{S}\right)\right\} .
\end{aligned}
$$

If the semigroups $G_{i}$ are groups then 


$$
\widehat{G}(S)_{i}=\left\{\text { group homomorphisms } G_{i} \rightarrow \mathbb{G}_{m}(S)=\Gamma\left(S, \mathcal{O}_{S}\right)^{*}\right\}
$$

and $\widehat{G}(S)$ is a sheaf of commutative groups. The cosheaf $G$ here has an "absolute" character and the dual cosheaf is relative to the choice of a scheme $S$.

LEMMA 2.1.12. 1. If the $G_{i}$ are free abelian groups then there is the following noncanonically split exact sequence:

$$
0 \rightarrow \operatorname{Ext}\left(\breve{H}_{p-1}(G), \mathbb{G}_{m}(S)\right) \rightarrow \breve{H}^{p}(\widehat{G}(S)) \rightarrow \operatorname{Hom}\left(\breve{H}_{p}(G), \mathbb{G}_{m}(S)\right) \rightarrow 0 .
$$

2. Without any assumptions on $G_{i}$, when $S=\operatorname{Spec} k$ is the spectrum of an algebraically closed field, $\breve{H}^{p}(\widehat{G}(S))=\operatorname{Hom}\left(\breve{H}_{p}(G), \mathbb{G}_{m}(k)\right)$.

Proof. Both parts are immediate applications of the universal coefficient theorem (e.g. [Spa, Thm.5.5.3]) and the fact that for an algebraically closed field, $\mathbb{G}_{m}(k)=k^{*}$ is a divisible group, hence an injective $\mathbb{Z}$-module.

2.1.13. Now consider a complex of lattice polytopes $\Delta=\left\{\delta_{i} ; i \in I\right\}$ referenced by a lattice $X$ with the partial order on $I$ which is the opposite to the order by inclusion. Sometimes, we will write $\Delta$ instead of $I$. Clearly, our assumptions on the posets are satisfied. Pick a point $p_{i}$ in the interior of each polytope $\delta_{i}$ and identify the simplex $\sigma_{i_{0} \ldots i_{q}}$ with a subset of $\delta_{i_{q}}$ with vertices $p_{i_{0}} \ldots p_{i_{q}}$. Then $B I$ is nothing but the barycentric decomposition of $\Delta$. In particular, $B I=|\Delta|$. The locally closed sets $V_{i}^{0}$ are interiors $\delta_{i}^{0}$ of the polytopes $\delta_{i}$, and the closed sets $V_{i}$ are the polytopes $\delta_{i}$ themselves. For a minimal by reverse inclusion polytope $\delta_{i}$ the set $W_{i}$ is the barycenter of $\delta_{i}$.

Next, consider a complex of cones $\Omega=\left\{\omega_{i} ; i \in I\right\}$ referenced by a lattice $X$. Again, we order the index set $I$ by the order opposite to the inclusion of cones. The relationship between $|\Omega|$ and $B I=B \Omega$ in this case is slightly more complicated. Assume that $|\Omega|$ is connected. Since $\Omega$ is face-fitting, the minimal faces of all $\omega$ 's are equal to a linear subspace $\omega_{\min } \subset X_{\mathbb{R}}$. If we denote $X_{\mathbb{R}}^{\prime}=X_{\mathbb{R}} / \omega_{\min }$ then every $\omega_{i}$ is the preimage of a strictly convex cone $\omega_{i}^{\prime}$ in $X_{\mathbb{R}}^{\prime}$.

Definition 2.1.14. Fix an arbitrary norm on $X_{\mathbb{R}}^{\prime}$ and let $S^{r^{\prime}-1}$ be the unit sphere centered at the origin. The spherical complex $S \Omega=S \Omega^{\prime}$ induced by $\Omega$ consists of the sets $\omega_{i}^{\prime} \cap S^{r^{\prime}-1}$. It will include the empty set by definition.

2.1.15. Pick a point $p_{i}$ in the interior of each spherical cell $\omega_{i}^{\prime} \cap S^{r^{\prime}-1}$ and, in addition, a point $p_{\text {min }}$ for the minimal cone. It is now clear that $B(I \backslash \min )$ is the barycentric decomposition of $|S \Omega|$ and that $B \Omega$ is the cone over it with the apex $p_{\min }$. 
Remark 2.1.16. It is clear that $|\Omega|$ and $|B \Omega|$ are homotopy equivalent and that the sheaf $\phi_{g}^{-1} F$ on $B \Omega$ has the same cohomologies as the natural constructible sheaf on $|\Omega|$.

Definition 2.1.17. For a complex $\Delta$ of polytopes in $X$ we define the complex Cone $\Delta$ as consisting of cones over all $\delta_{i} \subset\left(1, X_{\mathbb{R}}\right)$ in $\mathbb{X}_{\mathbb{R}}$, plus $\{0\}$.

Note that the posets Cone $\Delta$ and $\Delta$ (which includes $\emptyset$ ) are isomorphic, as well as are the posets $\Omega$ and $S \Omega$, and that the topological spaces $\mid S$ Cone $\Delta \mid$ and $|\Delta|$ are homeomorphic.

\subsection{Basic (co) sheaves and relations between them.}

Definition 2.2.1. The cosheaf $\underline{X}$ on $\Omega_{\text {ord }}$ is defined by associating to each cone $\omega_{i}$ the lattice $X_{i}=X \cap \mathbb{R} \omega_{i}$, the saturated sublattice of $X$ generated by $\omega_{i}$. If $i>j$, i.e. $\omega_{i}$ is a face of $\omega_{j}$, then $X_{i} \subset X_{j}$, so this defines a cosheaf. The dual sheaf $\widehat{X}=T$ is defined by the groups $\widehat{X}_{i}=T_{i}=\operatorname{Hom}\left(X_{i}, \mathbb{G}_{m}(S)\right)$.

Definition 2.2.2. Let $\Delta$ be a complex of lattice polytopes referenced by $X$. For every polytope $\delta_{i} \subset\left(1, X_{\mathbb{R}}\right)$ let $\mathbb{X}_{i}$ be the lattice $\mathbb{X} \cap \mathbb{R} \delta_{i}$. These groups define the cosheaf $\underline{\mathbb{X}}$. The groups $\underline{\mathbb{X}}_{i}=\underline{\mathbb{T}}_{i}=\operatorname{Hom}\left(\mathbb{X}_{i}, \mathbb{G}_{m}(S)\right)$ define the dual sheaf $\underline{\mathbb{X}}=\mathbb{T}$. Now, $\Delta_{\text {ord }} \simeq$ Cone $\Delta_{\text {ord }}$, and the cosheaf $\underline{\mathbb{X}}$ is the same as the cosheaf $\underline{X}$ on Cone $\Delta$ defined above.

Definition 2.2.3. For a pointed cell complex $(\Delta, C)$ we define a cosheaf $\underline{\text { Fun }}=\underline{\operatorname{Fun}}[\Delta, C]$ of commutative groups by setting $\underline{\operatorname{Fun}}_{i}=\operatorname{Fun}\left(C_{i}, \mathbb{Z}\right)$. and the dual sheaf $\underline{\underline{\text { Fun }}}=\widehat{\text { Fun }}[\Delta, C](S)$ by $\underline{\operatorname{Fun}}_{i}=\operatorname{Fun}\left(C_{i}, \mathbb{G}_{m}(S)\right)$. Further, we define a cosheaf and the dual sheaf of semigroups by setting $\underline{\text { Fun }}_{\geq 0, i}(S)=$ $\operatorname{Fun}\left(X \cap \delta_{i} \backslash C_{i}, \mathbb{Z}_{\geq 0}\right) \oplus \operatorname{Fun}\left(C_{i}, \mathbb{Z}\right)$ and $\underline{\operatorname{Fun}}_{\geq 0, i}(S)=\operatorname{Hom}\left(\operatorname{Fun}_{\geq 0, i}, \mathcal{O}_{S}(S)(S)\right)$. Also, define the cosheaves $\underline{\mathbb{L}}, \underline{\mathbb{K}}$ and the dual sheaves $\underline{\mathbb{L}}(S), \underline{\mathbb{K}}(S)$ by

$$
\begin{aligned}
& \underline{\mathbb{L}}_{i}=\operatorname{ker} \phi_{C_{i}}, \quad \underline{\mathbb{K}}_{i}=\text { coker } \phi_{C_{i}} \\
& \text { where } \phi_{C_{i}}: \operatorname{Fun}\left(C_{i}, \mathbb{Z}\right) \rightarrow \mathbb{X}_{i}, \quad\left(n_{x}\right) \mapsto \sum n_{x}(1, x) .
\end{aligned}
$$

All these (co)sheaves are relative to our choice of $(\Delta, C)$.

Note that since we assumed that $\operatorname{Conv} C_{i}=\delta_{i}$, the groups $\mathbb{K}_{i}$ are finite. We have the following exact sequence of cosheaves on $\Delta$ :

$$
0 \rightarrow \underline{\mathbb{L}} \rightarrow \underline{\text { Fun }} \stackrel{\phi}{\rightarrow} \underline{\mathbb{X}} \rightarrow \underline{\mathbb{K}} \rightarrow 0 .
$$

When $S=\operatorname{Spec} k, k=\bar{k}$, the dual sequence of sheaves is also exact:

$$
1 \rightarrow \underline{\widehat{\mathbb{K}}} \rightarrow \underline{\widehat{\mathbb{X}}} \stackrel{\hat{\phi}}{\rightarrow} \underline{\widehat{\mathrm{Fun}}} \rightarrow \underline{\widehat{\mathbb{L}}} \rightarrow 1 \text {. }
$$

Lemma 2.2.4. $H_{p}(\Delta, \underline{\text { Fun }})=0$ for $p>0$ and $H_{0}(\Delta, \underline{\text { Fun }})=\operatorname{Fun}(C, \mathbb{Z})$. Dually, $H^{p}(\Delta, \widehat{\underline{\text { Fun }}})=0$ for $p>0$ and $H^{0}(\Delta, \widehat{\widehat{\text { Fun }}})=\operatorname{Fun}\left(C, \mathbb{G}_{m}(S)\right)$. 
Proof. Indeed, the sheaf $\widehat{\widehat{\text { Fun }}}$ is a direct sum of sheaves $\widehat{\text { Fun }}_{c}, c \in C$, each of which is a constant sheaf on a contractible set $\bar{W}_{c}$.

Definition 2.2.5. We introduce the complex $\mathbb{M}_{*}=\mathbb{M}_{*}[\Delta, C]$ as the mapping cylinder of the induced homomorphism of Cech chain complexes $\phi$ : $C_{*}\left(\mathcal{U}_{\text {std }}, \underline{\text { Fun }}\right) \rightarrow C_{*}\left(\mathcal{U}_{\text {std }}, \underline{\mathbb{X}}\right)$, or, if the poset $\Delta$ is a join semilattice, of Cech chain complexes with respect to the cover $\mathcal{U}_{\text {min }}$. Explicitly, one has $\mathbb{M}_{p}=$ $C_{p-1}(\underline{\text { Fun }}) \oplus C_{p}(\underline{\mathbb{X}})$ with the differential $\left(d,(-1)^{p} \phi\right) \oplus d: \mathbb{M}_{p+1} \rightarrow \mathbb{M}_{p}$

Dually, we have a complex $\widehat{\mathbb{M}}^{*}=\widehat{\mathbb{M}}^{*}[\Delta, C]$ which is the cylinder of the map of Cech cochain complexes $\hat{\phi}: C^{*}\left(\mathcal{U}_{\text {std }}, \underline{\widehat{\mathbb{X}}}(S)\right) \rightarrow C^{*}\left(\mathcal{U}_{\text {std }}, \underline{\widehat{\operatorname{Fun}}}(S)\right)$. Now $\widehat{\mathbb{M}}^{p}(S)=\operatorname{Hom}\left(\mathbb{M}_{p}, \mathbb{G}_{m}(S)\right)$.

Definition 2.2.6. By adding to each of the complexes above one more group $-\operatorname{Fun}(C, \mathbb{Z}), \mathbb{X}$ etc., we obtain the augmented complexes. We will call their cohomology groups the reduced homology groups and denote them by $\bar{H}_{*}$ (resp. for the reduced cohomologies $\bar{H}^{*}$ ). By taking the cylinder of the augmented chain complexes we obtain a new complex $\overline{\mathbb{M}}_{*}$ (resp. $\overline{\mathbb{M}}^{*}$ ).

We will mostly be interested in the groups $H_{p}(\mathbb{M}(S))$ and $H^{p}(\widehat{\mathbb{M}}(S))$ for $p=0,1$. Here is the basic tool for computing them:

Lemma 2.2.7. For the dual sheaves let $S=\operatorname{Spec} k, k=\bar{k}$ in this statement:

1. $\breve{H}_{p}(\underline{\mathbb{X}}) \simeq H_{p}\left(\mathbb{M}_{*}\right)$ for $p \geq 2$ and there is the following exact sequence:

$$
0 \rightarrow H_{1}(\underline{\mathbb{X}}) \rightarrow H_{1}\left(\mathbb{M}_{*}\right) \rightarrow H_{0}(\underline{\text { Fun }}) \rightarrow H_{0}(\underline{\mathbb{X}}) \rightarrow H_{0}\left(\mathbb{M}_{*}\right) \rightarrow 0 .
$$

2. Dually, $H^{p}\left(\widehat{\mathbb{M}}^{*}\right) \simeq \breve{H}^{p}(\underline{\widehat{\mathbb{X}}})$ for $p \geq 2$ and there is the following exact sequence:

$$
1 \rightarrow H^{0}\left(\widehat{\mathbb{M}}^{*}\right) \rightarrow H^{0}(\underline{\widehat{\mathbb{X}}}) \rightarrow H^{0}(\underline{\widehat{\mathrm{Fun}}}) \rightarrow H^{1}\left(\widehat{\mathbb{M}}^{*}\right) \rightarrow H^{1}(\underline{\widehat{\mathbb{X}}}) \rightarrow 1 .
$$

3. $H_{0}\left(\mathbb{M}_{*}\right) \simeq H_{0}(\underline{\mathbb{K}})$ and there is the following exact sequence:

$$
H_{2}(\underline{\mathbb{K}}) \rightarrow H_{0}(\underline{\mathbb{L}}) \rightarrow H_{1}\left(\mathbb{M}_{*}\right) \rightarrow H_{1}(\underline{\mathbb{K}}) \rightarrow 0 .
$$

4. Dually, $H^{0}\left(\widehat{\mathbb{M}}^{*}\right) \simeq H^{0}(\underline{\widehat{\mathbb{K}}})$ and there is the following exact sequence:

$$
1 \rightarrow H^{1}(\underline{\widehat{\mathbb{K}}}) \rightarrow H^{1}\left(\widehat{\mathbb{M}}^{*}\right) \rightarrow H^{0}(\underline{\widehat{\mathbb{L}}}) \rightarrow H^{2}(\underline{\widehat{\mathbb{K}}}) .
$$

Proof. The short exact sequence of complexes

$$
0 \rightarrow C_{*}(\underline{\mathbb{X}}) \rightarrow \mathbb{M}_{*} \rightarrow C_{*}(\underline{\text { Fun }})[-1] \rightarrow 0
$$

of Cech chains gives rise to a long exact sequence on homologies. Part 1) follows from this sequence immediately by application the vanishing Lemma 2.2.4 and the fact that $H_{p}=\breve{H}_{p}$ for $p=0,1$. 
Similarly, we have the following exact sequences:

$$
\begin{aligned}
& 0 \rightarrow \operatorname{Cone}\left(C_{*}(\underline{\text { Fun }}) \rightarrow \operatorname{im} \phi\right) \rightarrow \mathbb{M}_{*} \rightarrow C_{*}(\underline{\mathbb{K}}) \rightarrow 0, \\
& 0 \rightarrow C_{*}(\underline{\mathbb{L}}) \rightarrow C_{*}(\underline{\text { Fun }}) \rightarrow \operatorname{im} \phi \rightarrow 0 .
\end{aligned}
$$

The latter sequence implies that the complexes Cone $\left(C_{*}(\underline{\text { Fun }}) \rightarrow \operatorname{im} \phi\right)$ and $C_{*}(\underline{\mathbb{L}})[-1]$ are quasi-isomorphic. Therefore, in the derived category we have a triangle

$$
C_{*}(\underline{\mathbb{L}})[-1] \rightarrow \mathbb{M}_{*} \rightarrow C_{*}(\underline{\mathbb{K}})
$$

Consequently, it also gives a long exact sequence of homologies. This, together with the fact that $H_{2} \rightarrow \breve{H}_{2}$ is surjective, gives 3). Parts 2) and 4) follow by a dual argument.

Remark 2.2.8. There are also similar exact sequences for the reduced (co)homologies which we skip for brevity.

Definition 2.2.9. Instead of the sheaf of groups $\underline{\text { Fun }}$ in the definition of the complex $\widehat{\mathbb{M}}^{*}$ we can try to use the sheaf of semigroups for multiplication $\widehat{\underline{\text { Fun }}}_{\geq 0}$. The first two terms of the complex $\widehat{\mathbb{M}}_{\geq 0}$ are

$$
\widehat{\mathbb{M}}_{\geq 0}^{0}=C^{0}(\underline{\widehat{\mathbb{X}}})=\widehat{\mathbb{M}}^{0} \quad \text { and } \quad \widehat{\mathbb{M}}_{\geq 0}^{1}=C^{1}(\underline{\widehat{\mathbb{X}}}) \oplus C^{0}\left(\widehat{\operatorname{Fun}}_{\geq 0}\right) .
$$

The group $\widehat{\mathbb{M}}_{\geq 0}^{0}$ naturally acts on $\widehat{\mathbb{M}}_{\geq 0}^{1}$ according to the formula

$$
\hat{x}^{0} \cdot\left(\hat{x}^{1}, \hat{f}^{0}\right)=\left(d \hat{x}^{0} \cdot \hat{x}^{1}, \hat{x}^{0} \hat{f}^{0}\right) .
$$

We will denote by $Z^{1}\left(\widehat{\mathbb{M}}_{\geq 0}^{*}\right)$ the subset of $\widehat{\mathbb{M}}_{\geq 0}^{1}$ of pairs $\left(\hat{x}^{1}, \hat{f}^{0}\right)$ such that

$$
d \hat{x}^{1}=0 \quad \text { and } \quad \hat{f}_{i_{1}}^{0}=\hat{x}_{i_{1} i_{2}}^{1} \hat{f}_{i_{2}}^{0} \text { in } \operatorname{Fun}\left(X_{i_{1}}, \mathcal{O}_{S}(S)\right) \text { for all } i_{1}>i_{2} .
$$

Example 2.2.10. Let $\Delta$ be the complex of lattice polytopes as on the picture. Explicitly, the coordinates of the vertices are $(0,0),(0,4),(4,0)$, $(1,1),(1,2)$ and $(2,1)$. From the long exact sequence of homologies associated to

$$
0 \rightarrow \underline{X} \rightarrow \underline{\mathbb{X}} \rightarrow \mathbb{Z} \rightarrow 0
$$

taking into account that $H_{p}(\Delta, \mathbb{Z})=0$ for $p>0$ and $H_{0}(\Delta, \mathbb{Z})=\mathbb{Z}$ (since $|\Delta|$ is contractible), we obtain $H_{p}(\Delta, \underline{\mathbb{X}})=H_{p}(\Delta, \underline{X})$ for $p>0$ and $H_{0}(\Delta, \underline{\mathbb{X}})=$ 
$H_{0}(\Delta, \underline{X}) \oplus \mathbb{Z}$. An explicit computation of Cech homologies shows that $H_{0}(\Delta, \underline{X}) \simeq \mathbb{Z}^{3}, H_{1}(\Delta, \underline{X}) \simeq \mathbb{Z}$ and higher homologies vanish.

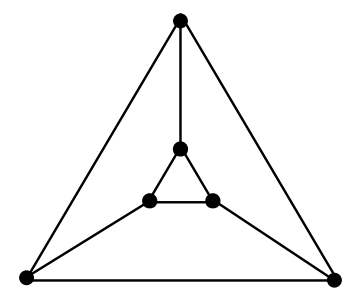

Example 2.2.11. Consider a complex $\Delta$ consisting of four squares meeting at the origin, with the outside vertices $( \pm 1, \pm 1),(0, \pm 2),( \pm 2,0)$. The group $C_{1}(\underline{X})$ is the direct sum of four copies of $\mathbb{Z}$ corresponding to four common sides of the squares. The image $B_{0}$ of this group in $C_{0}$ contains the element $\{(2,0),(-2,0),(0,2),(0,-2)\}$ but not the half of it. Therefore, $H_{0}(\underline{X})=C_{0} / B_{0}$ has a 2 -torsion. If $\Delta^{(p)}, p>0$, denotes the direct product of $\Delta$ with $p$ copies of the complex from the example 2.2.10 then by the Küneth formula we see that $H_{p}\left(\Delta^{(p)}, \underline{X}\right)=H_{p}\left(\Delta^{(p)}, \underline{\mathbb{X}}\right)$ has 2-torsions.

2.3. Affine stable toric varieties. The first example is an ordinary affine toric variety over an algebraically closed field $k$. For a rational polyhedral cone $\omega \subset X_{\mathbb{R}}$, which need not be maximal-dimensional, let $S_{\omega}=\omega \cap X$ be the saturated semigroup of lattice elements in $\omega$, and denote the semigroup algebra $k\left[S_{\omega}\right]$ by $R_{\omega}$. Then $P_{\omega}=\operatorname{Spec} R_{\omega}$ is an affine torus embedding, and it is well-known that it is normal (hence seminormal) and that our condition on the stabilizers is satisfied for the standard torus action $T \curvearrowright P_{\omega}$. We are now going to generalize this.

2.3.1. Let $\Omega$ be a cone complex referenced by $X$. For each cone $\omega_{i}$ we have an associated semigroup and a semigroup algebra

$$
S_{i}=X \cap \omega_{i}=X_{i} \cap \omega_{i}, \quad R_{i}=k\left[S_{i}\right]=\oplus_{s \in S_{i}} k \zeta^{s} .
$$

To each semigroup $S_{i}$ we will add one more element $\infty$ with the property $s+\infty=\infty$ for all $s \in S_{i}$. We will make a convenient but nonstandard choice: in the corresponding semigroup algebra we will set formally $\zeta^{\infty}=0$. To avoid confusion with the ordinary semigroup algebra $k\left[S_{i} \cup \infty\right]$ we will call this algebra $k_{\infty}\left[S_{i} \cup \infty\right]$. Note that the collection $\left\{S_{i}\right\}$ is naturally a cosheaf on $\Omega_{\text {ord }}$ : for two cones $\omega_{i_{1}}$ and $\omega_{i_{2}}$ with $i_{1}>i_{2}$ (i.e. $\omega_{i_{1}}$ is a face of $\omega_{i_{2}}$ ) one has an embedding $S_{i_{1}} \hookrightarrow S_{i_{2}}$. Since $S_{i_{2}} \backslash S_{i_{1}}$ is a semigroup ideal, we can make the collection $\left\{S_{i} \cup \infty\right\}$ into a sheaf on $\Omega_{\text {ord }}$ : for $i_{1}>i_{2}$ one has the Reese homomorphism $S_{i_{2}} \cup \infty \rightarrow S_{i_{1}} \cup \infty$ defined by

$$
S_{i_{2}} \ni s \mapsto \begin{cases}s & \text { if } s \in S_{i_{1}} \\ \infty & \text { otherwise. }\end{cases}
$$


The Reese semigroup homomorphism induces an epimorphism of semigroup algebras $\operatorname{pr}_{i_{2} i_{1}}: R_{i_{2}} \rightarrow R_{i_{1}}$. We will denote these induced (co)sheaves of semigroups and algebras by $\underline{\mathrm{S}}[\Omega]$ and $\underline{\mathrm{R}}[\Omega]$ respectively.

Lemma 2.3.2. 1 . In the category of sets $\underset{\lim }{\longleftarrow}\left(S_{i} \cup \infty\right)=\left(\underline{\lim } S_{i}\right) \cup \infty$.

2. For any ring $k($ including $k=\mathbb{Z})$ in the category of $k$-algebras,

$$
H^{0}(\Omega, \underline{\mathrm{R}}[\Omega])=k_{\infty}\left[H^{0}(\Omega, \underline{\mathrm{S}}[\Omega])\right] .
$$

This is a free $k$-module with the basis $\left\{\zeta^{s}, s \in \lim S_{i}\right\}$ and the multiplication

$$
\zeta^{s} \cdot \zeta^{t}= \begin{cases}\sum_{\zeta^{s+t}} & \text { for all } S_{i} \text { so that } s, t \in S_{i} \\ 0 & \text { otherwise. }\end{cases}
$$

Proof. All parts are immediate from the definitions.

Definition 2.3.3. Define an algebra $R[\Omega]=H^{0}(\Omega, \underline{\mathrm{R}}[\Omega])$ and the affine variety $P[\Omega]$ as $\operatorname{Spec} R[\Omega]$.

There is a natural torus action on $R[\Omega]$ and $P[\Omega]$ : for each $t \in T=$ $\operatorname{Hom}\left(X, \mathbb{G}_{m}(k)\right)$ and $u \in S_{\omega}$ one has $t . \zeta^{u}=t(\rho u) \zeta^{u}$.

Example 2.3.4. Divide the line $\mathbb{R} \supset \mathbb{Z}$ into three cones: two half-lines $\omega_{1}$ and $\omega_{2}$ and the origin $\omega_{12}$. One has $R_{1}=k[x], R_{2}=k[y], R_{\omega_{12}}=k$. Then $H^{0}(\Omega, \underline{\mathrm{R}}[\Omega])=k[x, y] /(x y)$. The variety $P[\Omega]$ is the union of two transversally intersecting lines.

Remark 2.3.5. In the case when the function $\rho:|\Omega| \rightarrow X_{\mathbb{R}}$ is injective, the algebra $R[\Omega]$ was introduced by R. Stanley in [Sta2, $\S 4]$.

Next, we define a twisted version of the above. Consider a collection $\left\{t_{i_{1} i_{0}} \in T_{i_{0}}\right\}$ where $\left(i_{1}, i_{0}\right)$ goes over all pairs of indices with $i_{0}>i_{1}$ so that for all $i_{0}>i_{1}>i_{2}$ one has

$$
t_{i_{2} i_{0}}=\varphi_{i_{1} i_{0}}\left(t_{i_{2} i_{1}}\right) \cdot t_{i_{1} i_{0}} \text { in } T_{i_{0}}
$$

(this can be supplemented by the elements $t_{i i}=1$ ). A collection like this is equivalent to a cocycle $t \in Z^{1}\left(\mathcal{U}_{\text {std }}, \underline{T}\right)$. This cocycle defines a sheaf $\underline{\mathrm{R}}[\Omega, t]$ with the same stalks $R_{i}$ and twisted homomorphisms

$$
p_{i_{1} i_{0}}=t_{i_{1} i_{0}} \cdot \operatorname{pr}_{i_{1} i_{0}} .
$$

Definition 2.3.6. Similarly, we define an algebra and a variety

$$
R[\Omega, t]=H^{0}(\Omega, \underline{\mathrm{R}}[\Omega, t]), \quad P[\Omega, t]=\operatorname{Spec} R[\Omega, t] .
$$

As before, it comes with a natural $T$-action. 
The algebras $R[\Omega, t]$ are a particular case of "glued algebras" studied by Dayton and Weibel in [DW] and, on the other hand, of sections of rings of flasque (flabby) sheaves of integral algebras studied by Yuzvinsky in [Yuz]. Thus, we can describe the precise structure of $R$ 's and $P$ 's by specializing and combining the quite general results of these papers. We note that Dayton and Weibel work with "almost posets" which are slightly more general than what we need, and Yuzvinsky works with the semilattices which are slightly less general than what we need. In most cases, the general case can be deduced from the special case of a semilattice by a simple trick.

Remark 2.3.7. If the reference function $\rho:|\Omega| \rightarrow X_{\mathbb{R}}$ is one-sheeted, i.e. injective, then $\Omega$ is a semilattice.

LEmma 2.3.8. The sheaf $\underline{\mathrm{R}}[\Omega, t]$ is flabby (flasque).

Proof. If $\Omega$ is a semilattice, this is an application of the criterion in [Yuz, Cor.1.11] - one has to check two properties for a semigroup algebra $k\left[S_{\omega}\right]$ corresponding to a cone $\omega$ and a collection of ideals corresponding to its faces, which is immediate.

The general case is reduced to the case of a semilattice by subdividing some cones into several "dummy" cones and assigning to these interior cones the same semigroups and identical connecting homomorphisms.

Corollary 2.3.9. As a topological space,

$$
P[\Omega, t]=\underline{\lim } \operatorname{Spec} k\left[S_{i}\right]=\lim _{\longrightarrow} \operatorname{Spec} P_{i}
$$

In particular, the irreducible components of $P[\Omega, t]$ are the toric varieties $P_{i_{0}}$ for maximal cones $\omega_{i_{0}}$, they intersect along unions of smaller toric varieties $P_{i}$ etc.

Proof. Since the sheaf $\underline{\mathrm{R}}[\Omega, t]$ is flabby, for each $i$ there is a prime ideal $P_{i}$ such that $R_{i}=R[\Omega, t] / P_{i}$. With this in mind, [DW, Thm.1.2] and the previous lemma imply that the ring $R[\Omega, t]$ satisfies the condition (CRT) 1 of [DW, p. 37] for all $l \geq 2$. The statement then is proved in [DW, Prop. 1.6].

Corollary 2.3.10. $R[\Omega, t]$ is seminormal.

Proof. By [DW, Thm.1.8].

Lemma 2.3.11. $P[\Omega, t]=\operatorname{Spec} R[\Omega, t]$ is an affine stable toric variety (STV). It is weakly normal as well.

Proof. $R[\Omega, t]$ is obviously reduced. The only property that we have not yet checked is that the stabilizer of every point is connected and reduced. But every point lies in one of the irreducible components, and for normal toric varieties this property is well-known. For the last statement, consider 
a bijection $f: P^{\prime} \rightarrow P$ inducing an isomorphism on the residue fields for all generic points. Since every irreducible component of $P$ is normal, $f$ has to be an isomorphism on each of them, and so it has to induce an isomorphism on the residue fields of each scheme point $x \in P$. Hence, the seminormality implies the weak normality in this case.

2.3.12. Now let $P$ be any affine STV. First of all, note that for each $p \in P$, the orbit $\operatorname{orb} p=T p$ is isomorphic to the quotient torus $T_{p}=T / \mathrm{Stab}_{p}$. If $X_{p} \subset X$ is its group of characters then the stabilizer $\mathrm{Stab}_{p}$ is the multiplicative group dual to $X / X_{p}$. Hence, it is connected and reduced $\Leftrightarrow X / X_{p}$ is torsion free; i.e. the sublattice $X_{p} \subset X$ is saturated.

Lemma 2.3.13. Let $P$ be an irreducible affine variety with T-action and only finitely many T-orbits. Assume that for each $p \in P$ the stabilizer Stab $_{p}$ is connected and reduced. Then:

1. The normalization $P^{\nu}$ is isomorphic to an affine torus embedding containing a quotient torus $T^{\prime}$.

2. $\nu: P^{\nu} \rightarrow P$ is bijective and induces isomorphisms on the residue fields of all scheme points.

3. If $P$ is seminormal then $P=P^{\nu}$.

Proof. Say $P=\operatorname{Spec} R$ for a finitely generated $k$-algebra $R$. Let $p$ be an arbitrary point in the dense orbit that is isomorphic to a torus $T^{\prime}$ with a group of characters $X^{\prime}$. The $T$-action on $R$ defines an $X$-grading, and the embedding $T^{\prime} \subset P$ defines an embedding $R \subset k\left[X^{\prime}\right]$ of $X$-graded algebras since $T^{\prime}$ is dense. Therefore, $R$ is isomorphic to a semigroup algebra $k[S]$, where $S$ is a finitely generated sub-semigroup of $X^{\prime}$. The normalization $P^{\nu}$ obviously is Spec $k\left[S^{\text {sat }}\right]$, a torus embedding corresponding to a cone $\omega \subset X_{\mathbb{R}}^{\prime}$. The $T$ orbits of $P$ are in a 1-to-1 correspondence with faces of this cone. Since the stabilizers are connected and reduced, for each face $F$ the semigroup $S \cap F$ generates $F \cap X^{\prime}$ as a group. Hence, $P^{\nu} \rightarrow P$ is bijective and induces an isomorphism $k(x) \simeq k\left(x^{\prime}\right)$ for every scheme point $x^{\prime} \in X^{\nu}$ with $\nu\left(x^{\prime}\right)=x$. It has to be an isomorphism if $P$ is seminormal.

Therefore, an irreducible affine STV is simply an affine toric variety corresponding to a cone $\omega \subset X_{\mathbb{R}}$.

ThEOREM 2.3.14. Every affine STV $P$ is isomorphic to one of $P[\Omega, t]$.

Proof. $P$ is a union of finitely many $T$-orbits $\mathrm{Orb}_{i}$. Let $P_{i}$ be the closure of $\mathrm{Orb}_{i}$. It is irreducible, our condition on the stabilizers is satisfied, and so by the above it defines a cone $\omega_{i}$ in $X$. A natural order by inclusion on $P_{i}$ 's makes 
the index set into a poset. Further, since $P_{i}^{\nu} \rightarrow P_{i}$ is bijective and $T$-orbits on an affine toric variety correspond to faces of the cone, if $P_{i_{1}} \subset P_{i_{2}}$ then the cone $\omega_{1}$ is a face of $\omega_{2}$. An intersection of two $P_{i}$ 's is a union of (closures) of other orbits, i.e. $P_{j}$ 's. Hence, the variety $P$ defines uniquely a complex of cones $\Omega$.

Further, for each $i$ choose a point $p_{i} \in \mathrm{Orb}_{i}$, i.e. an origin in the subvariety $P_{i}$. This choice identifies the normalization $P_{i}^{\nu}$ with a standard affine toric variety $\operatorname{Spec} S_{i}$. It also gives an origin in each lower dimensional $T$-orbit on Spec $S_{i}$ : it is $\lim _{1-P S} p_{i}$ for any 1-parameter subgroup for which the limit lands in that orbit. Since $P_{i}^{\nu} \rightarrow P_{i}$ is an isomorphism when restricted to any $T$-orbit, for all $i_{1}>i_{2}$ in $\operatorname{Orb}_{i_{1}}$ we have two "origins" which differ by the torus action: for a uniquely defined $t_{i_{1} i_{2}} \in T_{i_{1}}$ one has

$$
\lim _{1-\mathrm{PS}} p_{i_{2}}=t_{i_{1} i_{2}}^{-1} \cdot p_{i_{1}}
$$

The collection $\left\{t_{i_{1} i_{2}}\right\}$ is obviously compatible with respect to triples $i_{1}>i_{2}>i_{3}$ and so is equivalent to a cocycle $t \in Z^{1}\left(\mathcal{U}_{\text {std }}, \underline{T}\right)$.

Say $P=\operatorname{Spec} R$. The collection of morphisms $P_{i}^{\nu} \rightarrow P$ is equivalent to a collection of homomorphisms $R \rightarrow R_{i}$ and hence to a homomorphism from $R$ to $\lim R_{i}=R[\Omega, t]$. By the construction and Lemma 2.3.9 the dual morphism $P[\Omega, t] \rightarrow P$ is a bijection and by 2.3.13.2 it induces an isomorphism on residue fields. Since $P$ is seminormal, it has to be an isomorphism.

2.3.15. This lemma establishes a 1-to-1 correspondence between the "marked" affine STVs and the data $\left[\Omega, t^{1}\right]$. The standard origin $p_{i}$ on Spec $k\left[S_{i}\right]$ is the same as a homomorphism $p_{i}: k\left[S_{i}\right] \rightarrow k$ sending each $\zeta^{s}$ to 1 . Via the connecting homomorphism $p_{i_{2} i_{1}}=t_{i_{2} i_{1}} \cdot \mathrm{pr}_{i_{2} i_{1}}$ we see that $\lim _{1-\mathrm{PS}} p_{i_{2}}: t_{i_{2} i_{1}} \cdot \zeta^{S} \mapsto 1$; hence $\lim _{1-\mathrm{PS}} p_{i_{2}}=t_{i_{1} i_{2}}^{-1} \cdot p_{i_{1}}$.

Definition 2.3.16. Consider the "framed" category $M^{\mathrm{fr}}[\Omega](k)$ of all affine STVs over a fixed algebraically closed field $k$ corresponding to a given complex $\Omega$ with the action of a fixed torus $T$, in which we let the arrows be the isomorphisms giving the identity on $T$. As a version, we can also consider the category $M[\Omega](k)$ where we allow arbitrary automorphisms $T \stackrel{\sim}{\rightarrow} T$ (these are in a 1-to-1 correspondence with automorphisms of $X$, i.e. elements of $\operatorname{GL}(X)$.

We will postulate them to be small categories. Such a category is called a groupoid and it is given by two sets: $U$ - the set of objects and $R$ - the set of arrows and a map $j=(b, e): R \rightarrow U \times U$ associating to an arrow its beginning and end, and another map $e: U \rightarrow R$ associating to an object the identity arrow. These maps should satisfy the well-known axioms. This groupoid will be denoted $[U / R]$. In particular, if $G$ is an abstract group acting 
on a set $U$ then $[U / G]$ will denote the groupoid obtained by taking $R=G \times U$ and $R \rightarrow U \times U$ defined as $(g, u) \mapsto(u, g . u)$. The symbol 1 will denote a 1-point set.

Lemma 2.3.17. If $\Omega$ consists of a single cone $\omega$ and its faces then the groupoid $M^{\mathrm{fr}}[\Omega](k)$ is equivalent to $\left[1 / T_{\omega}(k)\right]$ and $M[\Omega](k)$ is equivalent to $\left[1 / T_{\omega}(k) \ltimes \operatorname{Sym} \omega\right]$, where the latter means the extension of the group $T_{\omega}(k)$ by the subgroup of $\mathrm{GL}(X)$ of symmetries of the cone $\omega$.

Proof. Trivial.

Theorem 2.3.18. In general, $M^{\mathrm{fr}}[\Omega](k)$ is equivalent to

$$
\left[Z^{1}\left(\mathcal{U}_{\mathrm{std}}, \underline{T}\right) / C^{0}\left(\mathcal{U}_{\mathrm{std}}, \underline{T}\right)\right] \sim\left[H^{1}(\Omega, \underline{T}) / H^{0}(\Omega, \underline{T})\right]
$$

with the last group acting trivially. In other words, the set of isomorphism classes of framed affine STVs corresponding to a complex $\Omega$ is $H^{1}(\Omega, \underline{T})$ and for each $t, \operatorname{Aut} P[\Omega, t]=H^{0}(\Omega, \underline{T})$. The groupoid $M[\Omega](k)$ is equivalent to

$$
\left[Z^{1}\left(\mathcal{U}_{\text {std }}, \underline{T}\right) / C^{0}\left(\mathcal{U}_{\text {std }}, \underline{T}\right) \ltimes \operatorname{Sym} \Omega\right] \sim\left[H^{1}(\Omega, \underline{T}) / H^{0}(\Omega, \underline{T}) \ltimes \operatorname{Sym} \Omega\right] .
$$

Proof. By 2.3.15, the marked STVs over $k$ are classified by the set $Z^{1}\left(\mathcal{U}_{\text {std }}, \underline{T}\right)$ and the automorphism groups of marked STVs are trivial. The groupoid of unmarked STVs is obtained by dividing this set by the equivalence relation induced by choosing different origins. That is obviously given by the action of $C^{0}\left(\mathcal{U}_{\mathrm{std}}, \underline{T}\right)$, and the statement follows.

As mentioned above, the rings $R[\Omega, t]$ generalize the rings $R[\Omega]$ introduced by Stanley for a complex of cones lying inside $X_{\mathbb{R}}$, and on the other handthe Stanley-Reisner rings of simplicial complexes. As in these particular cases, the singularities of $P[\Omega, t]=$ Spec $R[\Omega, t]$ strongly depend on the topology of the geometric realization $|\Omega|$. See Definition 1.1.30 for the notions of CM and locally CM complexes.

TheOREM 2.3.19. Assume that the complex $\Omega$ is a semilattice. If $\Omega$ is CM then $P[\Omega, t]$ is CM.

Proof. This is an application of a theorem of Yuzvinsky [Yuz, 5.1] and the proof proceeds in the same way as that of $[\mathrm{Sta} 2,4.6]$ which is a special case.

For each $q>0$ and each $q$-dimensional cone $\omega$, choose $s_{\omega} \in \omega^{0}$. This defines an element $\zeta^{s_{\omega}}$ in $k\left[S_{\omega}\right]$ and so also in $R[\Omega, t]$. Let $r_{q}=\sum_{\operatorname{dim} \omega=d+1-q} \zeta^{s_{\omega}}$, where $d=\operatorname{dim} \Omega$. Then $r_{1}, \ldots, r_{d}$ is a standard system of parameters as defined in [Yuz], and the statement follows from Theorem 5.1. there. 
Remark 2.3.20. Assume that $\Omega$ is not a semilattice but is a quotient of another complex $\Omega^{\prime}$ that is a semilattice and CM, by a finite group $G$ whose order is coprime to chark. Then $P[\Omega, t]=P\left[\Omega^{\prime}, t^{\prime}\right] / G$ and so is CM as well. I do not know whether this can be done for any CM complex of cones.

On the other hand, it seems that the results of Yuzvinsky can be extended to the general (nonsemilattice) case.

\subsection{Polarized stable toric varieties with a linearized line bundle.}

2.4.1. Affine torus embeddings over a closed field correspond in a 1-to-1 way to finitely generated rational polyhedral cones in $X_{\mathbb{R}}$. Analogously, projective torus embeddings $(P, L)$ together with an extended action $T \curvearrowright L$ correspond 1-to-1 to lattice polytopes in $X_{\mathbb{R}}$. Let us recall this connection.

Let $\delta \subset X_{\mathbb{R}}$ be a polytope with vertices in $X$. Let Cone $\delta \subset \mathbb{X}_{\mathbb{R}}$ be the cone over $\delta$ lying in the hyperplane $\left(1, X_{\mathbb{R}}\right)$, and denote the semigroup $\mathbb{X} \cap$ Cone $\delta$ by $S_{\delta}$. Then $P_{\delta}=\operatorname{Proj} k\left[S_{\delta}\right]$ is a projective torus embedding and $L_{\delta}=\mathcal{O}(1)$ is a linearized ample invertible sheaf. One has a canonical identification

$$
\oplus_{d \geq 0} H^{0}\left(P_{\delta}, L_{\delta}^{d}\right)=k\left[S_{\delta}\right]=R_{\text {Cone } \delta} .
$$

The affine cone Spec $R_{\text {Cone } \delta}$ is an affine toric variety for the action of the torus $\mathbb{T}=\mathbb{G}_{m} \oplus T$. The complement of the apex of this cone is the $\mathbb{G}_{m}$-torsor $\mathbf{L}_{\delta}^{-1}$ which is obtained from the line bundle $L_{\delta}^{-1}$, considered as a variety, by removing the zero section.

Definition 2.4.2. Given a complex of lattice polytopes $\Delta$ referenced by $X$ and a cohomology class $[\tau] \in H^{1}(\Delta, \mathbb{T})$ we define a projective variety $P[\Delta, \tau]$ as the Proj of $R[\Delta, \tau]=R[$ Cone $\Delta, \tau]$ and an ample sheaf $L[\Delta, \tau]$ on it as $\mathcal{O}(1)$. The corresponding $\mathbb{G}_{m}$-torsor will be denoted $\mathbf{L}[\Delta, \tau]$.

Remark 2.4.3. The algebras $k\left[S_{\delta}\right]$ and $R[\Delta, \tau]$ are not necessarily generated in degree 1. However, it is easy to see that each of them is finite over the subalgebra generated by elements of degree 1 . In this situation $\mathcal{O}(1)$ is an invertible sheaf; see, for example, [Mor].

THEOREM 2.4.4. The pair $(P, L)[\Delta, \tau]$ is a polarized stable toric variety with a T-linearized action on $L$. It is weakly normal as well.

Proof. Take a polytope $\delta \subset\left(1, X_{\mathbb{R}}\right)$ and an element $u \in S_{\delta}$. It is easy to see that the localization of the algebra $R_{\delta}$ at $\zeta^{u}$ is the semigroup algebra $k\left[\mathbb{X} \cap \operatorname{Star}_{u}\right.$ Cone $\left.\delta\right]$ and the subalgebra in the latter of elements of degree $0-$ the semigroup algebra $k\left[X \cap \operatorname{Star}_{u}\right.$ Cone $\left.\delta\right]=k\left[S_{\operatorname{Star}_{u} \delta}\right]$.

Given two polytopes $\delta_{i_{1}}>\delta_{i_{2}}$, a homomorphism $\tau_{i_{2} i_{1}} \cdot \operatorname{pr}_{i_{2} i_{1}}: k\left[S_{\text {Cone } \delta_{i_{2}}}\right] \rightarrow$ $k\left[S_{\text {Cone } \delta_{i_{2}}}\right]$ induces the homomorphism $t_{i_{2} i_{1}} \cdot \operatorname{pr}_{i_{2} i_{1}}: k\left[S_{\mathrm{Star}_{u} \delta_{i_{2}}}\right] \rightarrow k\left[S_{\mathrm{Star}_{u} \delta_{i_{2}}}\right]$, where $t_{i_{2} i_{1}}$ is the image of $\tau_{i_{2} i_{1}}$ under the natural projection $\mathbb{T}_{i_{1}} \rightarrow T_{i_{1}}$. Since the inverse limit commutes with the localization and the subalgebra is of 
degree 0 , we see that

$$
\left(R[\Delta, \tau]\left[\left(\zeta^{u}\right)^{-1}\right]\right)_{0}=R\left[\operatorname{Star}_{u} \Delta, t\right]
$$

The algebra $R[\Delta, \tau]$ is finite over the subalgebra generated by $\zeta^{u}$ for all elements $u$ of degree 1 which appear as vertices of some polytopes $\delta_{i}$. Therefore, $\operatorname{Proj} R[\Delta, \tau]$ is covered by finitely many affine STVs, and so is a projective STV. The $\mathbb{X}$-grading on $R[\Delta, \tau]$ defines the $\mathbb{T}$-action on $L=\mathcal{O}(1)$. This proves the statement.

Corollary 2.4.5. As a topological space,

$$
P[\Delta, \tau]=\underset{\lim }{\longrightarrow} P_{i}
$$

In particular, the irreducible components of $P[\Delta, \tau]$ are the projective toric varieties $P_{i_{0}}$ for maximal polytopes $\delta_{i_{0}}$; they intersect along smaller $P_{i}^{\prime}$ 's etc. The restriction of $L[\Delta, \tau]$ to each $P_{i}$ is isomorphic to $L_{i}$.

Proof. This is immediate from 2.3.9.

2.4.6. Let $P$ be an arbitrary projective variety with the $T$-action and $L$ be an ample line bundle with the extended $T$-action. On the other hand, we have a natural scalar $\mathbb{G}_{m}(k)$-action on $L$. Because $H^{0}(P, L)=k$, the only automorphisms of $L$ covering the identity on $P$ are in $\mathbb{G}_{m}(k)$. Therefore, the two actions generate the action by a bigger group $\mathbb{T}$ which is the extension of $\mathbb{G}_{m}(k)$ by $T$. Every such extension is commutative, so $\mathbb{T}=\mathbb{G}_{m}(k) \oplus T$.

THEOREM 2.4.7. Every polarized stable toric variety $(P, L)$ with a linearized ample sheaf $L$ is isomorphic to one of polarized varieties $P[\Delta, \tau]$ for a complex $\Delta$ of lattice polytopes referenced by $X$.

Proof. Consider the algebra $R=\oplus_{d \geq 0} H^{0}\left(P, L^{d}\right)$. Then $P=\operatorname{Proj} R$, and Spec $R$ is an affine cone over $P$. Note that the maximal ideal $R_{+}$of elements of positive degree is not an embedded prime. Since $P$ is reduced, $R$ is reduced. The algebra $R$ has a natural $\mathbb{T}$-action. We claim that the seminormalization of $R$ is isomorphic to one of the algebras $R[\Delta, t]$. Indeed, $\operatorname{Spec} R^{\mathrm{sn}} \rightarrow \operatorname{Spec} R$ is an isomorphism except possibly at the apex since the complement of the apex is the $\mathbb{G}_{m}$-torsor $\mathbf{L}^{-1}$ over a seminormal variety. Hence, our condition on the orbits is satisfied and $\operatorname{Spec} R^{\mathrm{sn}}$ is an affine STV. Since the two affine varieties differ possibly only at the apex, it follows that $P=\operatorname{Proj} R=\operatorname{Proj} R[\Delta, t]$ and $L=\mathcal{O}(1)$. 
Remark 2.4.8. The results of the next subsection will imply that indeed $R \simeq R[\Delta, \tau]$.

2.4.9. As in the affine case, there is a unique isomorphism $P \stackrel{\sim}{\rightarrow} P[\Delta, \tau]$ if we mark the origins $p_{i}$ in each $\mathbb{T}$-orbit on $P$, i.e. in each $\mathbb{G}_{m}$-torsor $\mathbf{L}_{i}^{-1}$ over $P_{i}$ (the 0-dimensional orbit, the apex, can be omitted).

Definition 2.4.10. For a complex $\Delta$ of lattice polytopes referenced by a lattice $X, M[\Delta](k)$ will denote the groupoid of polarized STVs with a linearized line bundle $L$ over $k=\bar{k}$ in which the arrows are the isomorphisms identical on $T$. As before, $M^{\mathrm{fr}}[\Delta](k)$ is a similar category in which we allow the isomorphisms which are not necessarily identities on $T$.

We skip the proof of the following theorem since it is almost exactly the same as in the affine case.

TheOREM 2.4.11. The groupoid $M^{\mathrm{fr}}[\Delta](k)$ is equivalent to

$$
\left[Z^{1}\left(\mathcal{U}_{\text {std }}, \underline{\mathbb{T}}\right) / C^{0}\left(\mathcal{U}_{\text {std }}, \underline{\mathbb{T}}\right)\right] \sim\left[H^{1}(\Delta, \underline{\mathbb{T}}) / H^{0}(\Delta, \underline{\mathbb{T}})\right]
$$

with the last group acting trivially. In other words, the set of isomorphism classes of framed polarized STVs with the linearized action corresponding to a complex $\Delta$ is $H^{1}(\Delta, \mathbb{T})$ and for each $\tau$ one has $\operatorname{Aut} P[\Delta, \tau]=H^{0}(\Delta, \mathbb{T})$. The groupoid $M[\Delta](k)$ is equivalent to

$$
\left[Z^{1}\left(\mathcal{U}_{\text {std }}, \underline{\mathbb{T}}\right) / C^{0}\left(\mathcal{U}_{\text {std }}, \underline{\mathbb{T}}\right) \ltimes \operatorname{Sym} \Delta\right] \sim\left[H^{1}(\Delta, \mathbb{T}) / H^{0}(\Delta, \underline{\mathbb{T}}) \ltimes \operatorname{Sym} \Delta\right] .
$$

Concerning the singularities, we have a statement similar to the affine case.

LEMma 2.4.12. Assume that the complex $\Delta$ is a semilattice. If $\Delta$ is locally $\mathrm{CM}$ then $P[\Omega, t]$ is $\mathrm{CM}$.

Proof. Indeed, each cone complex $\operatorname{Star}_{v} \Delta, v \neq 0$, in this case is CM.

2.5. Cohomologies of polarized STVs.

Theorem 2.5.1. If $(P, L)=(P, L)[\Delta, \tau]$ then

1. $H^{p}(P, L)=0$ for $p>0$;

2. $H^{0}(P, L)=\pi^{-1}(\tau)$, where $\pi: Z^{1}\left(\widehat{\mathbb{M}}_{\geq 0}\left[\Delta, \rho^{-1} X\right]\right) \rightarrow Z^{1}(\underline{\widehat{\mathbb{X}}})$ is the natural projection, and $h^{0}(P, L)=\# \rho^{-1} X$, the number of integral points in $|\Delta|$.

Corollary 2.5.2. For $(P, L)=(P, L)[\Delta, \tau]$ there is a canonical isomorphism of graded algebras

$$
\oplus_{d \geq 0} H^{0}\left(P, L^{d}\right)=R[\Delta, \tau]
$$


A similar result in a special case can be found in [AN]. The proof will be based on the following two lemmas.

Lemma 2.5.3. For any subcomplex $\Omega^{\prime} \subset \Omega$ (resp. $\Delta^{\prime} \subset \Delta$ ), in other words for any open in the order topology subset there exists a closed affine (resp. polarized) subvariety $P\left[\Omega^{\prime}, t^{\prime}\right] \subset P[\Omega, t]$ (resp. $\left.P\left[\Delta^{\prime}, \tau^{\prime}\right] \subset P[\Delta, \tau]\right)$.

Proof. The projective case follows from the affine since $P[\Delta, \tau]$ is covered by $P[\Omega, t]$ 's. The cocycle $t^{\prime}$ is defined by restricting $t$ : for each cone $\omega_{i^{\prime}} \in \Omega^{\prime}$ choose and fix a cone in $\Omega$ such that $\omega^{\prime} \subset \omega$. If $\omega_{i_{1}^{\prime}} \subset \omega_{i_{1}}$ and $\omega_{i_{2}^{\prime}} \subset \omega_{i_{2}}$ then $t_{i_{1}^{\prime} i_{2}^{\prime}}^{\prime}$ is the image of $t_{i_{1} i_{2}}$. Define the map $f: R[\Omega, t] \rightarrow\left[\Omega^{\prime}, t^{\prime}\right]$ by the formula

$$
\zeta^{u} \mapsto \begin{cases}\zeta^{u} & \text { if } u \in S_{\omega_{\omega^{\prime}}} \subset S_{\omega_{i}} \\ 0 & \text { if } u \notin\left|\Omega^{\prime}\right| .\end{cases}
$$

Clearly, $f$ is well defined, is an algebra homomorphism and is surjective. This gives a closed embedding.

2.5.4. Now choose an open cover $\mathcal{U}=\left\{U_{i_{0}}=U_{\geq i_{0}}\right\}$ of $\Omega_{\text {ord }}$ (resp. of $\left.\Delta_{\text {ord }}\right)$. For each of the open subsets $U_{i_{0}} \cap \cdots \cap U_{i_{q}}$ denote the corresponding closed subscheme by $P_{i_{0} \ldots i_{q}}$. (Warning: if $\mathcal{U} \neq \mathcal{U}_{\min }$, the subschemes $P_{i_{0}}$ are not necessarily irreducible.) We will identify the sheaf $\mathcal{O}_{P_{i_{0} \ldots i_{q}}}$ with its direct image under the embedding $P_{i_{0} \ldots i_{q}} \subset P$. Order the elements $\left\{i_{s}\right\}$ somehow (this has nothing to do with the partial order on $I)$. For each collection $i_{0}<\ldots<i_{q}$ define a homomorphism $d: \mathcal{O}_{P_{i_{0} \ldots \hat{i}_{s} \ldots i_{q}}} \rightarrow \mathcal{O}_{P_{i_{0} \ldots i_{q}}}$ as the restriction $\times(-1)^{s}$. Putting these all together, we obtain a complex

$$
0 \rightarrow \mathcal{O}_{P} \rightarrow \oplus \mathcal{O}_{P_{i_{0}}} \rightarrow \oplus \mathcal{O}_{P_{i_{0} i_{1}}} \rightarrow \cdots
$$

in which clearly $d \circ d=0$.

Lemma 2.5.5. For $P=P[\Omega, t]$ (resp. $P[\Delta, \tau]$ ) and any closed cover $\mathcal{U}$ the above complex is an exact resolution of $\mathcal{O}_{P}$ by the sheaves of $\mathcal{O}_{P}$-modules.

Proof. As before, the projective case follows from the affine. In the affine case, each of the sheaves is represented by an algebra splitting into a direct sum of 0 - or 1 -dimensional vector spaces $V_{x}$, one for each $x \in \rho^{-1} X$. The exactness is sufficient to check on each of the $V_{x}$, which reduces to the acyclicity of the complex when we compute the reduced cohomologies $\bar{H}^{p}\left(D_{n-1}, k\right)$ of an $(n-1)$ dimensional disk using the Leray covering by simplices $D_{n-1}^{(i)}, i=0, \ldots, n$. Hence, we have an exact sequence of commutative groups. These groups are $R$-modules by the previous lemma.

Proof of Theorem 2.5.1. We will prove this statement by induction on the dimension and the number of minimal polytopes in $\Delta$, the case of one polytope, i.e. an ordinary projective toric variety being standard (see for example [Oda], 
[Ful]). In the above resolution take $\mathcal{U}=\mathcal{U}_{\text {min }}$. The cohomologies of $L$ can be computed as the hypercohomologies of the above resolution twisted by $L$. By the induction assumption, all the higher cohomologies of $\mathcal{O}_{P_{i_{0} \ldots i_{q}}} \otimes L$ vanish, so that we are reduced to the complex of $H^{0}$ 's which are also known. Looking at each space $V_{x}$, we see that this complex is exact except for the first term. The kernel of the first homomorphism consists of the collections $\left\{f_{i_{0}} \in H^{0}\left(P_{i_{0}}, L\right)\right\}$ such that for all $i_{0}, i_{1}$ one has in $H^{0}\left(P_{i_{0} i_{1}}, L\right)$ :

$$
f_{i_{0}} t_{i_{0}, i_{0} i_{1}}^{-1}=f_{i_{1}} t_{i_{1}, i_{0} i_{1}}^{-1}, \quad \text { i.e. } \quad f_{i_{0}}=f_{i_{1}} t_{i_{1} i_{0}}^{-1},
$$

which is precisely $\pi^{-1}(\tau)$.

The same argument and the fact that on a projective toric variety higher cohomologies of the structure sheaf vanish give:

Theorem 2.5.6. $H^{p}(P[\Delta, \tau], \mathcal{O})=H^{p}(|\Delta|, k)$.

2.6. Stable toric pairs. A category of pairs $(P, \Theta)$ of varieties and Cartier divisors on them is equivalent to the category $(P, L, \theta)$, where $L=\mathcal{O}(\Theta)$ and $0 \neq \theta \in H^{0}(P, L)$ is an equation of $\Theta$. In this section we deal with the case when $L$ is $T$-linearized. To classify the stable toric pairs in this case we have to add to the data of the previous subsection an equation $\theta$ so that $\Theta$ does not contain any $T$-orbits entirely. We will start with the case when $P$ is irreducible, i.e. $(P, L)=\left(P_{\delta}, L_{\delta}\right)$. One has a canonical eigenspace decomposition $H^{0}\left(P_{\delta}, L_{\delta}\right)=\bigoplus_{x \in X \cap \delta} k \xi^{x}$, and so $\theta=\sum e_{x} \xi^{x}$.

Lemma 2.6.1. $\Theta$ does not contain any T-orbits entirely $\Leftrightarrow e_{x} \neq 0$ for the vertices of $\delta$.

Proof. It suffices to consider only 0-dimensional $T$-orbits, i.e. the fixed points of the $T$-action. These fixed points correspond in a 1-to-1 way to the vertices $x_{i} \in X \cap \delta$. Say $x_{0}$ is one of them. Then the section $\xi^{x_{0}}$ is the only one among $\xi^{x}$ which does not vanish at that point. Therefore, one must have $e_{x_{0}} \neq 0$.

Definition 2.6.2. $C(\Theta)=\left\{x \in X \mid e_{x} \neq 0\right\}$.

Corollary 2.6.3. $\Theta$ does not contain any T-orbits entirely $\Leftrightarrow \operatorname{Vert} \delta \subset$ $C(\Theta) \Leftrightarrow \delta=\operatorname{Conv} C(\Theta)$.

Definition 2.6.4. More generally, say $(P, L)=(P, L)[\Delta, \tau]$. Then for each $\theta \in R[\Delta, \tau]_{1}$ and for each $i$ we can consider its restriction on $R_{i}=k\left[S_{i}\right]$, and the set $C(\Theta)=\left\{x \in \rho^{-1} X \mid e_{x} \neq 0\right\}$ is well-defined.

Corollary 2.6.5. If $(P, L)=(P, L)[\Delta, \tau]$ then $\Theta$ does not contain any T-orbits entirely $\Leftrightarrow$ Vert $\Delta \subset C(\Theta)$. 
Definition 2.6.6. For a complex $\Delta$ of lattice polytopes referenced by a lattice $X, \operatorname{MP}^{\mathrm{fr}}[\Delta, C](k)$ will denote the groupoid of stable toric pairs $(P, \Theta)$ with a linearized line bundle $L=\mathcal{O}(\Theta)$ over $k=\bar{k}$ in which the arrows are the isomorphisms identical on $T$. As before, $\operatorname{MP}[\Delta, C](k)$ is a similar category in which we allow the isomorphisms which are not necessarily identities on $T$. Further, for a pointed complex $(\Delta, C)$ of lattice polytopes referenced by a lattice $X$ and with Vert $\Delta \subset C, \operatorname{MP}^{\mathrm{fr}}[\Delta, C](k)$ and $\operatorname{MP}[\Delta, C](k)$ will denote the groupoids of pairs $(P, \Theta)$ with $C(\Theta)=C$. Clearly,

$$
\operatorname{MP}[\Delta](k)=\coprod_{C, \text { Vert } \Delta \subset C} \operatorname{MP}[\Delta, C](k) .
$$

LEMma 2.6.7. If $\Delta$ consists of the lattice polytope $\delta_{i}$ and its faces then

$$
\begin{aligned}
\operatorname{MP}^{\mathrm{fr}}[\Delta](k) & \sim\left[\widehat{\operatorname{Fun}}_{\geq 0, i} / \mathbb{T}_{i}\right], \\
\operatorname{MP}[\Delta](k) & \sim\left[\widehat{\operatorname{Fun}}_{\geq 0, i} / \mathbb{T}_{i} \ltimes \operatorname{Sym} \delta\right]
\end{aligned}
$$

(see subsection 2.2 for definitions of these groups). If, in addition, a set $C \supset$ Vert $\delta$ is chosen then

$$
\begin{aligned}
\operatorname{MP}^{\mathrm{fr}}[\Delta, C](k) & \sim\left[\widehat{\operatorname{Fun}}_{i} / \mathbb{T}_{i}\right] \sim\left[\widehat{\mathbb{L}}_{i} / \widehat{\mathbb{K}}_{i}\right], \\
\operatorname{MP}[\Delta, C](k) & \sim\left[\widehat{\operatorname{Fun}}_{i} / \mathbb{T}_{i} \ltimes \operatorname{Sym} \delta\right] \sim\left[\widehat{\mathbb{L}}_{i} / \widehat{\mathbb{K}}_{i} \ltimes \operatorname{Sym} \delta\right]
\end{aligned}
$$

with $\widehat{\mathbb{K}}_{i}$ acting trivially.

Proof. All we have to do is to divide the set of the admissible $\theta$ 's by the equivalent relation induced by the choice of the origin in the torsor $\mathbf{L}_{i}^{-1}$. The latter is given by the action of the group $\mathbb{T}_{i}$.

THEOREM 2.6.8.

$$
\begin{aligned}
\operatorname{MP}^{\mathrm{fr}}[\Delta](k) & \sim\left[Z^{1}\left(\widehat{\mathbb{M}}_{\geq 0}^{*}[\Delta, \text { Vert } \Delta]\right) / \widehat{\mathbb{M}}^{0}[\Delta]\right] \quad \text { and } \\
\operatorname{MP}[\Delta](k) & \left.\sim\left[Z^{1}\left(\widehat{\mathbb{M}}_{\geq 0}^{*}[\Delta, \operatorname{Vert} \Delta]\right) / \widehat{\mathbb{M}}^{0}[\Delta] \ltimes \operatorname{Sym} \Delta\right]\right] .
\end{aligned}
$$

For a pointed complex $(\Delta, C)$ with Vert $\Delta \subset C$,

$$
\begin{aligned}
\operatorname{MP}^{\mathrm{fr}}[\Delta, C](k) & \sim\left[Z^{1}\left(\widehat{\mathbb{M}}^{*}[\Delta, C]\right) / \widehat{\mathbb{M}}^{0}[\Delta]\right] \\
& \sim\left[H^{1}\left(\widehat{\mathbb{M}}^{*}[\Delta, C]\right) / H^{0}\left(\widehat{\mathbb{M}}^{*}[\Delta, C]\right)\right] \\
\operatorname{MP}[\Delta, C](k) & \left.\sim\left[Z^{1}\left(\widehat{\mathbb{M}}^{*}[\Delta, C]\right) / \widehat{\mathbb{M}}^{0}[\Delta] \ltimes \operatorname{Sym}(\Delta, C)\right]\right] \\
& \left.\sim\left[H^{1}\left(\widehat{\mathbb{M}}^{*}[\Delta, C]\right) / H^{0}\left(\widehat{\mathbb{M}}^{*}[\Delta, C]\right) \ltimes \operatorname{Sym}(\Delta, C)\right]\right]
\end{aligned}
$$

with $H^{0}\left(\widehat{\mathbb{M}}^{*}[\Delta, C]\right)=H^{0}(\widehat{\mathbb{K}}[\Delta, C])$ acting trivially. For every element $l \in H^{0}(\Delta, \widehat{\mathbb{L}}[\Delta, C])$ (which describes a collection of pairs $\left.\left(P_{i}, \Theta_{i}\right)\right)$ whose image under the obstruction map

$$
H^{0}(\Delta, \underline{\mathbb{\mathbb { L }}}[\Delta, C]) \rightarrow H^{2}(\Delta, \underline{\widehat{\mathbb{K}}}[\Delta, C])
$$


is zero, there is an associated finite set of stable toric pairs and this set is a torsor over the group $H^{1}(\Delta, \widehat{\mathbb{K}}[\Delta, C])$. For each of these pairs

$$
\operatorname{Aut}(P, \Theta)=H^{0}\left(\widehat{\mathbb{M}}^{*}[\Delta, C]\right)=H^{0}(\Delta, \underline{\widehat{\mathbb{K}}}[\Delta, C])
$$

is a finite group.

Proof. A marked framed polarized $\operatorname{STV}(P, L)[\Delta, \tau]$ does not have any automorphisms. By Theorem 2.5.1 a choice of $\theta$ is equivalent to a choice of an element of $Z^{1}\left(\widehat{\mathbb{M}}^{*}[\Delta, C]\right)$. To describe the above groupoids we have to divide this set by the equivalence relation induced by choosing a different marking. The second part of the theorem is an application of Lemma 2.2.7.

2.7. The moment map for stable toric pairs over $\mathbb{C}$. Starting with a stable toric pair $(P, \Theta)$ over $k=\mathbb{C}$, we are going to define a map $P(\mathbb{C}) \rightarrow|\Delta|$ generalizing the usual moment map for toric varieties; see [Oda] for example. This construction further shows how well the cell decomposition $\Delta$ describes the essential properties of a stable toric pair.

Definition 2.7.1. Let $\theta=\sum_{x \in C(\Theta)} \xi_{x} \in H^{0}(L)$ be an equation of $\Theta$. The eigenfunctions $\xi_{x}$ are sections of the line bundle $L$ and at every point at least one of these functions is not zero.

The moment map $P \rightarrow|\Delta|$ is defined by the formula

$$
p \mapsto \frac{\sum\left|\xi_{x}(p)\right|^{2} x}{\sum\left|\xi_{x}(p)\right|^{2}} \in X_{\mathbb{R}}
$$

Let $P_{i}$ be an irreducible component of $P$ corresponding to a polytope $\delta_{i}$. The sections $\xi_{x}$ with $x \notin \delta$ are identically zero on $P_{i}$. Therefore, restriction of the above map to $P_{i}$ is a usual moment map for a projective toric variety. Its image is precisely the polytope $\delta$.

Since stable toric varieties are "built" of ordinary projective toric varieties, the next result easily follows from the description of the moment map for toric varieties.

TheOREM 2.7.2. 1. The moment map defines a continuous map between the topological spaces $P(\mathbb{C})$ and $|\Delta|$. The fiber over a point in the interior of a q-dimensional cell $\delta$ is $S^{q}=U(1)^{q}$.

2. Choose a set of representatives for $\xi_{x}$ 's (they are defined up to a common complex constant). Then the restriction of the moment map $P \rightarrow|\Delta|$ to the subset $P\left(\mathbb{R}_{\geq 0}\right)$ of points with nonnegative and real $\xi_{x}$ is a homeomorphism. 
Definition 2.7.3. By analogy with the usual terminology for toric varieties, we can call the $C^{\infty}$-varieties locally isomorphic to $P[\Delta,|\tau|]\left(\mathbb{R}_{\geq 0}\right)$ (i.e. locally isomorphic to $P[\Omega,|t|]\left(\mathbb{R}_{\geq 0}\right)$ varieties with sharp corners.

2.8. One-parameter families of stable toric pairs. Let $(\mathcal{R}, \mathfrak{m})$ be a DVR with the fraction field $\mathcal{K}, k$ be the residue field $\mathcal{R} / \mathfrak{m}, \eta=\operatorname{Spec} \mathcal{K}$ and $O=$ Spec $k$ be the generic and the special points of Spec $\mathcal{R}$ respectively. We will denote $\mathcal{S}=$ Spec $\mathcal{R}$. We will also denote by $s$ the uniformizing parameter, i.e., the generator of the ideal $\mathfrak{m}$.

TheORem 2.8.1. Let $T_{\eta}$ be a torus over $\mathcal{K}$ and $\left(P_{\eta}, \Theta_{\eta}\right)$ be a family over $\mathcal{K}$ of stable toric pairs with the linearized $T_{\eta^{-}}$action on $L_{\eta}=\mathcal{O}_{P_{\eta}}\left(\Theta_{\eta}\right)$. Then after a finite, possibly ramified cover this family can be completed to a family of stable toric pairs $(P, \Theta)$ over $\mathcal{R}$, and such a completion is unique up to an isomorphism.

Reduction Step 1. After an étale base change we can assume that the torus $T_{\eta}$ is split with the character group $X \stackrel{\sim}{\rightarrow} \mathbb{Z}^{r}$. We will continue to use the same letters $\mathcal{R}, \mathcal{K}$ etc. in order not to crowd the notation. The $T_{\eta}$ and the $\mathbb{G}_{m, \eta}$-action on $L_{\eta}$ give the action of a split torus $\mathbb{T}_{\eta}$ with the character group $\mathbb{X}=\mathbb{Z} \oplus X$. Now $T=\operatorname{Spec} \mathcal{R}[X]$ and $\mathbb{T}=\operatorname{Spec} \mathcal{R}[\mathbb{X}]$ will denote the corresponding split tori over $\mathcal{R}$.

We will start the proof of the theorem with the case when $P_{\eta}$ is geometrically irreducible, i.e. when the pair $\left(P_{\eta}, L_{\eta}\right) \otimes \overline{\mathcal{K}}$ corresponds to a single lattice polytope $Q \subset X_{\mathbb{R}}$.

Reduction Step 2. After another étale base change we can assume that $P_{\eta}$ admits a $\mathcal{K}$-point in the "interior", i.e., a point that over the algebraic closure $\overline{\mathcal{K}}$ induces a point in the main torus orbit.

Consider the $K$-algebra $R_{\eta}=\bigoplus_{d \geq 0} H^{0}\left(P_{\eta}, L_{\eta}^{d}\right)$. The $\mathcal{K}$-point gives an embedding $T_{\eta} \rightarrow P_{\eta}$ and, consequently, the embedding $R_{\eta} \subset \mathcal{K}[\mathbb{X}]$, which allows us to fix an isomorphism $R_{\eta} \simeq \mathcal{K}[$ Cone $Q]$. We have an element $\theta_{\eta} \in$ $H^{0}\left(P_{\eta}, L_{\eta}\right)$ which can be uniquely written as

$$
\theta_{\eta}=\sum \xi_{\eta, x}=\sum_{x \in X \cap Q} c(x) \zeta^{(1, x)}, \quad c(x) \in \mathcal{K} .
$$

As before, let $C=\{x \mid c(x) \neq 0\}$. The discrete valuation on $\mathcal{K}$ defines an integral-valued function on $C: \psi: x \mapsto \operatorname{val} c(x)$, and now

$$
c(x)=c^{\prime}(x) \cdot s^{\psi(x)} \quad \text { with } \operatorname{val} c^{\prime}(x)=0 .
$$

We will denote by $c_{0}(x)$ the residue of $c^{\prime}(x)$ in $k$. 
Definition 2.8.2. Consider the convex hull $\mathrm{ConvHull}_{\psi}$ in $X_{\mathbb{R}} \oplus \mathbb{R}$ of the rays

$$
\{(x, h) \mid x \in C, h \geq \psi(x)\} .
$$

The graph of the lower envelope of this convex hull is a piecewise-linear function $g_{\psi}: Q \rightarrow \mathbb{R}$. Thinking of $X$ here as the subset $(1, X)$ of $\mathbb{X}$ of degree 1 , we can extend this function to a function on Cone $Q$ by dilations: $g_{\psi}(\chi)=d g_{\psi}(\chi / d)$, where $d=\operatorname{deg} \chi$. The fields of linearity of $g_{\psi}$ define a cell decomposition $\Delta$ of the polytope $Q$. For each cell $\delta_{i}$ in this decomposition let $C_{i}$ be the subset of $X \cap \delta_{i}$ of the points with $\psi(1, x)=g_{\psi}(1, x)$, i.e. the points $x$ for which the corresponding point in $\mathbb{X} \oplus \mathbb{Z}$ lies on the lower boundary of ConvHull . $_{\text {. }}$

A pointed decomposition $\left(\Delta_{0}, C_{0}\right)=\left\{\left(\delta_{i}, C_{i}\right)\right\}$ defined by the above lifting property is usually called regular or coherent, see [GKZ, Ch. 7].

Definition 2.8.3. Define the $\mathcal{R}$-subalgebra $R \subset \mathcal{K}[\mathbb{X}]$ as the $\mathcal{R}$-module with the generators $\left\{s^{h} \zeta^{\chi} \mid \chi \in\right.$ Cone $\left.Q, h \geq g_{\psi}(\chi)\right\}$. Although $R$ is not a semigroup algebra, it formally corresponds to the semigroup of integral elements in $\mathbb{Z} \oplus \mathbb{X}$ lying inside the cone over $\mathrm{ConvHull}_{\psi}$. Since this is a rational finitely generated cone, the algebra $R$ is finitely generated over $\mathcal{R}$.

Now we wish to look at the residue algebra $R_{0}=R / \mathfrak{m} R$.

Lemma 2.8.4. The algebra $R_{0}$ is nilpotent-free if and only if the function $g_{\psi}$ is integral-valued on $\mathbb{X} \cap$ Cone $Q$. In this case, $R_{0}$ is isomorphic to the standard algebra $R[\Delta, 1]$ over the field $k$.

Proof. For each $\chi \in \mathbb{X} \cap$ Cone $Q$ the corresponding eigenspace in $R_{0}$ is a 1-dimensional $k$-space with the generator $\bar{\zeta}_{\chi}$ which is the residue of $s\left\ulcorner g_{\psi}(\chi)\right\urcorner \zeta^{\chi}$ ( \ulcorner\urcorner denotes the upper integral part). It is clear that for the points $(1, x)$ corresponding to the edges of the lower envelope, $g_{\psi}(\chi)$ is integral, but in general this may not be true. If for some $x$ the value $g_{\psi}(\chi)$ is not integral then a power of $\bar{\zeta} \chi$ corresponds to an element lying in the interior of $\mathrm{ConvHull}_{\psi}$ that can be represented as the sum of $\bar{\zeta}$ 's for the edges plus $(0, n)$ with $n>0$. Thus, in the residue algebra this power is zero, and we have a nilpotent element.

Now assume that all the points on the lower envelope of the cone over $\mathrm{ConvHull}_{\psi}$ are integral and consider the product $\bar{\zeta}_{\chi_{1}} \bar{\zeta}_{\chi_{2}}$. Geometrically, it corresponds to the sum of the corresponding two elements on the lower envelope of Cone $\left(\mathrm{ConvHull} \mathrm{l}_{\psi}\right)$. If $\chi_{i}$ belong to the same cone, we get a point on the same face of the envelope, and so $\bar{\zeta}_{\chi_{1}} \bar{\zeta}_{\chi_{2}}=\bar{\zeta}_{\chi_{1}+\chi_{2}}$. Otherwise, we get a point in the interior of Cone $\left(\mathrm{ConvHull}_{\psi}\right)$ which corresponds to an element on the lower envelope plus $(0, n)$ with $n>0$. In this case, $\bar{\zeta}_{\chi_{1}} \bar{\zeta}_{\chi_{2}}=0$. This is precisely the description of the algebra $R_{0}[\Delta, 1]$ in Section 2.3. By the results of section 2.3 the algebra $R_{0}$ in this case is nilpotent-free. 
Reduction Step 3 . The condition that $g_{\psi}$ is integral-valued on $\mathbb{X} \cap$ Cone $Q$ can be achieved by a ramified base change $s=t^{n}$. Make this base change.

We are now ready to complete the proof of Theorem 2.8.1 in the case of geometrically irreducible $P_{\eta}$. Simply consider $P=\operatorname{Proj}_{\mathcal{R}} R$ for the abovedefined algebra $R$. This algebra is a free module over $\mathcal{R}$; hence $P$ is flat over Spec $\mathcal{R}$. We have established that the central fiber is an STV. The line bundle $L=\mathcal{O}(1)$ comes with a global section $\theta=\sum c(x) \zeta^{(1, x)}$. By the construction, on the central fiber it restricts to a section of $H^{0}\left(P_{0}, \mathcal{O}(1)\right)$ with the equation

$$
\theta_{0}=\sum_{x \in C} c_{0} \bar{\zeta}^{(1, x)}
$$

It does not contain 0-dimensional toric orbits since for each vertex of a polytope $\delta_{i}$ the corresponding coefficient $c_{0}(x)$ is nonzero. This proves the existence.

2.8.5. To summarize, if the generic fiber is geometrically irreducible then the central fiber $\left(P_{0}, \Theta_{0}\right)$ corresponds to the following data:

1. The decomposition $\left(\Delta_{0}, C_{0}\right)$ defined by the integral-valued function $\psi$,

2. The trivial 1-cohomology class $\tau_{0}=1 \in Z^{1}\left(\Delta_{0}, \underline{\widehat{\mathbb{X}}}\right)$,

3. The element $\hat{f}_{0}=\left(c_{0}(x)\right) \in C_{0}\left(\Delta_{0}, \widehat{\widehat{\operatorname{Fun}}}\left[\Delta_{0}, C_{0}\right]\right)$.

The pair $\left(\tau_{0}, \hat{f}_{0}\right)$ is an element of the group $Z^{1}\left(\widehat{\mathbb{M}}^{*}\left[\Delta_{0}, C_{0}\right]\right)$ as defined earlier.

Let us prove the uniqueness of the pointed cell decomposition for the central fiber $\left(P_{0}, L_{0}\right)$. Let $f:(P, \Theta) \rightarrow$ Spec $R$ be an extended flat family. As above, after an additional base change (which does not change the type of the central fiber) we can make the tori split and put the generic fiber in the standard form. By the results of Section 2.5 on each geometric fiber the higher cohomologies of $L^{d}, d>0$, vanish. Therefore, by cohomology and base change the $\mathcal{O}_{\mathcal{S}}$-algebra $f_{*} L^{d}$ is locally free. The $\mathbb{T}$-action gives a canonical splitting of this algebra into the direct sum of $\mathbb{X}$-eigenspaces which therefore also have to be locally free and hence invertible. Let $\theta=\sum \xi_{x} \in R$ be the equation of the divisor $\Theta$. For each $f=\left(n_{x}\right) \in \operatorname{Fun}\left(C, \mathbb{N}_{\geq 0}\right)$ define $\xi^{f}=\prod \xi_{x}^{n_{x}}$.

Our condition on the divisor $\Theta_{0}$ implies that the algebra $R$ is finite over the subalgebra ${ }_{1} R$ generated by $\xi^{f}$ 's, in fact for every $\mathbb{X}$-homogeneous element $r \in R$ we have $r^{n}=$ unit $\cdot \xi^{f}$ for some $n>0$. The cell decomposition $\Delta$ depends only on whether for each pair of homogeneous elements $r_{1}, r_{2}$ the residue of $\left(r_{1} r_{2}\right)^{n}$ is zero or not, i.e. whether $\xi^{f_{1}+f_{2}}$ equals zero or not. On the other hand, by finiteness for every $\xi^{f}$ one has $\xi^{n f}=c(n f, g) \xi^{g}$ for some $g$ such that the residue of $\xi^{g}$ is not zero. Finally, the element $c(n f, g) \in \mathcal{K}$ can be computed on the generic fiber as $\xi_{\eta}^{n f} / \xi_{\eta}^{g}$. This proves the uniqueness of the 
decomposition. It also shows the uniqueness of the $\mathcal{O}_{S}$-subalgebra $\oplus f_{*} L^{d}$ of $\mathcal{K}[\mathbb{X}]$, and therefore of its Proj, i.e. the family $P$. Clearly, the divisor $\Theta$, which is the closure of $\Theta_{\eta}$, is unique as well.

In the general case, the pair $\left(P_{\eta}, \Theta_{\eta}\right) \otimes \overline{\mathcal{K}}$ corresponds to a complex $(\Delta, C)=\left\{\left(Q_{j}, C_{j}\right)\right\}$. After the étale base change, we represent the algebra $R_{\eta}$ in the standard form $R_{\eta}[\Delta, \tau]$, with $\tau \in \operatorname{Hom}\left(C_{1}(\underline{\mathbb{X}}), \mathcal{K}^{*}\right)$ and the equation $\theta$ as the system of equations $\theta_{j}$ which give an element $\hat{f} \in \operatorname{Hom}\left(C_{0}(\underline{\operatorname{Fun}}[\Delta, C]), \mathcal{K}\right)$. The pair $(\hat{f}, \tau)$ has to be an element of $Z^{1}\left(\widehat{\mathbb{M}}^{*}\right)$ as defined earlier.

For each of the algebras $R_{\eta, j}$ repeat the above procedure, defining a finitely generated $\mathcal{R}$-subalgebra $R_{j}$ in each. One then checks that the gluing functions between neighboring $\zeta_{j i}$ are units in $\mathcal{R}$. Therefore, the image of the collection $R_{j}$ in $R_{\eta}$ is an $\mathcal{R}$-algebra of the type $R\left[\Delta_{0}, C_{0}\right]$ for the pair $\left(\tau^{\prime}, \hat{f}^{\prime}\right) \in Z^{1}\left(\widehat{\mathbb{M}}^{*}\right)$ with the coefficients in $\mathcal{R}$, as opposed to simply in $\mathcal{K}$. The central fiber will then correspond to $\left(R_{0}, \theta_{0}\right)$ for the pair $\left(\tau_{0}, \hat{f}_{0}\right)$ which is the residue of $\left(\tau^{\prime}, \hat{f}^{\prime}\right)$ in $k$.

The uniqueness of the decomposition and the subalgebra $\oplus f_{*} L^{d}$ follows because by the special case we have the uniqueness of decomposition and the subalgebras for each $Q_{j}$. The theorem is now completely proved.

2.8.6. To summarize, the central fiber $\left(P_{0}, \Theta_{0}\right)$ corresponds to the following data:

1. The sub decomposition $\left(\Delta_{0}, C_{0}\right)$ of $(\Delta, C)$ defined by the integral-valued functions $\psi_{j}$,

2. The 1-cohomology class $\tau_{0} \in Z^{1}\left(\Delta_{0}, \underline{\widehat{\mathbb{X}}}\right)$ that is the residue of the image of the class $\tau^{\prime}$ under the natural morphism $Z^{1}(\Delta, \underline{\widehat{\mathbb{X}}}) \rightarrow Z^{1}\left(\Delta_{0}, \underline{\widehat{\mathbb{X}}}\right)$,

3. The element $\hat{f}_{0} \in C^{0}\left(\Delta_{0}, \widehat{\widehat{\operatorname{Fun}}}\left[\Delta_{0}, C_{0}\right]\right)$ which is the residue of the image of the element $\hat{f}^{\prime}$ under the natural morphism $C^{0}(\widehat{\underline{\mathrm{Fun}}}[\Delta, C]) \rightarrow$ $C^{0}\left(\underline{\underline{\text { Fun }}}\left[\Delta_{0}, C_{0}\right]\right)$.

The pair $\left(\tau_{0}, \hat{f}_{0}\right)$ is an element of the group $Z^{1}\left(\widehat{\mathbb{M}}^{*}\left[\Delta_{0}, C_{0}\right]\right)$ as was defined earlier.

2.9. The modified complex $\mathbb{M}_{*}$. Starting with a pointed complex $(\Delta, C)$ we are now going to define the "semigroup" analogs of the chain complexes $C_{*}(\underline{\mathbb{X}}), C_{*}(\underline{\text { Fun }})$ and $\mathbb{M}_{*}$. We will only need them in degrees 0,1 and 2.

Definition 2.9.1. We will define $S C_{0}\left(\underline{\mathbb{X}}_{\geq 0}\right)=\oplus \mathbb{X}_{i}$. The natural projections Cone $\delta_{i} \rightarrow$ Cone $|\Delta|$ extend by linearity to a map $\varrho: \oplus$ Cone $\delta_{i} \rightarrow$ Cone $|\Delta|$. In degree $n>0$ we will define $S C_{n}\left(\underline{\mathbb{X}}_{\geq 0}\right)$ as a free abelian semigroup on the generators $\left(\bar{\chi}_{0}, \chi_{1}, \ldots, \chi_{n}\right)$ with 


$$
\begin{gathered}
\bar{\chi}_{0} \in \oplus \text { Cone } \delta_{i}, \quad \chi_{1}, \ldots, \chi_{n} \in \cup \text { Cone } \delta_{i} \subset \oplus \text { Cone } \delta_{i} \\
\text { such that } \varrho \bar{\chi}_{0}=\varrho \chi_{1}=\ldots=\varrho \chi_{n},
\end{gathered}
$$

modulo a certain natural equivalence relation. We will not even spell it out in degrees $n \geq 2$ since we do not need it. In degree 1 it has the form

$$
\begin{gathered}
\left(\bar{\chi}_{1}+\bar{\chi}_{2}, \chi\right)=\left(\bar{\chi}_{1}, \chi_{1}\right)+\left(\bar{\chi}_{2}, \chi_{2}\right)+\left(\chi_{1}+\chi_{2}, \chi\right), \\
(\chi, \chi)=0
\end{gathered}
$$

which reduces to the linearity condition

$$
\left(\chi_{1}^{\prime}+\chi_{2}^{\prime}, \chi_{1}+\chi_{2}\right)=\left(\chi_{1}^{\prime}, \chi_{1}\right)+\left(\chi_{2}^{\prime}, \chi_{2}\right)
$$

when $\chi_{1}, \chi_{2}$ belong to the same cone.

We will define the differential $S C_{1}\left(\underline{\mathbb{X}}_{\geq 0}\right) \rightarrow S C_{0}\left(\underline{\mathbb{X}}_{\geq 0}\right)$ by $\left(\bar{\chi}_{0}, \chi_{1}\right) \mapsto$ $\bar{\chi}_{0}-\chi_{1}$. The image of the differential from $S C_{2}\left(\underline{\mathbb{X}}_{\geq 0}\right)$ to $S C_{1}\left(\underline{\mathbb{X}}_{\geq 0}\right)$ will be interpreted as an equivalence relation on $S C_{1}\left(\underline{\mathbb{X}}_{\geq 0}\right)$ generated by

$$
\left(\chi_{1}, \chi_{2}\right)+\left(\bar{\chi}_{0}, \chi_{1}\right)=\left(\bar{\chi}_{0}, \chi_{2}\right) .
$$

Hence, $S C_{1} / S B_{1}\left(\underline{\mathbb{X}}_{\geq 0}\right)$ will denote the quotient of the semigroup $S C_{1}\left(\underline{\mathbb{X}}_{\geq 0}\right)$ by this equivalence relation.

Remark 2.9.2. There is a natural ring homomorphism $k\left[S C_{1} / S B_{1}\left(\underline{\mathbb{X}}_{\geq 0}\right)\right] \rightarrow$ $k\left[C_{1} / B_{1}(\underline{\mathbb{X}})\right]$. It sends every $x^{\left(\sum \bar{\chi}_{i}, \chi\right)}$ with not all $\bar{\chi}_{i}$ belonging to a common cone to 0 . This defines an open embedding on the Spec's of these algebras in the opposite direction.

Definition 2.9.3. For the cosheaf Fun we define $S C_{0}\left(\underline{\text { Fun }}_{\geq 0}\right)=C_{0}\left(\underline{\text { Fun }}_{\geq 0}\right)$. $S C_{1}\left(\underline{\text { Fun }}_{\geq 0}\right)$ will be the free abelian group with the generators $\left(f_{0}, f_{1}\right)$ with $\varrho \phi f_{0}=\varrho \phi f_{1}$.

Definition 2.9.4. We define the complex $\mathrm{SM}_{*}$ as follows:

$$
\begin{aligned}
& \mathrm{SM}_{0}=S C_{0}\left(\underline{\mathbb{X}}_{\geq 0}\right), \quad \mathrm{SM}_{1}=S C_{0}\left(\underline{\mathrm{Fun}}_{\geq 0}\right) \oplus S C_{1}\left(\underline{\mathbb{X}}_{\geq 0}\right), \\
& \mathrm{SM}_{2}=S C_{1}\left(\underline{\mathrm{Fun}}_{\geq 0}\right) \oplus S C_{2}\left(\underline{\mathbb{X}}_{\geq 0}\right) .
\end{aligned}
$$

The differential $\mathrm{SM}_{1} \rightarrow \mathrm{SM}_{0}$ is defined as one should expect, $\left(f_{0},\left(\bar{\chi}_{0}, \chi_{1}\right)\right) \mapsto$ $\phi f_{0}+\bar{\chi}_{0}-\chi_{1}$. The image of the differential $\mathrm{SM}_{2} \rightarrow \mathrm{SM}_{1}$ again will be interpreted as an equivalence relation generated by

$$
f_{0}=f_{1}+\left(\phi f_{0}, \phi f_{1}\right) \text { and }\left(\chi_{1}, \chi_{2}\right)+\left(\bar{\chi}_{0}, \chi_{1}\right)=\left(\bar{\chi}_{0}, \chi_{2}\right) .
$$

LEMmA 2.9.5. The semigroups $S C_{1} / S B_{1}(\underline{\mathbb{X}})$ and $\mathrm{SM}_{1} / B_{1}\left(\mathrm{SM}_{*}\right)$ are finitely generated and finitely presented. 
Proof. We start with $S C_{1} / S B_{1}(\underline{\mathbb{X}})$. If $\chi_{1}, \chi_{2}$ are in the same Cone $\delta_{i}$ then the relations imply that $\left(\bar{\chi}_{1}+\bar{\chi}_{2}, \chi_{1}+\chi_{2}\right)=\left(\bar{\chi}_{1}, \chi_{1}\right)+\left(\bar{\chi}_{2}, \chi_{2}\right)$. Hence the symbols $(\bar{\chi}, \chi)$ for a fixed $\delta_{i}$ form a semigroup. This is the kernel of a homomorphism from a semigroup of integral elements in a finitely generated rational cone in the space $\left(\left(\oplus_{j}\right.\right.$ Cone $\left.\delta_{j}\right) \oplus$ Cone $\left.\delta_{i}\right) \otimes \mathbb{R}$ to $\mathbb{X}_{\mathbb{R}}$. Therefore, it is finitely generated. The semigroup $S C_{1} / S B_{1}(\underline{\mathbb{X}})$ is generated by these semigroups for different cells $\delta_{i}$, hence it is finitely generated as well.

Adding $C_{0}$ ( $\left.\underline{\text { Fun }}\right)$ together with our relations does not add more new generators than the number of generators in $H_{0}$ (Fun), which is definitely finite. Finally, by a standard result of the semigroup theory every finitely generated commutative semigroup is finitely presented.

Lemma 2.9.6. Let $H$ and $L$ be a finitely generated commutative semigroup and group respectively, and $f: H \rightarrow L$ a homomorphism defining an action on the scheme $V=\operatorname{Spec} H$ by the dual split group of multiplicative type $T=\operatorname{Spec} \mathbb{Z}[L]$. If the image of the invertible elements of $H$ is a subgroup of finite index in $L$ then the action morphism $T \times V \rightarrow V \times V$ is finite. In other words, in this case the T-action is proper with finite stabilizers. If the image of the invertible elements is all of $L$ then the $T$-action is free; i.e. $T \times V \rightarrow V \times V$ is a closed embedding.

Proof. The morphism $T \times V \rightarrow V \times V$ is defined by the semigroup homomorphism $H \times H \rightarrow L \times H,\left(h_{1}, h_{2}\right) \mapsto\left(f h_{1}, h_{1}+h_{2}\right)$. Take any $(l, h) \in L \times H$. If $n l=f h_{1}$ with $h_{1}$ invertible then $\left(h_{1}, n h-h_{1}\right) \mapsto n(l, h)$.

Corollary 2.9.7. The action of the torus $\operatorname{Spec} \mathbb{Z}\left[\mathrm{SM}_{0}\right]=\operatorname{Spec} \mathbb{Z}\left[C_{0}(\underline{\mathbb{X}})\right]$ on the scheme $\operatorname{Spec} \mathbb{Z}\left[\mathrm{SM}_{1} / B_{1}\left(\mathrm{SM}_{*}\right)\right]$ is proper with finite stabilizers.

Proof. Indeed, in this case already the image of the invertible elements of $C_{0}\left(\underline{\text { Fun }}_{\geq 0}\right)$ in $C_{0}(\underline{\mathbb{X}})$ has finite index.

Definition 2.9.8. For each $\Delta^{\prime} \geq \Delta$ the ideal $I\left[\Delta^{\prime}\right] \subset \mathbb{Z}\left[\mathrm{SM}_{1} / B_{1}\left(\mathrm{SM}_{*}\right)\right]$ is generated by $(\bar{\chi}, \chi)=\left(\sum \chi_{i}, \chi\right)$ such that there is no common polytope $\delta$ so that all $\chi_{i}, \chi \in$ Cone $\delta$. Similarly, for each $\left(\Delta^{\prime}, C^{\prime}\right) \geq(\Delta, C)$ we will denote by $I\left[\Delta^{\prime}, C^{\prime}\right]$ the ideal generated by these elements plus $f_{x, i} \in \operatorname{Fun}_{i}$ for all $x \in X \cap \delta \backslash C_{i}$.

\subsection{Moduli of stable toric pairs.}

Lemma 2.10.1. Let $f:(P, \Theta) \rightarrow S$ be a flat family of stable toric pairs with a linearized action of a split torus $T / S$ over a connected locally Noetherian base $S$. If one geometric fiber $\left(P_{s}, \Theta_{s}\right)$ corresponds to a cell decomposition of a polytope $Q \subset \mathbb{R}$ then any other geometric fiber corresponds to a cell decomposition of the same polytope $Q$. 
Proof. Since on each geometric fiber the higher cohomologies of $L^{d}, d>0$, vanish, by cohomology and base change the sheaves $f_{*} L^{d}$ are locally free. The action by $\mathbb{T}=\mathbb{G}_{m} \oplus T$ defines a decomposition of $\oplus_{d \geq 0} f_{*} L^{d}$ into a direct sum of eigenspaces over all $\chi \in \mathbb{X}$. They also must be locally free, and since $S$ is connected - of a constant rank. By looking at the fiber $\left(P_{s}, \Theta_{s}\right)$ we see that each of these eigenspaces is 1-dimensional if $\chi \in \operatorname{Cone} Q$ and 0 -dimensional otherwise. Therefore, the same is true for any other fiber, and this property defines the type of the fiber completely.

Definition 2.10.2. We will refer to the above as the convex 1-sheeted case.

Remark 2.10.3. For the purposes of moduli in this paper we will restrict ourselves to this case only (and its analogs when the action on $L$ is not linearized or when there is an abelian part). We call that a general linearized stable toric pair corresponds to a complex $\rho:|\Delta| \rightarrow X_{\mathbb{R}}$ which is quite general: the image in $X_{\mathbb{R}}$ need not be convex and the map can have several sheets. The moduli of such pairs are more complicated and will be left for another occasion.

Definition 2.10.4. Fix a lattice $X$ and the corresponding split torus $T=\operatorname{Spec} \mathbb{Z}[X]$. Denote by $\mathcal{T P}^{\text {fr }}$ the moduli stack on the category of locally Noetherian schemes associating to each scheme $S$ the groupoid of flat families $(P, \Theta) / S$ together with a linearized action of $T_{S}$. This groupoid is equivalent to the groupoid of flat families $(P, L, \theta)$ of linearized polarized toric varieties together with a section $\theta \in \Gamma\left(S, f_{*} L\right)$.

By the above, for a fixed polytope $Q \subset X_{\mathbb{R}}$ we have a connected substack $\mathcal{T} \mathcal{P}^{\text {fr }}[Q]$ for which every geometric fiber in a family corresponds to a cell decomposition of $Q$.

Definition 2.10.5. For a pointed cell decomposition $(\Delta, C)$ let $\mathcal{T} \mathcal{P}^{\mathrm{fr}}[\Delta, C]$ be a substack in which for each geometric fiber the corresponding cell decomposition is $\geq(\Delta, C)$.

Lemma 2.10.6. $\quad \mathcal{T P}^{\mathrm{fr}}[\Delta, C]$ is open in $\mathcal{T} \mathcal{P}^{\mathrm{fr}}[Q]$.

Proof. For a geometric fiber the condition that the cell decomposition is $\geq(\Delta, C)$ is the condition that for the generators of eigenspaces $R_{\chi_{i}}$ with $\chi^{\prime}$ s in the same Cone $\delta\left(R=\oplus_{d \geq 0} f_{*} L^{d}\right)$ the products are not zero and that residues of homogeneous components of $\theta$ for all $x \in C$ are not zero. These all are open conditions.

Lemma 2.10.7. The union of all $\mathcal{T} \mathcal{P}^{\mathrm{fr}}[\Delta, C]$ covers $\mathcal{T} \mathcal{P}^{\mathrm{fr}}[Q]$.

Proof. This is obvious since every fiber induces some $(\Delta, C)$. 
TheOREM 2.10.8. $\quad \mathcal{T P}^{\mathrm{fr}}[\Delta, C]$ is a separated Artin stack of finite presentation over $\mathbb{Z}$ with finite stabilizers. It has a coarse moduli space $\operatorname{TP}^{\mathrm{fr}}[\Delta, C]$ which is a separated scheme over $\mathbb{Z}$.

Proof. We claim that $\mathcal{T} \mathcal{P}^{\mathrm{fr}}[\Delta, C]$ coincides with the quotient stack

$$
\left[\mathrm{Spec} \mathbb{Z}\left[\mathrm{SM}_{1} / B_{1}\left(\mathrm{SM}_{*}\right)\right] / \operatorname{Spec} \mathbb{Z}\left[\mathrm{SM}_{0}\right]\right]
$$

for the action defined in the previous subsection where we checked that the stabilizer of the action is finite. The existence of a coarse moduli space for such a stack as a separated algebraic space is the main result of [KM].

Indeed, let $(P, \Theta) / S$ be any flat family of stable toric pairs. First of all, a family like this is equivalent to a family $(P, L, \theta), \theta \in H^{0}(P, L)$ satisfying the necessary conditions on geometric fibers. Secondly, we claim that it is equivalent to giving a locally free graded $\mathcal{O}_{S^{-}}$algebra $(R, \theta)$ with an element of degree 1, again with appropriate conditions on geometric fibers. Given $(P, L, \theta)$, we take $R=\oplus_{d \geq 0} f_{*} L^{d}$. Vice versa, given $R$, the pair $(P, L)$ is recovered as (Proj $R, \mathcal{O}(1))$. Hence, the moduli of our pairs are essentially the moduli of algebras $(R, \theta)$.

In an étale neighborhood $\operatorname{Spec} D \rightarrow S$ of any point locally in étale topology we can find the generators in the subalgebras $R_{i} \subset R=\oplus_{d \geq 0} f_{*} L^{d}$ corresponding to each cell $\delta_{i} \in \Delta$ and identify the $D$-algebra $R$ with the union of semigroup algebras $R_{i}$. One sees that the relation among these elements is precisely a homomorphism from the semigroup algebra $\mathbb{Z}\left[S C_{1} / S B_{1}(\underline{\mathbb{X}})\right]$ to $D$, and that the pair $(R, \theta)$ uniquely defines a homomorphism from the semigroup algebra $\mathbb{Z}\left[\mathrm{SM}_{1} / B_{1}\left(\mathrm{SM}_{*}\right)\right]$ to $D$. Hence, locally we get a $D$-point of the scheme Spec $\mathbb{Z}\left[\mathrm{SM}_{1} / B_{1}\left(\mathrm{SM}_{*}\right)\right]$. To get the moduli stack $\mathcal{T} \mathcal{P}^{\text {fr }}[\Delta, C]$ we have to divide this scheme by the choices made, and that amounts to the torus action by $\operatorname{Spec} \mathbb{Z}\left[\mathrm{SM}_{0}\right]$.

By $[\mathrm{KM}]$ there is a coarse moduli space as an algebraic space. However, for a proper torus action with finite stabilizers the quotient of an affine scheme is simply the affine scheme corresponding to the subring of invariants. Therefore, in the case at hand it is the scheme corresponding to the semigroup algebra $\mathbb{Z}[K[\Delta, C]]$, where $K=\operatorname{ker}\left(\mathrm{SM}_{1} / B_{1}\left(\mathrm{SM}_{*}\right) \rightarrow \mathrm{SM}_{0}\right)$.

Remark 2.10.9. Note the role of the ideals $I\left[\Delta^{\prime}\right]$ and $I\left[\Delta^{\prime}, C\right]$. The first one defines a closed subscheme over which the fibers in our family are varieties of type $\leq \Delta^{\prime}$ and $\geq \Delta$. The second defines a subscheme over which the pair $(P, \Theta)$ have the type $\leq\left(\Delta^{\prime}, C^{\prime}\right)$ and $\geq(\Delta, C)$.

THEOREM 2.10.10. $\quad \mathcal{T}^{\mathrm{fr}}[Q]$ is a proper Artin stack of finite presentation over $\mathbb{Z}$ with finite stabilizers. It has a coarse moduli space $\operatorname{TP}^{\mathrm{fr}}[Q]$ which is a proper scheme over $\mathbb{Z}$. 
Proof. By the result of Section 2.8, any 1-parameter family over a complete discrete valuation field $\mathcal{K}$ can be completed to a family over the DVR after a finite base change. Therefore, $\mathcal{T} \mathcal{P}^{\text {fr }}[Q]$ is complete. Moreover, the decomposition $\left(\Delta_{0}, C_{0}\right)$ for the central fiber is uniquely defined, so that the limit is contained in a unique minimal $\mathcal{T} \mathcal{P}^{\mathrm{fr}}\left[\Delta_{0}, C_{0}\right]$. Since the latter is separated, $\mathcal{T P}^{\mathrm{fr}}[Q]$ is separated. The coarse moduli space is obtained by gluing the coarse moduli spaces of all the $\mathcal{T P}^{\mathrm{fr}}[\Delta, C]$ 's.

2.11. Approximation of the moduli space. Here, we are going to show that the just constructed moduli space $M=\operatorname{TP}^{\mathrm{fr}}[Q]$ admits a finite morphism to an easily describable projective scheme which can be considered to be its simplification. Consequently, $\operatorname{TP}^{\mathrm{fr}}[Q]$ is itself projective. Moreover, both schemes admit toric stratifications in which every stratum in one scheme corresponds in a 1-to-1 way to an isogenic stratum in the second. We may say that our moduli space and its simplification differ in only finitely many ways.

Definition 2.11.1. Denote $C_{\max }=X \cap Q$ and let $\phi$ be as before the natural semigroup homomorphism from $\operatorname{Fun}\left(C_{\max }, \mathbb{Z}_{+}\right)$to Cone $Q \subset \mathbb{X}$ sending $1_{x}$ to $(1, x)$. Take a rational point $q \in(1, Q)$ and let $N$ be the minimal natural number with $N q$ integral. Let $H_{q}$ be the semigroup $\phi^{-1}\left(\mathbb{Z}_{+} N q\right)$.

Definition 2.11.2. On the other hand, consider the map Fun $\left(C_{\max }, \mathbb{R}_{+}\right) \cap$ $\left\{\left(f_{x}\right) \mid \sum f_{x}=1\right\}$ to $(1, Q)$. This is a map of polytopes: from the simplex on the vertices $1_{x}$ of dimension $\# C_{\max }-1$, which we will denote $\sigma$ to $Q$. Let $\sigma_{q}$ be the fiber of this polytopal map over $q$.

It is clear that $H_{q}$ is a saturated sub-semigroup in $\operatorname{Fun}\left(C_{\max }, \mathbb{Z}\right)$ consisting of all integral points in the cone over $\sigma_{q}$.

Lemma 2.11.3. Faces of $\sigma_{q}$ are in a one-to-one correspondence with lattice subpolytopes $\delta$ of $Q$ containing $q$ in their interior. Vertices correspond to simplices with this property. If $\delta=\left\langle x_{0}, \ldots, x_{r}\right\rangle$ is such a simplex and $(1, q)=\sum c_{i}\left(1, x_{i}\right)$ then the coordinates of the corresponding vertex are $\left(c_{i}\right)$.

Proof. This is truly obvious.

Definition 2.11.4. $F_{q}=\operatorname{Proj} H_{q}$.

2.11.5. So, $F_{q}$ is a projective normal toric scheme corresponding to the lattice polytope obtained by a dilation of $\sigma_{q}$ (we are not interested in the ample linearized sheaf on $F_{q}$, only in the scheme itself). If two fibers $\sigma_{q_{1}}, \sigma_{q_{2}}$ are combinatorially equivalent and have parallel faces then $F_{q_{1}}=F_{q_{2}}$ - since the dual fans are the same. If $\sigma_{q_{2}}$ is obtained by "degenerating" $\sigma_{q_{1}}$, i.e. by contracting some of the faces, then there is a natural morphism $F_{q_{1}} \rightarrow F_{q_{2}}$; this corresponds to a natural map of fans. It is birational if $\sigma_{q_{i}}$ have the same dimension, for example if both $q_{i}$ lie in the interior of $Q$. 
The polytope $Q$ is naturally subdivided into locally closed polytopal domains $D_{\alpha}$ such that for $q$ 's in the same $D_{\alpha}$ the fibers are equivalent, and for $D_{\alpha_{2}} \subset \overline{D_{\alpha_{1}}}$, fibers $\sigma_{q_{2}}$ are degenerations of fibers $\sigma_{q_{1}}$. This stratification is defined by intersecting the projections of faces of $\sigma$. Indeed, for two points in $D_{\alpha}$ the cells $\delta$ containing these points in their interior are exactly the same.

Fix one scheme $F_{\alpha}$ for every stratum $D_{\alpha}$. We have obtained a partially ordered set $J=\alpha$, ordered by the "degeneration relation" and a system of projective schemes $F_{\alpha}$ indexed by $J$ with morphisms $F_{\alpha_{1}} \rightarrow F_{\alpha_{2}}$ for each $\alpha_{1} \geq \alpha_{2}$.

Definition 2.11.6. Define $M_{\text {simp }}=\lim _{J} F_{\alpha}$.

Remark 2.11.7. Note that in the category of schemes over a fixed base inverse limits over any finite category $J$ exist. Indeed, by a standard result from category theory it would suffice for finite products and equalizers to exist, and both are particular cases of fiber products. It is also easy to show (although we do not need it) that $\lim _{J} F_{\alpha}$ is a closed subscheme of $\prod_{J_{\max }} F_{\alpha}$. We also note that this scheme was previously considered in [KSZ, Sec.4].

TheOREM 2.11.8. There are natural morphisms $M \rightarrow F_{\alpha}$ compatible with the morphisms $F_{\alpha_{1}} \rightarrow F_{\alpha_{2}}$ and, hence, a morphism $f: M \rightarrow M_{\text {simp }}$.

Proof. Fix $q \in Q$. For any family $(P, L, \theta) / S$ of stable toric pairs we will construct a map $S \rightarrow F_{q}$ in a functorial way. That will induce the morphism $M \rightarrow F_{q}$ from the moduli space. For any point $s \in S$ we will construct an affine neighborhood $\operatorname{Spec} R \ni s$ and a map $\operatorname{Spec} R \rightarrow F_{q}=\operatorname{Proj} \mathbb{Z}\left[H_{q}\right]=$ $\operatorname{Proj} \mathbb{Z}\left[H_{q}\right]^{(d)}$, where $\mathbb{Z}\left[H_{q}\right]^{(d)}$ is the subring of the graded ring $\mathbb{Z}\left[H_{q}\right]$ of elements of degrees divisible by $d N q$, for some $d>0$. This will be induced by a ring homomorphism $\mathbb{Z}\left[H_{q}\right]^{(d)} \rightarrow R$. Write $\theta \in H^{0}(P, L)$ as the sum of its homogeneous components: $\theta=\sum_{x \in C_{\max }} \xi_{x}$. For $f=\left(f_{x}\right), g=\left(g_{x}\right)$ with $\phi f=\phi g$ the sections $\xi^{f}=\prod \xi_{x}^{f_{x}}$ and $\xi^{g}$ both lie in the same eigenspace of $H^{0}\left(P, L^{d}\right), d=\operatorname{deg} f$. Let $s \in S$ be a point and $\kappa(s)$ be its residue field. Then on the fiber $P_{s}$ one of the residues of $\xi^{h}, h \in H_{q}, \operatorname{deg} h>0$, must be nonzero: $q$ lies in a cell $\delta$ of the decomposition $\Delta$ induced by the fiber $\left(P_{s}, L_{s}, \theta_{s}\right)$, and for this cell for any $\chi \in$ Cone $\delta$ there is an $h$ with $\phi h=k \chi$ and $\xi^{h} \neq 0$ : this is another way of saying that the group $\mathbb{K}_{\delta}$ is finite. Let $d$ be minimal such that there is a $\xi^{h}$ with $\phi h=d N q$ and nonzero residue. Choose a small affine neighborhood Spec $R^{\prime} \ni s$ and a generator $e$ in $H^{0}\left(\operatorname{Spec} R^{\prime}, L\right)_{d N q}=R^{\prime}$ so that we can write $\xi^{f}=c(f) e^{k}$. Further, for one fixed $h$ let $\operatorname{Spec} R$ be a smaller neighborhood so that for any $s^{\prime} \in \operatorname{Spec} R$ the residue of $c(h)$ in $\kappa\left(s^{\prime}\right)$ is not zero. Then the rule $f \mapsto c(f)$ defines the required homomorphism $\mathbb{Z}\left[H_{q}\right] \rightarrow R$ inducing the morphism $\operatorname{Spec} R \rightarrow F_{q}$. This morphism clearly does not depend 
on a choice of generator $e$, hence is the same on intersections, hence defines a unique morphism $S \rightarrow F_{q}$, as required. Compatibility with maps $F_{\alpha_{1}} \rightarrow F_{\alpha_{2}}$ is easily shown.

Now, let us fix a field $k$ and describe the $k$-points of $M_{\text {simp }}(k)$.

Lemma 2.11.9. $M_{\text {simp }}(k)$ is the disjoint union of $H^{0}(\Delta, \widehat{\mathbb{\mathbb { L }}})$ going over all subdivisions of $\left(Q, C_{\max }\right)$.

Proof. By the definition of $k$-points and inverse limit, $M_{\text {simp }}(k)=\lim F_{\alpha}(k)$. A point $p$ of the toric variety $F_{q}$ lies in one of the toric orbits which, in turn corresponds to a face of the polytope $\sigma_{q}$. It is easy to see that faces of $\sigma_{q}$ are in a 1-to- 1 correspondence with cells $\delta \subset Q$ with $q$ in the interior, and that the corresponding toric orbit is $\widehat{\mathbb{L}}_{\delta}$. If $\delta_{2}$ is a face of $\delta_{1}$ and $q_{2} \in \delta_{2}^{0}$ is a point then the image of $p$ under $F_{q_{1}}(k) \rightarrow F_{q_{2}}(k)$ is given by the homomorphism $\widehat{\mathbb{L}}_{1} \rightarrow \widehat{\mathbb{L}}_{2}$. Hence, we see that a point of $\lim F_{\alpha}(k)$ is given precisely by fixing a cell decomposition $\Delta$ and an element of $\underbrace{\lim } \widehat{\mathbb{L}}_{i}=H^{0}(\Delta, \widehat{\mathbb{\mathbb { L }}})$.

COROLlary 2.11.10. $\quad M$ and $M_{\text {simp }}$ have natural stratifications with strata in a 1-to-1 correspondence with decompositions $\Delta$ of $Q$. For every $\Delta$ these strata are isogenous.

Proof. By Lemma 2.2.7 the map $H^{1}\left(\widehat{\mathbb{M}}^{*}\right) \rightarrow H^{0}(\underline{\mathbb{\mathbb { L }}})$ has finite kernel and cokernel.

COROLlary 2.11.11. The morphism $M \rightarrow M_{\text {simp }}$ is finite. $M$ is projective over the base scheme.

Proof. Indeed, $M_{\text {simp }}$ is clearly projective: it is obtained from several projective schemes by taking fiber products. Hence, both schemes are proper and so the morphism is proper (our base scheme is separated). Since all geometric fibers are finite, it is finite, and so $M$ is also projective.

Definition 2.11.12. For every decomposition $(\Delta, C)$ of $(Q, Q \cap X)$ there is a natural open affine subscheme in $M_{\text {simp }}$ which is the spectrum of a semigroup algebra. Indeed, in each $F_{q}$ the cell $\delta \in(\Delta, C)$ containing $q$ in its interior defines a saturated sub-semigroup $K_{\delta, q}$ in $\mathbb{L}$ defined by the differences $\chi_{1}-\chi_{2}$ with $\chi_{2} \in$ Cone $\delta$. This gives an open affine subscheme Spec $\mathbb{Z}\left[K_{\delta, \alpha}\right]$ in $F_{\alpha}$. In the inverse limit we get an open affine subscheme

$$
U[\Delta, C]=\lim \operatorname{Spec} \mathbb{Z}\left[K_{\delta, \alpha}\right]=\operatorname{Spec} \mathbb{Z}\left[\underline{\lim } K_{\delta, \alpha}\right] .
$$

As $(\Delta, C)$ goes over all triangulations of $(Q, Q \cap X)$ we obtain an open cover of $M_{\text {simp }}$. 
2.12. Generalized secondary polytopes for finite complexes.

2.12.1. All polytopes $\sigma_{\alpha}$ lie in spaces which are parallel to $\mathbb{L}=$ $\operatorname{ker}\left(\operatorname{Fun}\left(C_{\max }, \mathbb{Z}\right) \rightarrow \mathbb{X}\right)$. Consider the Minkowski sum $\Sigma(Q)=\Sigma\left(Q, C_{\max }\right)=$ $\sum \sigma_{\alpha}$. The projective toric variety $F_{\Sigma(Q)}$ corresponding to this polytope and the lattice $\mathbb{L}$ maps to every $F_{\alpha}$ since every $\sigma_{\alpha}$ can be obtained from the Minkowski sum by degenerating and collapsing some faces. Therefore, it maps to the inverse limit, i.e. $M_{\text {simp }}$.

THEOREM 2.12.2. $\quad F_{\Sigma(Q)}$ maps isomorphically onto an irreducible component of $M_{\text {simp. }}$.

Proof. In semigroup terms, the morphism $f: f^{-1} U[\Delta, C] \rightarrow U[\Delta, C]$ corresponds to a semigroup homomorphism $\lim _{\longrightarrow} K_{\delta, q} \rightarrow \mathbb{L}$ and since every $K_{\delta, q}$ is saturated in $\mathbb{L}$, the image is also saturated. The semigroup image is what describes $\operatorname{im} f$. We note that $\operatorname{im} F_{\Sigma(Q)}$ maps to an irreducible component of $M_{\text {simp }}$ since both contain an open dense subset $\operatorname{Spec} \mathbb{Z}[\mathbb{L}]$.

Corollary 2.12.3. $\quad F_{\Sigma(Q)}$ maps isomorphically onto an irreducible component of $M$.

Proof. Let $M^{\prime}=M\left[\Delta_{\max }, C_{\max }\right]$ be the reduced part of the main irreducible component of $M$. Both $M^{\prime}$ and $F_{\Sigma(Q)}$ have finite morphisms to the

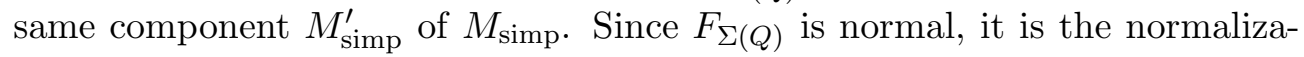
tion of $M^{\prime}$. On the other hand, the composition $F_{\Sigma(Q)} \stackrel{\psi}{\rightarrow} M^{\prime} \rightarrow M_{\text {simp }}^{\prime}$ is an isomorphism; hence $\psi$ is also an isomorphism.

The Minkowski average of the fibers of $\sigma \rightarrow Q$ is precisely the secondary polytope of Gelfand-Kapranov-Zelevinsky. There is a more "balanced" version of it which is combinatorially equivalent to it and has parallel faces (hence, corresponds to the same toric variety) given by the following explicit formula:

Definition 2.12.4. For each triangulation $\operatorname{Tr}=(\Delta, C)$ of $(Q, X \cap Q)$, i.e. a pointed decomposition for which all the cells are simplices and $C$ consists precisely of the vertices of these simplices, let $\phi_{\operatorname{Tr}}$ be an element of $\operatorname{Fun}(C, \mathbb{Z})$ such that

$$
\phi_{\operatorname{Tr}}(x)=\sum_{\delta_{i}: x \in \delta_{i}} \operatorname{Vol} \delta_{i}
$$

$\Sigma(Q)=\Sigma\left(\Delta_{\max }, C_{\max }\right)$ is the convex hull in the space $\operatorname{Fun}(C, \mathbb{R})$ of the points $\phi_{\operatorname{Tr}}$ as $\operatorname{Tr}$ goes over all triangulations. 
Definition 2.12.5. Alternatively, $\Sigma(Q)$ is the Minkowski integral, dilated by $(r+1)$, of the fibers of all fibers $\sigma_{q}, q \in Q$. Indeed, the coordinates of the vertices above can be obtained by averaging the coordinates of the vertices in Lemma 2.11.3. This is a particular case of the fiber polytope construction of Billera and Sturmfels, [BS].

Definition 2.12.6. Choose a system of heights which is an arbitrary realvalued function $\psi: X \cap Q \rightarrow \mathbb{R}$, i.e. an element of $\operatorname{Fun}\left(C_{\max }, \mathbb{R}\right)$. Consider the lower envelope of the convex hull of the rays $\{(x, h) \mid x \in X \cap Q, h \geq \psi x\}$ in $X_{\mathbb{R}} \oplus \mathbb{R}$. The projections of the faces of this lower envelope define a subdivision $\Delta$ of $Q$. The set $C$ is the subset of $Q \cap X$ of the points where the corresponding point $(x, \psi x)$ lies on the boundary of $\mathrm{ConvHull}_{\psi}$, i.e. on the lower envelope. A subdivision $(\Delta, C)$ of this type has been called, in [GKZ], regular or coherent.

These definitions have already appeared in our description of the 1-parameter degenerations of stable toric pairs in Section 2.8.

2.12.7. For a polytope $\sigma_{\alpha}$ denote by $\operatorname{Fan}_{\alpha}$ its dual fan in $\operatorname{Hom}\left(\mathbb{L}_{\mathbb{R}}, \mathbb{R}\right)$. Recall that its 1-dimensional cones are the interior normals of codimension faces of $\sigma_{\alpha}$ and several such rays form a cone if and only if the corresponding faces intersect. One of the basic facts about Minkowski sums is that the fan $\operatorname{Fan}_{\Sigma(Q)}$ is obtained by intersecting all $\operatorname{Fan}_{\alpha}$.

Also basic is how the faces of the Minkowski sum (and therefore the cones in the intersection of fans) are obtained. For every $\bar{h} \in \operatorname{Hom}\left(\mathbb{L}_{\mathbb{R}}, \mathbb{R}\right)$, i.e a restriction of a height function $h$, for each $\sigma_{\alpha}$ one picks a face $F_{\alpha, \bar{\psi}}$ on which $h$ takes the minimum. Then the face corresponding to $h$ in the Minkowski sum $\Sigma(Q)$ is the Minkowski sum $\sum F_{\alpha, \bar{\psi}}$.

This implies that faces of the secondary polytope correspond bijectively to the regular subdivisions of $Q$. In particular, the vertices bijectively correspond to the regular triangulations.

Below is our generalization of the above combinatorial constructions. These generalized polytopes describe other irreducible components in our moduli space and its simplification.

We now fix an arbitrary subdivision $(\Delta, C)=\left\{\left(Q_{i}, C_{i}\right)\right\}$ of $(Q, X \cap Q)$.

Definition 2.12.8. The generalized secondary polytope $\Sigma(\Delta, C)$ is the image in $C_{0}\left(\underline{\mathrm{Fun}}_{\mathbb{R}}\right) / B_{0}\left(\underline{\mathbb{L}}_{\mathbb{R}}\right)$ of the direct product of the secondary polytopes $\Sigma(Q)_{i}$ in $C_{0}\left(\underline{\mathrm{Fun}}_{\mathbb{R}}\right)$. Alternatively, consider the image of the direct product $\prod \sigma_{i}$ in $C_{0}\left(\underline{\operatorname{Fun}}_{\mathbb{R}}\right) / B_{0}\left(\underline{\mathbb{L}}_{\mathbb{R}}\right)$. This maps surjectively to the product of polytopes $Q_{i}$ in $C_{0}\left(\mathbb{X}_{\mathbb{R}}\right)$. The generalized secondary polytope is the (dilated by $(r+1)$ ) Minkowski integral of the fibers of this polytope map. 
Example 2.12.9. In the easiest situation, assume that all the codimension one faces of all $\left(Q_{i}, C_{i}\right)$ are simplices. Then $C_{1}\left(\underline{\mathbb{L}}_{\mathbb{R}}\right)=0$. Consequently, $B_{0}\left(\underline{\mathbb{L}}_{\mathbb{R}}\right)=0$ and $\Sigma(\Delta, C)$ is simply the direct product of the secondary polytopes $\Sigma(Q)_{i}$.

Definition 2.12.10. A regular sub-decomposition of $(\Delta, C)$ is defined by the system of heights $\psi_{i}$ in $C_{0}\left(\underline{\mathrm{Fun}}_{\mathbb{R}}\right) / B_{0}\left(\underline{\mathbb{L}}_{\mathbb{R}}\right)$. On each $Q_{i}$ it is defined as before, and it is easy to see that these fit on the intersections. In other words, we consider the system of heights $\psi_{i}$ which on the intersections of $Q_{i}$ 's may differ, but only by linear functions (nonhomogeneous on $X_{\mathbb{R}}$, which is the same as homogeneous linear on $\mathbb{X}_{\mathbb{R}}$ ).

We note that by the result of Section 2.8 these are precisely the subdivisions of $(\Delta, C)$ that appear for the 1-parameter degenerations of a pair of type $(\Delta, C)$. The same argument as above about the faces of the Minkowski sum gives the following:

Lemma 2.12.11. There is a bijective correspondence between the faces of the generalized secondary polytope $\Sigma(\Delta, C)$ and regular subdivisions of $(\Delta, C)$.

Remark 2.12.12. Every regular decomposition of $(Q, X \cap Q)$ which is a sub-decomposition of $(\Delta, C)$ is a regular decomposition of $(\Delta, C)$, because we can take the same heights. The opposite is not true. Every regular decomposition of $(\Delta, C)$ defines a collection of regular decompositions of $\left(Q_{i}, C_{i}\right)$. The opposite is not true as well.

2.12.13. Give the vector space $C_{0}\left(\Delta, \underline{\operatorname{Fun}}_{\mathbb{R}}\right) / B_{0}\left(\Delta, \underline{\mathbb{L}}_{\mathbb{R}}\right)$ the integral structure by the lattice $\left(C_{0}(\Delta, \underline{\text { Fun }}) / B_{0}(\Delta, \underline{\mathbb{L}})\right) /$ torsion. We can consider the toric scheme $F_{\Sigma(\Delta, C)}$ corresponding to this polytope and the above lattice. Again, there is a natural morphism $F_{\Sigma(\Delta, C)} \rightarrow M_{\text {simp }}$. This time, however, we can only claim that it is finite to image because for a face $F$ the lattice $X_{F}$ may contain the corresponding lattice $\mathbb{L}_{i}$ for one of the polytopes $\sigma_{\alpha}$ as a sublattice of finite index. Therefore, $F_{\Sigma(\Delta, C)}$ and an appropriate closed subset of $M$ differ "in finitely many ways".

2.13. The moment map for the moduli space. Consider now the case of the base field $\mathbb{C}$. For any semigroup $H$ let $X=\operatorname{Spec} \mathbb{C}[H]$. Then the moment map Mom $: X(\mathbb{C}) \rightarrow \operatorname{Mom} X$ is defined to be the quotient map by the equivalence relation: two points $p_{i}: \mathbb{C}[H] \rightarrow \mathbb{C}$ coincide if $\left|p_{1}(h)\right|=\left|p_{2}(h)\right|$ for all $h \in H$. If a variety $Y$ is glued from several varieties $\operatorname{Spec} \mathbb{C}\left[H_{i}\right]$ 's and the gluing maps are given by monomials then the equivalence relation is preserved and the moment map is globally defined. This is the case, for example, for toric varieties, and it is well-known that the image of a projective toric variety can be naturally identified with a lattice polytope. 
2.13.1. Both $M=\operatorname{TP}^{\mathrm{fr}}[Q]$ and $M_{\text {simp }}$ were defined in semigroup terms. They are covered by affine schemes corresponding to semigroup algebras, and the gluing maps are given by monomials. Therefore, we have moment maps defined. Moreover, since all strata differed only by a finite group of multiplicative type, i.e. $\mu_{n_{1}} \times \cdots \times \mu_{n_{k}}$, the images of the moment maps of $\operatorname{Mom} M(\mathbb{C})$ and $\operatorname{Mom} M_{\text {simp }}(\mathbb{C})$ are exactly the same.

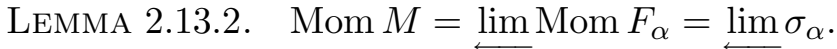

Proof. Indeed, it is clear that lim commutes with taking the quotient by the above equivalence relation.

TheOREM 2.13.3. Mom $M$ is obtained by gluing the generalized polytopes $\Sigma(\Delta, C)$ (some of these polytopes may appear as faces of others, others may be truly new).

Proof. This follows at once from the results of the previous subsection.

2.14. Relation to families of Gelfand-Kapranov-Zelevinsky.

2.14.1. Consider a pair $(P, \Theta)$ over a closed field $k$ that corresponds to a decomposition $(\Delta, C)$. As before, a section $\theta \in H^{0}(P, L)$ defining the divisor $\Theta$ can be written uniquely as the sum of its homogeneous components, i.e., eigenfunctions

$$
\theta=\sum_{x \in X \cap Q} \xi_{x}
$$

Therefore, we have $n=\#(Q \cap X)$ sections $\xi_{x}$ and the corresponding rational map $f_{\theta}: P-\rightarrow \mathbb{P}\left(H^{0}(P, L)^{*}\right)$.

Lemma 2.14.2. $\quad f_{\theta}$ is a finite morphism. The image is a cycle with multiplicities $\sum d_{i} V_{i}$, where $d_{i}$ is the order of the group $\mathbb{K}_{i}$.

Proof. Look at the restriction of $f_{\theta}$ to an irreducible component $P_{i}$. The condition that this restriction is a morphism is equivalent to the condition that $\xi_{x} \neq 0$ for all the vertices of the polytope $\delta_{i}$; this is precisely our condition on the divisor $\Theta$. The computation of the multiplicity is standard.

Fixing an origin in the main orbit of the $\mathbb{T}$-action on $L_{i}$ identifies $P_{i}$ with the standard projective variety $(P, L)[Q]$, and $H^{0}\left(P_{i}, L_{i}\right)$ - with the standard vector space $\operatorname{Fun}\left(\delta_{i} \cap X, k\right)=k^{n_{i}}$. Now choosing a different origin will change the isomorphism $H^{0}\left(P_{i}, L_{i}\right)^{*} \stackrel{\sim}{\rightarrow} k^{n_{i}}$. However, it will not change the image $f_{\theta}(P)$ since it is $T$ - and $\mathbb{T}$-invariant. Therefore, the cycle $\sum d_{i} V_{i}$ does not depend on the choice of origins made.

2.14.3. In their book [GKZ] and in the previous papers referenced there Gelfand-Kapranov-Zelevinsky consider a possibly nonnormal toric variety $V$ which is the image of the standard toric variety $P[Q]$ as described above, the 
cycles obtained from it by the action of the big torus $\left(k^{*}\right)^{n}$ and the cycles which are obtained as toric degenerations of these. They show that the extreme toric degenerations correspond to the Chow forms of weight $\phi_{\operatorname{Tr}}$ (with respect to the character group of the big torus, i.e. $\operatorname{Fun}(X \cap Q, \mathbb{Z})$ ), for all the regular decompositions Tr. Moreover, they show that the parameter space of the cycles that appear (as the result of the toric action and toric degenerations) is a possibly nonnormal toric variety corresponding to the polytope $\Sigma(Q)$.

2.15. Other families. Because of combinatorial restrictions on lattice polytopes $\delta$ - integral points in some generate the lattice and the semigroup of integral points in Cone $\delta$, and for some it is not true - it seems inevitable that we should either have

1. nice fibers in the families but a somewhat complicated moduli space, or

2. a nice parameter space but somewhat complicated fibers.

In this paper we have chosen the first solution, in part because we can compute the cohomologies $H^{p}\left(P, L^{d}\right)$ and it just seems to be more reasonable for the objects we are trying to parametrize to be as nice as possible. But, taking the second point of view one can construct at least two types of families, of schemes and not just the cycles, over the projective toric scheme $P_{\Sigma(Q)}$ corresponding to the secondary polytope.

2.15.1. (Family of the first type). Take a regular decomposition $(\Delta, C)$ of $(Q, Q \cap X)$. There is a corresponding face $F[\Delta, C]$ of $\Sigma(Q)$ and the corresponding fiber semigroup $K[\Delta, C]$ consisting of all differences $\chi_{1}-\chi_{2} \in \mathbb{L}$ with $\chi_{1} \in \operatorname{Cone} \Sigma(Q), \chi_{2} \in$ Cone $F[\Delta, C]$. Since $\Sigma(Q)$ is a Minkowski sum of fibers $\sigma_{q}$, for every $q \in Q$ there is a fiber group $K_{\delta, q}$ contained in $K[\Delta, C]$. Now consider the semigroup algebra over $\mathbb{Z}[K[\Delta, C]]=\oplus_{k \in K[\Delta, C]} \mathbb{Z} \zeta^{k}$ generated by variables $\xi_{x}, x \in Q \cap X$ with the following relations:

$$
\prod \xi_{x}^{m_{x}}=\prod \xi_{x}^{n_{x}} \times \zeta^{\left(m_{x}\right)-\left(n_{x}\right)}
$$

whenever $\left(m_{x}\right)-\left(n_{x}\right) \in K[\Delta, C]$. Define the family as the Proj of this algebra. Intersection of two families over $K\left[\Delta_{1}, C_{1}\right]$ and $K\left[\Delta_{1}, C_{1}\right]$ is identified with the one over $K\left[\operatorname{lub}\left(\left(\Delta_{1}, C_{1}\right),\left(\Delta_{2}, C_{2}\right)\right)\right]$ so they glue to a global family over the projective scheme.

2.15.2. (Family of the second type). This is defined to be the normalization of the first family.

Both of these familes are different from the ones we are considering. The generic fiber of the first need not be normal, and the flatness of the second is somewhat unclear. Both of these families may and do have some nonreduced fibers. We note that in [Nam1] Y. Namikawa has constructed families of first 
and second type over the toroidal compactification of $A_{g}^{(2 n)}$ for the 2nd Voronoi decomposition with the principal level structure, $n \geq 3$. These are analogs of the above two families.

2.16. First nontrivial example. A well-known (the first, really) example when there are nonregular decompositions is the following configuration: $Q$ is a triangle and $C$ is a 6 -point set as in Figure 1 . There are exactly 14 nonregular decompositions of $(Q, C)$. Two of them are triangulations as shown in Figure 1 (they appear e.g. on p. 219 of [GKZ]). We call them "Escherian staircases": one of them always goes up, and the other always goes down.
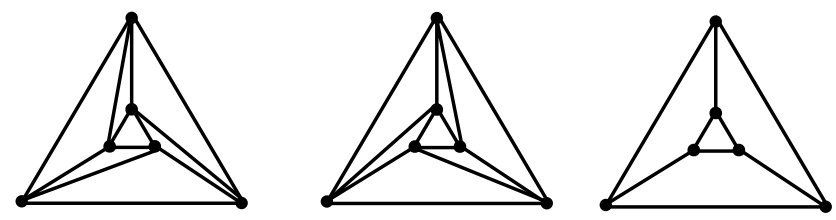

Figure 1. Some decompositions of the 6-point configuration.

The secondary polytope $\Sigma$ can be computed with some work, and it is combinatorially equivalent to the first polytope in Figure 2. For all regular decompositions $\Delta$ the secondary polytopes $\Sigma(\Delta)$ are the faces of $\Sigma(Q, Q \cap X)$ with one exception: when $\Delta$ is the third decomposition in Figure 1. The interior triangle $\delta_{0}$, being a simplex, has a point as its secondary polytope. The secondary polytopes for the quadrangulars $\delta_{1}, \delta_{2}, \delta_{3}$ are closed intervals. The space $H_{0}(\Delta, \mathbb{L})=\mathbb{L}_{1} \oplus \mathbb{L}_{2} \oplus \mathbb{L}_{3}$ is 3 -dimensional since $\mathbb{L}_{\delta}=0$ when $\delta$ is an interval, so that $C_{1}(\underline{\mathbb{L}})=0$. The secondary polytope $\Sigma(\Delta)$ in $L_{\Delta}$ is the direct product of three intervals, i.e. a cube, somewhat unrealistically shown in Figure 2. The projection of this cube to $\mathbb{L}_{C, \mathbb{R}}$, however, is 2-dimensional, and it is the hexagon lying in the foundation of $\Sigma$. Every cell decomposition of $(Q, C)$ corresponds to a face of one or both of these secondary polytopes.

The moduli interpretation of all this is as follows. The space $M_{(Q, A) \text { red }}$ has two irreducible components. Both are projective toric varieties for the polytopes in Figure 2, the second being simply $\mathbb{P}^{1} \times \mathbb{P}^{1} \times \mathbb{P}^{1}$. The first irreducible component parametrizes "smooth" (in fact only normal) and "smoothable" stable toric pairs $(P, \Theta)$.

A point $(a, b, c) \in \mathbb{P}^{1} \times \mathbb{P}^{1} \times \mathbb{P}^{1}$ corresponds to a smoothable pair if and only if $a b c=1$ (or $x_{0} y_{0} z_{0}=x_{1} y_{1} z_{1}$ in projective coordinates). The nonregular decompositions are the ones that violate this formula. For example, for the two triangulations in Figure 1 one has $0 \cdot 0 \cdot 0 \neq 1$ and $\infty \cdot \infty \cdot \infty \neq 1$ (on the other hand, $0 \cdot 0 \cdot \infty=1$, so to say, and thus gives a regular triangulation). 

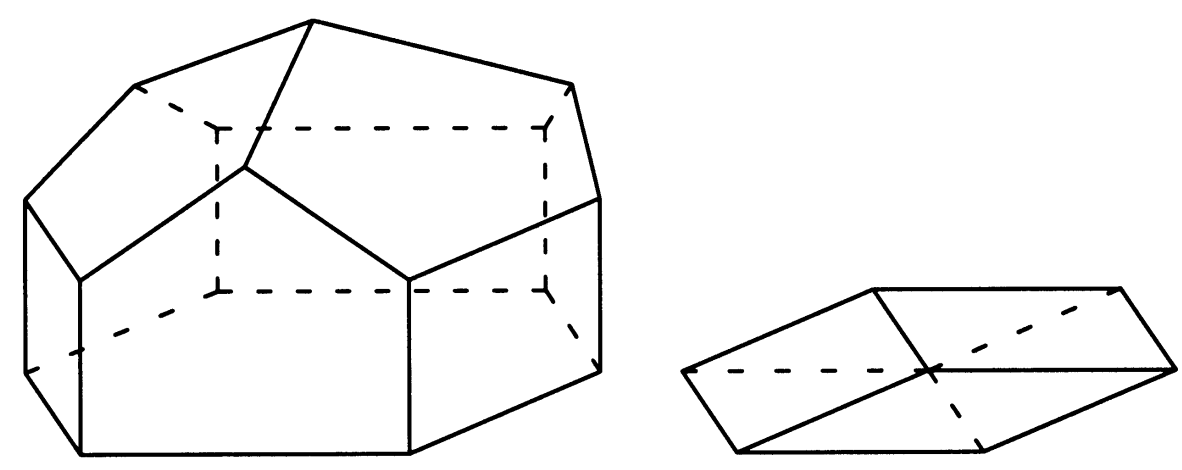

Figure 2. Two secondary polytopes of the 6-point example.

Note also that the intersection of two components is a blowup of $\mathbb{P}^{2}$ at three points.

The image of the moment map Mom $M$ in this case is the intersection of the above two polytopes, with the hexagonal foundation of the first polytope sitting in a "curved way" in the second.

2.16.1. Prof. W. J. Whiteley had pointed out to me that if we rotate the interior triangle slightly in Figure 1 then the third decomposition will no longer be regular. The secondary polytope in this case is the deformation of the one above obtained by adding a point under the foundation and connecting it to three of the six vertices above it. The second polytope is still a cube. However, the intersection of the polytopes in this case will consist of three square faces lying under the old foundation. We can adopt the above arithmetics to this case, too: a point $(a, b, c)$ corresponds to a smoothable pair if and only if $a b c=\infty$.

\section{Abelic pairs}

Lemma 3.0.1. Let $(A \curvearrowright P, L) / S$ be a polarized abelic scheme over $S$ of degree $d$. Then it induces a polarization $\lambda: A \rightarrow A^{t}=\mathbf{P i c}_{A / S}^{0}$ of degree $d$ on the abelian scheme $A$.

Proof. The invertible sheaf $L$ and the action $A \curvearrowright P$ define a polarization homomorphism $\lambda(L): A \rightarrow \mathbf{P i c}_{P / S}^{0}$; cf. 4.1.15. After an fppf (even étale) base change $S^{\prime} \rightarrow S$ we have an isomorphism $A^{\prime} \simeq P^{\prime}$ so that it is is obvious that $\operatorname{deg} \lambda=d^{2}$. The result now follows from the next theorem. 
Definition 3.0.2. Let $A$ be an abelian scheme over $S$ and let $B$ and $C$ be two schemes over $S$ with an $A$-action. $B \stackrel{A}{\times} C$ will denote the contracted product, i.e. the quotient $(B \underset{S}{\times} C) / A$ in the fppf topology with the normal action of $A$ on $B$ and the opposite to normal action on $C$. In particular, we can take $C=P$ and $B=\mathbf{P i c}_{A / S}$ on which $A$ naturally acts by translations: $(a, L) \mapsto T_{a}^{*} L$.

Theorem 3.0.3. Let $A$ be an abelian scheme over $S$ and $P$ be an A-torsor. Then

(i) There exists a canonical isomorphism

$$
\underline{\operatorname{Pic}}_{A / S} \stackrel{A}{\times} P \simeq \underline{\operatorname{Pic}}_{P / S} ;
$$

(ii) The action of $A$ on $A^{t}=\underline{\operatorname{Pic}}_{A / S}^{0}$ is trivial and it induces canonical isomorphisms

$$
A^{t} \simeq \underline{\mathrm{Pic}}_{P / S}^{0} \text { and } \underline{\mathrm{NS}}_{A / S} \simeq \underline{\mathrm{NS}}_{P / S}
$$

Definition 3.0.4. All abelic pairs over $S$ of dimension $g$ and degree $d$ form a category in which we let the arrows be isomorphisms only. In this way, we get a groupoid $\mathcal{A P}_{g, d}$ over the category of schemes. The automorphism classes of abelic pairs over $S$ form a set. This gives a functor $\mathrm{AP}_{g, d}$ : Schemes $\rightarrow$ Sets.

By Lemma 3.0.1 there exists a natural "forgetful morphism" from $\mathrm{AP}_{g, d}$ to the functor $\mathrm{A}_{g, d}$ of polarized abelian schemes of dimension $g$ and degree $d$, and the same for the groupoids.

Construction 3.0.5. Consider now the following object. The polarization $\lambda$ defines a distinguished component $\mathbf{P} \mathbf{i c}_{A / S}^{\lambda}$ of the scheme $\mathbf{P i c} \mathbf{i}_{A / S}$ whose geometric points correspond to line bundles on $A_{\bar{s}}$ of numerical type $\lambda$. This component is an $A^{t}$-torsor and is a smooth scheme over $S$. Since $A / S$ has a section, there is a universal Poincaré bundle $\mathcal{F}_{\lambda}$ on $\underset{S}{A} \times \mathbf{P i c}_{A / S}^{\lambda}$ with a rigidification $\left.\mathcal{F}_{\lambda}\right|_{0 \times \mathbf{P i c}_{A / S}^{\lambda}} \simeq \mathcal{O}_{\mathbf{P i c}_{A / S}^{\lambda}}$. We have a $\mathbb{P}^{d-1}$-bundle $\mathbb{P}\left(p_{2 *} \mathcal{F}_{\lambda}\right)$ over $\mathbf{P i c}_{A / S}^{\lambda}$ which classifies Cartier divisors on $A$ of numerical type $\lambda$. There is a relative Cartier divisor $\Theta_{\lambda}$ on it whose geometric points are divisors $D_{\bar{s}}$ on $A_{\bar{s}}$ with $0 \in D_{\bar{s}}$. Indeed, this condition is locally given by one equation, evaluation at the zero section.

Lemma 3.0.6 (Raynaud [Ray1], XIII.1.1). For every abelic pair $(A, P, \Theta) / S$ there is a natural A-equivariant morphism $\phi: P \rightarrow \mathbb{P}\left(p_{2 *} \mathcal{F}_{\lambda}\right)$ such that $\phi^{*}\left(\Theta_{\lambda}\right)=$ $\Theta$. When $S=\operatorname{Spec} k, k=\bar{k}$, for every $A / k$ there is a 1-to-1 correspondence between abelic pairs $(P, \Theta)$ over $k$ and the $A$-orbits of $\mathbb{P}\left(p_{2 *} \mathcal{F}_{\lambda}\right)$. 
Proof. Let $p^{\prime}$ be a functorial point of $P$. By this we mean, as usual, an $S^{\prime}$ point of $P$ for a scheme $S^{\prime} / S$, i.e., a section of $P^{\prime} / S^{\prime}$. It defines an isomorphism $A^{\prime} \rightarrow P^{\prime}$ sending the zero section to $p^{\prime}$. Via this isomorphism we have a relative Cartier divisor $\Theta^{\prime}$ on $A^{\prime}$ which gives a morphism $P^{\prime} \rightarrow \mathbb{P}\left(p_{2 *} \mathcal{F}_{\lambda}\right) \underset{S}{\times} S^{\prime}$. It is clearly $A$-equivariant. This gives a morphism of functors and, by Yoneda's lemma, a morphism of schemes representing them. Also, the points mapping to $\Theta_{\lambda}$ are exactly the points contained in $\Theta$ and the preimage of the local equation of $\Theta_{\lambda}$ is the local equation of $\Theta$.

COROLlaRY 3.0.7. In the principally polarized case the groupoids $\mathcal{A P}_{g}$ of abelic pairs and $\mathcal{A}_{g}$ of abelian varieties are naturally equivalent.

Proof. Indeed, in this case $\mathbb{P}\left(p_{2 *} \mathcal{F}_{\lambda}\right)=\mathbf{P i c}_{A / S}^{\lambda}$ is an $A=A^{t}$-torsor, and the above morphism is an isomorphism.

Remark 3.0.8. Recall that $\mathcal{A}_{g}$ is a smooth Mumford-Deligne stack.

COROLlary 3.0.9. There exists a coarse moduli space $\mathrm{AP}_{g}$ of principally polarized abelic pairs and it is isomorphic to $\mathrm{A}_{\mathrm{g}}$.

3.0.10. Let us emphasize again how this equivalence of stacks works. For any family $(A \curvearrowright P \supset \Theta) / S$ we already have the abelian scheme $A / S$, and the principal polarization $\lambda: A \rightarrow A^{t}$ is given by Lemma 3.0.1. Vice versa, given a family $\left(A, \lambda: A \rightarrow A^{t}\right) / S$ we take $P=\mathbf{P i c}_{A / S}^{\lambda}$ and by the above construction it comes with a natural relative Cartier divisor.

Remark 3.0.11. A choice of a section $p: S \rightarrow P$ gives an isomorphism $\phi_{p}: A \rightarrow P$ sending the zero section of $A$ to $p$. Since in general a section $p$ need not exist, $A$ and $P$ need not be isomorphic. Even when a section does exist (for example, $S=\operatorname{Spec} k, k=\bar{k}$ ), there is no canonical isomorphism. On the other hand, note that by $[\mathrm{Mum} 2,6.2]$ for every $\lambda$ there is a canonical section of $\mathbf{P} \mathbf{i c}_{A / S}^{2 \lambda}$.

TheOREM 3.0.12. The stack $\mathcal{A P}_{g, d}$ is a separated Artin stack admitting a coarse moduli space which is a separated scheme. This is a Mumford-Deligne stack over $\mathbb{Z}[1 / d]$.

Proof. By the above lemma $\mathcal{A P}_{g, d}$ is the quotient stack $\left[\mathbb{P}\left(p_{2 *} \mathcal{F}_{\lambda}\right) / A\right]$ by the group action, where we consider both $\mathbb{P}\left(p_{2 *} \mathcal{F}_{\lambda}\right)$ and $A$ to be the stacks over $\mathcal{A}_{g, d}$. Going to an étale cover, we obtain $\mathcal{A P}_{g, d}$ as the quotient stack of a scheme by an appropriate equivalence relation. Since $A$ is proper over $\mathcal{A}_{g, d}$, this is a proper stack, and it also has finite stabilizer. Existence of the coarse moduli space as a separated algebraic space in this situation is established in $[\mathrm{KM}]$. Outside characteristics dividing $d$, the stabilizers are étale, and the quotient stack therefore is Mumford-Deligne. 
The association $(A, P, \Theta) / S \mapsto(A, \lambda) / S$ defines a morphism of stacks $\mathcal{A} \mathcal{P}_{g, d} \rightarrow \mathcal{A}_{\},\lceil}$and their coarse moduli spaces. Since $\mathbb{P}\left(p_{2 *} \mathcal{F}_{\lambda}\right)$ is a projective bundle of relative dimension $d-1$, the morphism $\mathrm{AP}_{g, d} \rightarrow \mathrm{A}_{g, d}$ is projective of relative dimension $d-1$. Since $\mathrm{A}_{g, d}$ is a scheme, so is $\mathrm{AP}_{g, d}$.

Over $\mathbb{Z}[1 / d]$ the moduli space $\mathrm{A}_{g, d}$, and hence also $\mathrm{AP}_{g, d}$, is the disjoint union of components corresponding to different types $\left(d_{1}, \ldots, d_{g}\right)$ with $\prod d_{i}=d$. Recall that this means that on every geometric fiber, $\operatorname{ker} \lambda: A \rightarrow A^{t}$ together with the associated symplectic form is isomorphic to $H \times \widehat{H}, H \simeq$ $\mathbb{Z}_{d_{1}} \times \cdots \times \mathbb{Z}_{d_{g}}$. We can formally associate with this component a complex $\Delta$ consisting of one cell $\bar{X}_{\mathbb{R}} / Y$ with $X / Y \simeq H$.

\section{Linearization of torus action}

Let $H$ be a connected algebraic group acting on a proper variety $P$ and let $L$ be an invertible sheaf on $P$. One of standard problems of geometric invariant theory is to find a linearization of $L$, i.e. to extend the $H$-action to $L$. A simple answer is that if $H$ does not have any characters, i.e. homomorphisms to $\mathbb{G}_{m}$, and if $P$ is normal then some power $L^{n}$ is uniquely linearizable, see [Mum2, Ch. $1, \S 3]$.

Here, we are concerned with the opposite situation: $H$ is a torus $T$, so that it is essentially built of characters, and $P$ is not necessarily normal (but is proper). The answer turns out to be quite interesting and nontrivial. First of all, when it is not empty, the set of linearizations is a torsor over the Cartier dual $X$ of $T$ (over a closed field, $X \simeq \mathbb{Z}^{r}$ ). Secondly, the linearization need not exist at all. However, we prove that if $P$ is seminormal then, in a canonical way, there is an infinite étale $T$-invariant cover $\widetilde{P} \rightarrow P$ on which the pullback $\widetilde{L}$ of $L$ is canonically linearizable. It comes with a properly discontinuous action of $X$ in Zariski topology, and one has $P=\widetilde{P} / X, L=\widetilde{L} / X$. If $L$ is linearizable (e.g. $P$ is normal) then $\widetilde{P}$ is not new: it is simply a disjoint union of many copies of $P$. However, if $L$ is not linearizable then $\widetilde{P}$ contains a connected component which is not of finite type!

To the author's knowledge, covers of this sort have made their first appearance in Mumford's construction [Mum1] where they are presented as ad hoc examples. Some schemes $\widetilde{P}$ are given first, and then schemes $P$ are obtained as quotients. We have arrived at the answers of this section largely by trying to understand the exact nature of examples in [Mum1]. This understanding will enable us to invert Mumford's construction and go from the scheme downstairs to its infinite cover.

The main result to establish is the equivalence of categories:

1. Families $(T \curvearrowright P, L)$ over some base $S$, with the action by a split torus $T / S=\widehat{X} / S$, with proper $P / S$ and relatively ample $L$, and with seminormal geometric fibers; 
2. Families $(T \curvearrowright \widetilde{P}, T \curvearrowright \widetilde{L})$ with a properly discontinuous in Zariski topology action $X \curvearrowright \widetilde{P}$ commuting with the $T$-action in a special way, such that $(P, L)=(\widetilde{P}, \widetilde{L}) / X$.

4.1. The polarization morphism and theorem of square.

A. The case of a closed field.

Setup 4.1.1. Consider

1. A proper variety $P$ defined over an algebraically closed field $k$. Recall that all our varieties are connected and reduced but not necessarily irreducible.

2. A connected smooth commutative group variety $G$ over $k$ acting on $P$ via $\sigma: G \times P \rightarrow P$.

3. An invertible sheaf $L$ on $P$.

Definition 4.1.2. We will say that theorem of square holds if for any $a, b \in G(k)$

$$
T_{a+b}^{*} L \otimes L \simeq T_{a}^{*} L \otimes T_{b}^{*} L
$$

Definition 4.1.3. The polarization morphism $\lambda(L): G \rightarrow \mathbf{P i c} P$ is defined by

$$
\lambda(L): a \mapsto T_{a}^{*} L \otimes L^{-1}
$$

Obviously, theorem of square holds if and only if the polarization morphism is a homomorphism.

Here Pic $P$ is a group scheme locally of finite type over $k$. The connected component of identity $\mathbf{P i c}{ }^{0} P$ is of finite type, as well as the group scheme $\mathbf{P i c}^{\tau} P$ classifying sheaves $M$ whose finite powers are algebraically equivalent to zero. $\left(\mathbf{P i c}^{0} P\right)_{\text {red }}$ is a group variety over $k$. In characteristic 0 one of course has $\mathbf{P i c}{ }^{0} P=\left(\mathbf{P i c}^{0} P\right)_{\text {red }}$. Since we assumed $G$ to be connected and reduced, $\lambda(L)$ factors through $\left(\mathbf{P i c}^{0} P\right)_{\text {red }}$.

The action $G \curvearrowright P$ induces the translation action $G \curvearrowright$ Pic $P$. If $a \in$ $G^{\prime}\left(S^{\prime}\right), G^{\prime}=G \times \underset{k}{ } S^{\prime}$ is a functorial point of $G$ for a $k$-scheme $S^{\prime}$ and $M$ is an invertible sheaf on $P^{\prime}=\underset{k}{P} S^{\prime}$ then

$$
T_{a}^{*} M=a^{*}\left(\sigma^{*} M\right) .
$$

Note that this action preserves the group law on $\mathbf{P i c} P$ and that $\mathbf{P i c}^{0} P, \mathbf{P i c}^{\tau} P$ and $\left(\mathbf{P i c}^{0} P\right)_{\text {red }}$ are all $G$-invariant.

4.1.4. Let us review the situations in which theorem of square is known: 
1. $G$ is an abelian variety: because any morphism from an abelian variety to a group variety taking zero to zero is a homomorphism. Moreover, this works in families, too: [Mum2, Cor. 6.4].

2. $\left(\mathbf{P i c}{ }^{0} P\right)_{\text {red }}$ is an abelian variety (which is the case if $P$ is nonsingular in codimension 1): because any morphism from a group variety to an abelian variety taking zero to zero is a homomorphism as well; see [Lan, II.1, Thm.4].

3. [Lan, III.3] also contains the following version of theorem of square: Let $D$ be a Weil divisor on an irreducible but not necessarily proper $P$. Then for any $a, b$ the cycle

$$
T_{a+b}^{*} D-T_{a}^{*} D-T_{b}^{*} D+D
$$

is rationally equivalent to zero.

This implies the version we want provided there are no rational maps from $\mathbb{P}^{1}$ to $\left(\mathbf{P i c}{ }^{0} P\right)_{\text {red }}$. This is the case, again, if $\left(\mathbf{P i c}{ }^{0} P\right)_{\text {red }}$ is an abelian variety.

We would like to have theorem of square in the next best case: when $G$ is semiabelian. Naively, one would hope that theorem of square always holds here, too.

Counterexample 4.1.5. Let $P$ be the cuspidal curve $y^{2} z=x^{3}$ with the parametrization $p_{t}=(x, y, z)=\left(t^{-2}, t^{-3}, 1\right), t \in \mathbb{P}^{1}$, together with the following torus action: for $a \in \mathbb{G}_{m}, T_{a}\left(p_{t}\right)=p_{a^{-1} t}$.

The Picard group Pic ${ }^{0} P$ is $\mathbb{G}_{a}$ and is identified with $P \backslash \operatorname{cusp}\{t=\infty\}$ via $t \mapsto\left[p_{t}-p_{0}\right]$. It is easy to see that $T_{a}^{*} \mathcal{O}\left(p_{t_{0}}\right)=\mathcal{O}\left(p_{a t_{0}}\right)$, so that $\lambda\left(\mathcal{O}\left(p_{t_{0}}\right)\right)(a)=$ $a t_{0}-t_{0} \in \mathbb{G}_{a}$. This is not a homomorphism unless $t_{0}=0$.

However, we will prove the following:

TheOrem 4.1.6. Let $G$ be a semiabelian variety and $P, L$ be as in 4.1.1. Assume that the unipotent rank of $\mathbf{P} \mathbf{i c}^{0} P$ is zero, i.e. it does not contain a copy of $\mathbb{G}_{a}$. Then theorem of square holds.

THEOREM 4.1.7. Let $P$ be a proper seminormal variety. Then the unipotent rank of $\mathbf{P i c}{ }^{0} P$ is zero; moreover, any morphism $\mathbb{A}^{1} \rightarrow \mathbf{P i c}^{0} P$ is constant.

LEMMA 4.1.8. Let $R$ be a reduced ring (commutative with 1 , of course) and $T$ be a free indeterminate. Then the subring of invertible elements $(R[T])^{*}$ coincides with $R^{*}$.

Proof. This is obvious for an integral ring, and hence for direct sums of integral rings. Since any reduced ring can be embedded into a direct sum of integral rings, we are done. 
Corollary 4.1.9. For any reduced scheme $X$ over $k$ one has $p_{1 *} \mathcal{O}_{X \times \mathbb{A}^{1}}^{*}$ $=\mathcal{O}_{X}^{*}$.

LEMma 4.1.10. Let $X$ be a reduced scheme over $k$ and $F$ be an invertible sheaf on $X \times \mathbb{A}^{1}$. Assume that there exists an open cover $\left\{U_{i}\right\}$ such that the restriction of $F$ to each $U_{i} \times \mathbb{A}^{1}$ is the pullback of an invertible sheaf on $U_{i}$. Then $F$ is the pullback of an invertible sheaf on $X$.

Proof. By the Leray spectral sequence we have the following sequence of cohomologies computed in Zariski topology:

$$
0 \rightarrow H^{1}\left(p_{1 *} \mathcal{O}_{X \times \mathbb{A}^{1}}^{*}\right) \rightarrow H^{1}\left(\mathcal{O}_{X \times \mathbb{A}^{1}}^{*}\right) \stackrel{\phi}{\rightarrow} H^{0}\left(R^{1} p_{1 *} \mathcal{O}_{X \times \mathbb{A}^{1}}^{*}\right) .
$$

The condition of the lemma says exactly that the image of the class $[F] \in$ $\operatorname{Pic}\left(X \times \mathbb{A}^{1}\right)$ under $\phi$ is zero. On the other hand, by the previous corollary the kernel is $H^{1}\left(p_{1 *} \mathcal{O}_{X \times \mathbb{A}^{1}}^{*}\right)=H^{1}\left(\mathcal{O}_{X}^{*}\right)=\operatorname{Pic} X$.

Proof of Theorem 4.1.7. A morphism $\mathbb{A}^{1} \rightarrow \mathbf{P i c} P$ induces a sheaf $F$ on $P \times \mathbb{A}^{1}$, the pullback of the universal bundle on $P \times \mathbf{P i c} P$. The morphism is constant if and only if $F$ is a pullback from $P$. By the previous lemma we only need to know this locally. The local statement is proved by Traverso, [Tra, $3.6]$.

Lemma 4.1.11. If the translation action of $G$ on $\left(\mathbf{P i c}^{0} P\right)_{\mathrm{red}}$ is trivial then theorem of square holds.

Proof. Indeed,

$$
\begin{aligned}
T_{a+b}^{*} L \otimes\left(T_{a}^{*} L\right)^{-1} \otimes\left(T_{b}^{*} L\right)^{-1} \otimes L & =T_{a}^{*}\left(T_{b}^{*} L\right) \otimes\left(T_{a}^{*} L\right)^{-1} \otimes\left(T_{b}^{*} L \otimes L^{-1}\right)^{-1} \\
& =T_{a}^{*}\left(T_{b}^{*} L \otimes L^{-1}\right) \otimes\left(T_{b}^{*} L \otimes L^{-1}\right)^{-1} .
\end{aligned}
$$

Since we assumed in 4.1.1 that $G$ is connected, $T_{b}^{*} L \otimes L^{-1}$ gives a point of $\left(\mathbf{P i c}^{0} P\right)_{\text {red }}$.

Lemma 4.1.12. Assume that $G$ is divisible, i.e. for every integer $n$ the homomorphism $n: G \rightarrow G$ of multiplication by $n$ is surjective. If the unipotent rank of $\left(\mathbf{P i c}{ }^{0} P\right)_{\text {red }}$ is zero then the translation action of $G$ on $\left(\mathbf{P i c}{ }^{0} P\right)_{\text {red }}$ is trivial.

Proof. It is well-known that $H=\left(\mathbf{P i c}^{0} P\right)_{\text {red }}$ in this case is a semiabelian variety itself. Indeed, every commutative group variety over $k=\bar{k}$ is an extension of an abelian variety by an affine group, which in turn is obtained by extending a torus by a unipotent group, which in turn has a filtration with every quotient isomorphic to $\mathbb{G}_{a}$.

For an integer $m$ denote ${ }_{m} H=\operatorname{ker}(m: H \rightarrow H)$. Since the $G$-action preserves the group law, each ${ }_{m} H$ is $G$-invariant. The action $G \curvearrowright{ }_{m} H$ is trivial. Indeed, it is equivalent to a homomorphism $G \rightarrow \operatorname{Aut}\left({ }_{m} H\right)$ and the 
latter is a finite group scheme over $k$. If we take $m$ to be coprime to chark then ${ }_{m} H$ and so also $\operatorname{Aut}\left({ }_{m} H\right)$ are reduced. Every point of it is annihilated by some $n$. On the other hand $n: G \rightarrow G$ is surjective. Hence $G \rightarrow \operatorname{Aut}\left({ }_{m} H\right)$ must be trivial. This argument works as well for any $m$, with geometric points replaced by functorial points.

Since the family $\left\{{ }_{m} H\right\}$ is dense in $H$ (where we can only take $m$ that are powers of an arbitrary integer coprime to chark) the action $G \curvearrowright H$ is trivial.

Proof of 4.1.6. Since a semiabelian group variety is divisible, we are done by $4.1 .12,4.1 .11$.

For the future, we will need the following:

LEMmA 4.1.13. Assume that $G$ is divisible. Then the action $G \curvearrowright$ $\left(\mathbf{P i c}{ }^{0} P\right)_{\mathrm{red}}$ is trivial if and only if the action $G \curvearrowright \mathbf{P i c}{ }^{\tau} P$ is trivial.

Proof. Indeed, $\mathbf{P i c}{ }^{\tau} P /\left(\mathbf{P i c}^{0} P\right)_{\text {red }}$ is finite. By the same argument as in the previous lemma the action of the divisible group on it must be trivial.

B. The case of families. Our next step is prove the result for families corresponding to 4.1.6. For this, we have to deal with questions about the relative Picard functor and multiplicative tori over arbitrary, locally noetherian schemes. When discussing these topics, one usually works with one of the Grothendieck topologies that is more flexible than the Zariski topology. The standard choice is the fppf (faithfully flat of finite presentation) topology. Indeed, by [SGA3, X] every torus becomes split on an fppf cover, and the relative Picard functor is defined by sheafifying the ordinary Picard functor in the fppf topology.

Hence, for the rest of this section when we say locally, it means locally in the fppf topology, and the cohomologies are those with respect to fppf topology. Note that for the statements above fppf can be replaced by étale. For smooth, locally of finite type, group schemes the cohomologies computed in the fppf and étale topologies coincide as well: [DE, IV.11.7].

Setup 4.1.14. 1. $P \rightarrow S$ is a proper flat morphism with geometrically reduced, connected seminormal fibers.

2. $G$ is a smooth commutative group scheme over $S$ with connected fibers acting on $P$ via the morphism $\sigma: G \underset{S}{\times} P \rightarrow P$.

3. $L$ is an invertible sheaf on $P$.

$\mathbf{P i c}_{P / S}$ will denote the relative Picard functor. If irreducible components of fibers of $P \rightarrow S$ are geometrically irreducible then by Mumford's theorem $\mathbf{P i c}_{P / S}$ is represented by a not necessarily separated scheme locally of finite 
presentation over $S$. On the other hand, if we instead assume that $P$ and $S$ are of finite type over a field or an excellent Dedekind domain then, according to M. Artin [Art1, 7.3], the relative Picard functor is represented by an algebraic space, locally of finite presentation over $S$. We refer to [BLR, Ch. 8] or [FGA] for more details about this.

The group scheme $G$ acts on $\mathbf{P i c} \mathbf{c}_{P / S}$ by translations: if $S^{\prime}$ is an $S$-scheme, and $a$ and $M$ are local sections of $G^{\prime}=G \underset{S}{\times} S^{\prime}$ and $\mathbf{P i c}_{P^{\prime} / S^{\prime}}=\mathbf{P i c}_{P / S} \underset{S}{\times S^{\prime}}$ respectively, then

$$
a \cdot M=T_{a}^{*} M=a^{*}\left(\sigma^{*} M\right) .
$$

This action preserves the group structure.

Definition 4.1.15. Let $L$ be an invertible sheaf on $P$. The polarization morphism $\lambda(L): G \rightarrow \mathbf{P i c}_{P / S}$ is defined by

$$
\lambda(L): a \mapsto T_{a}^{*} L \otimes L^{-1}
$$

for a local section $a$ of $G^{\prime}$.

Assume that we are in the one of the two basic situations above, so that $\mathbf{P i c}_{P / S}$ is a scheme (resp. algebraic space) locally of finite type over $S$. If $G$ is of finite type over $S$ and has connected fibers then $\lambda(L)$ factors through $\mathbf{P i c}^{\tau} P / S$ which is of finite type over $S$, and the above-defined action preserves $\mathbf{P i c}^{\tau} P / S$. Recall that the latter is the open subscheme (resp. algebraic space) of $\mathbf{P i c} \mathbf{i c}_{P / S}$ whose geometric points correspond to sheaves $M_{\bar{s}}$ on $P_{\bar{s}}$ with $M_{\bar{s}}^{n} \in \mathrm{Pic}^{0} P_{\bar{s}}$ for some $n \in \mathbb{N}$.

Definition 4.1.16. We say that theorem of square holds for $L$ if $\lambda(L)$ is a homomorphism. Equivalently, locally on $S$ we must have

$$
T_{a+b}^{*} L \otimes L \simeq T_{a}^{*} L \otimes T_{b}^{*} L
$$

Remark 4.1.17. This condition is weaker than asking the above two sheaves to be isomorphic or differ by an invertible sheaf on $S$.

As remarked in 4.1.4, if $G$ is an abelian scheme over $S$ then this more general form of theorem of square holds as an application of the rigidity lemma; see [Mum2, Cor. 6.4].

THEOREM 4.1.18. Let $G$ be a semiabelian group scheme over $S$, and $P, L$ be as in 4.1.14. Then the theorem of square holds for any invertible sheaf $L$ on $P$.

Remark 4.1.19. We use the assumption that every geometric fiber $P_{\bar{s}}$ is seminormal only to conclude that the unipotent rank of $\mathbf{P i c}{ }^{0} P_{\bar{s}}$ is zero. 
Proof. By the same exact argument as in 4.1.11, substituting geometric points by functorial points, we see that it suffices to prove that the action $G \curvearrowright \mathbf{P i c}^{\tau} P / S$ is trivial. We already know this statement for an algebraically closed field $\bar{k}$ : 4.1.12, 4.1.13. Since the question is local, this implies the statement for a nonclosed field $k$, too, so we have it for every closed fiber.

The main point is to prove the statement for a local artinian base $S$. It then gives the general case by a standard argument: Artinian case implies the case of a complete local ring, and since the base change from a local noetherian ring to its completion is faithfully flat, this implies the statement over the local ring $\mathcal{O}_{S, s}$, which clearly suffices.

Another reduction step is that, since the question is local, we can change to an fppf cover on which all irreducible fibers of $P_{s}$ are geometrically irreducible. Hence, we can assume that $\mathbf{P i c}{ }^{\tau} P / S$ is a scheme.

Now, the artinian case follows from 4.1.20.

LEMmA 4.1.20. Let $S$ be the spectrum of a local artinian ring with a closed point $S_{0}$. Let $G$ be a semiabelian group scheme over $S$ and let $Q$ be a scheme of finite type over $S$ on which $G$ acts via the morphism $\underset{S}{S} Q \rightarrow Q$. If the action $G_{0} \curvearrowright Q_{0}$ is trivial then the action $G \curvearrowright Q$ is trivial.

Proof. $G$ is an extension of an abelian scheme $A / S$ by a torus $T / S$. For the torus action we quote [SGA3, IX.3.8] where it is proved for any group scheme of multiplicative type.

Therefore, we are reduced to the case of abelian action $A \curvearrowright Q$. Let $s$ be a section of $Q$ over $S$. By restricting the action to $s$ we have a morphism

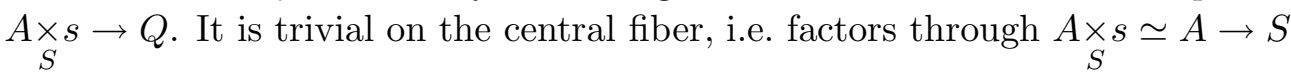
and $s: S \rightarrow Q$. By the rigidity lemma [Mum1, Prop.6.1] there is the same factorization globally. Since we also have this for any section after any local artinian base change $S^{\prime} / S$, it follows that the morphism $\underset{S}{\times} Q \rightarrow Q$ is the projection to the second component.

Next, we would like to analyze the kernel of the polarization morphism.

LEMma 4.1.21. Assume that $S$ is the spectrum of a DVR with the closed point 0 and the generic point $\eta$, and $G, P, L$ are as in 4.1.14. Let $H_{\eta}$ be a subgroup of $G_{\eta}$ and let $H$ be its closure in $G$. Denote by $\lambda_{H}$ the restriction of $\lambda$ to $H$. Then $\lambda_{H}\left(L_{\eta}\right)=0$ implies $\lambda_{H}(L)=0$.

Proof. Let $a \in H(S)$. The sheaf $M$ induces a morphism $\lambda(a): S \rightarrow$ $\mathbf{P i c}^{\tau} P / S$ and we want to show that it factors through the zero section. Combination of this with all base changes $S^{\prime} / S$ which can be assumed to be again DVRs, would prove the statement. 
The image of $\lambda(a)$ is contained in the closure $S_{1}$ of the zero section $S_{0}$. Note that $S_{1, \eta}=S_{0, \eta}$ and that $S_{1}$ is reduced. We claim that the fiber of $S_{1} \rightarrow S$ over 0 is a finite set. Indeed, for any affine open set $U \subset \mathbf{P i c}_{P / S}^{\tau}$ the closure of $S_{0, \eta}$ in $U$ has no more than one point over 0 since $U$ is separated, and $\mathbf{P} \mathbf{i c}_{P / S}^{\tau}$ is covered by finitely many such $U$ 's. Because $G_{0}$ is connected, $\lambda(a)(0)=0$ lies in $S_{0}$. Therefore, $\lambda(a)$ factors through $S_{0}$.

When we say a group scheme of multiplicative type we mean that locally in the fppf topology it is isomorphic to a product of several copies of $\mathbb{G}_{m}$ and $\mu_{n}$ and we do not necessarily assume that $n$ is coprime to the characteristics of the residue fields.

TheOREm 4.1.22. Let $G$ be a torus $T$ over $S$, and $P, L$ be as in 4.1.14. Then $\operatorname{ker} \lambda(L)$ is a flat and closed sub group scheme of $T$ of multiplicative type. The quotient $T / \operatorname{ker} \lambda(L)$ is a torus, and the polarization morphism factors through an immersion $T / \operatorname{ker} \lambda(L) \rightarrow \mathbf{P i c}^{\tau}{ }_{P / S}$.

Proof. Since the question is local, we can assume that $S$ is connected, Pic $_{P / S}^{\tau}$ is a scheme and that $T$ is diagonalizable: $T=\operatorname{Hom}_{S-\operatorname{gps}}\left(\mathbb{Z}_{S}^{r}, \mathbb{G}_{m, S}\right)$. For every $s \in S$ the subgroup ker $\lambda_{s} \subset T_{s}$ is closed and is also diagonalizable, i.e. of the form $\operatorname{Hom}\left(M_{s}, \mathbb{G}_{m, s}\right)$, where $M_{s}$ is a quotient of $\mathbb{Z}^{r}$. Denote by $H_{s}$ the corresponding diagonalizable subgroup of $T$.

Since we already know that $\lambda(L)$ is a homomorphism, by [SGA3, IX.5.2] there is an open subset $U \ni s$ of $S$ such that $H_{s} \subset \operatorname{ker} \lambda_{U}$. This implies that ker $\lambda$ is flat. Applying 4.1.21 to a base change by a DVR we see that it is also closed (if $\mathbf{P i c}{ }_{P / S}^{\tau}$ were separated that would be trivial). Hence, $\operatorname{ker} \lambda(L)=H$ for a diagonalizable subgroup of $T$.

The quotient $T / H$ is also diagonalizable, and so is a torus. The monomorphism $T / H \rightarrow \mathbf{P i c}^{\tau} P / S$ is an immersion by [SGA3, XVI.1.4].

Corollary 4.1.23. Under the conditions of the previous theorem, if $S$ is connected and for one geometric fiber $\lambda\left(L_{\bar{s}}\right)=0$ then $\lambda(L)=0$ globally.

4.2. Tori in Picard groups and infinite covers.

Example 4.2.1. Let $P$ be a projective rational curve with a single node defined over an algebraically closed field $k$. Let $L$ be an ample invertible sheaf on $P$, say of degree 1 . There is a standard action on $P$ of the multiplicative group $\mathbb{G}_{m, k}$ with two orbits: the node and its complement. It is easy to see that the polarization morphism $\lambda(L)$ in this case is a group isomorphism from $G$ to $\mathbf{P i c}^{0} P$.

Consider the scheme $\widetilde{P}$ which is the infinite chain of copies $C_{i}^{\nu} \simeq \mathbb{P}^{1}$, $i \in \mathbb{Z}$, with $C_{i}$ intersecting $C_{i-1}$ and $C_{i+1}$, as on the picture. The group $X=\mathbb{Z}$ naturally acts on $\widetilde{P}$ sending $C_{i}$ to $C_{i+x}$ and the singular points to singular points. It is easy to see that $P$ is the quotient $\widetilde{P} / X$. 


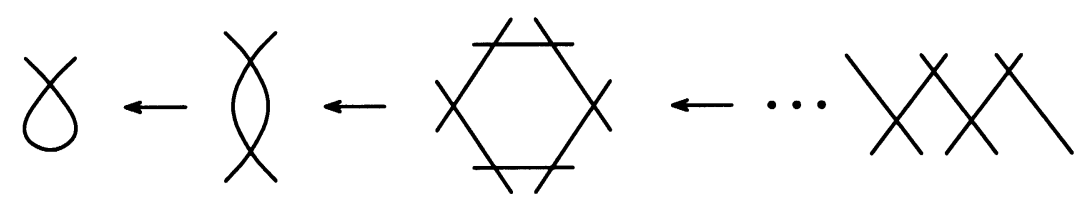

Quite interestingly, $\widetilde{P}$ is not of finite type. However, it is locally of finite type and the morphism $\widetilde{P} \rightarrow P$ is étale and Galois. Another series of examples is provided by Mumford's construction [Mum1]. We would like to understand the nature of such infinite covers.

The following statement and its corollary are well-known; see [Ray2, 6.2] (also [Mil, III.4.16]). Sheaves and cohomologies are in the fppf topology:

TheOREM 4.2.2. Let $f: P \rightarrow S$ be a proper morphism with geometrically reduced and connected fibers, and let $G$ be a finite flat commutative group scheme over $S$. Then there is a canonical isomorphism

$$
R^{1} f_{*} \widehat{G}_{P}=\underline{\operatorname{Hom}}_{S-\mathrm{gps}}\left(G, \mathbf{P i c}_{P / S}\right),
$$

where $\widehat{G}=\operatorname{Hom}_{S-\operatorname{gps}}\left(G, \mathbb{G}_{m, S}\right)$ is the Cartier dual of $G$, and $\widehat{G}_{P}=\widehat{G}_{S} P$.

Corollary 4.2.3. Let $P$ be a proper connected reduced scheme over an algebraically closed field $k$. Then there is a 1-to-1 correspondence between

(i) finite commutative subgroup schemes $G$ of $\mathbf{P i c} P$,

(ii) connected $\widehat{G}$-torsors $\widetilde{P}$ over $P$, i.e. connected schemes $\widetilde{P}$ over $S$ with a free $\widehat{G}$-action and with $\widetilde{P} / \widehat{G}=P$.

Proof. Fppf sheaves which are $\widehat{G}$-torsors are classified by $H^{1}(P, \widehat{G})$; cf. [Mil, III.4]. On the other hand, $\widehat{G}$ is affine, and affine sheaf torsors are always representable: [SGA1, VIII.7.9].

The example 4.2.1 is an application of 4.2.3, but with $G$ a multiplicative torus instead of being finite. Checking the proof of the above theorem, we see that it goes through for this case as well, with obvious changes.

TheOREM 4.2.4. Let $f: P \rightarrow S$ be a proper morphism with geometrically reduced and connected fibers, and let $T$ be a torus over $S$, a twist of $\mathbb{G}_{m, S}^{r}$, and let $X=\widehat{T}=\operatorname{Hom}_{S-\text { gps }}\left(T, \mathbb{G}_{m, S}\right)$ be its Cartier dual, a twist of the constant group $\mathbb{Z}_{S}^{r}$. Then there is a canonical isomorphism

$$
R^{1} f_{*} X_{P}=\underline{\operatorname{Hom}}_{S-\mathrm{gps}}\left(T, \mathbf{P i c}_{P / S}\right) .
$$


Proof. The same proof that is given in [Ray2,6.2] for the finite case (it is repeated in [Mil, 4.16] as well) works here verbatim. Aside from the Cartier duality, the only fact specific to the group $T$ is that $\underline{\operatorname{Ext}}^{1}\left(T, \mathbb{G}_{m, S}\right)=0$. This, in fact, is well-known. Let us recall the argument. We have to show that for any faithfully flat scheme $S^{\prime}$ over $S$ an extension $H$ of $\mathbb{G}_{m, S^{\prime}}$ by $T_{S^{\prime}}$ is locally split. But since both are of multiplicative type, by [SGA3, X] $H$ is also of multiplicative type, and therefore all three are locally diagonalizable. By taking the Cartier dual we obtain an exact sequence of constant groups, and that is obviously split on every connected component of the base scheme.

As for the corollary, we do not even have to assume that $k$ is closed:

COROLlaRY 4.2.5. Let $P$ be a proper connected reduced scheme over a field $k$ which has a $k$-rational point. Then there is a 1-to-1 correspondence between

(i) subgroups $T \subset \mathrm{Pic}_{P / k}^{0}$ which are tori,

(ii) connected $X$-torsors $\widetilde{P}$ over $P$, i.e. connected schemes $\widetilde{P}$ over $S$ with a free $\widehat{T}$-action and with $\widetilde{P} / \widehat{T}=P$.

Proof. An fppf sheaf which is an $X$-torsor over $P$ is again automatically representable. Indeed, this is proved in [SGA3, X.5.4] for a torsor with any twisted constant structure group. The sheaf torsors are classified by the first cohomology, as before. So, we have to prove the isomorphism $H^{1}(P, X) \simeq$ $\operatorname{Hom}\left(T, \mathbf{P i c}_{P / k}\right)$.

In the case of an algebraically closed field we are already done by 4.2.4. Over an arbitrary field $k$ we have the following exact sequence:

$$
H^{1}(k, X) \rightarrow H^{1}(P, X) \rightarrow H^{0}\left(k, R^{1} f_{*} X\right) \rightarrow H^{2}(k, X) \rightarrow H^{2}(P, X),
$$

and by $4.2 .4, H^{0}\left(k, R^{1} f_{*} X\right)=\operatorname{Hom}\left(T, \mathbf{P i c}_{P / k}\right)$. Hence, to prove the theorem it suffices to show that:

(i) $H^{1}(k, X)=0$.

(ii) The obstruction in $H^{2}(k, X) \rightarrow H^{2}(P, X)$ vanishes.

For the first assertion we have

$$
H^{1}(k, X)=\operatorname{Hom}(\operatorname{Gal}(\bar{k} / k), X)=0
$$

because $X$ is torsion-free and $\operatorname{Gal}(\bar{k} / k)$ is profinite.

As for the second statement, by a standard argument (cf. proofs of [Ray2, 6.2] or [Mil, 4.16]) obstructions of this type always vanish when there is a section, which in our case means a $k$-rational point. 
Remark 4.2.6. Note that neither object in 4.2 .5 exists when $P_{\bar{k}}$ is normal. Indeed, in this case every connected étale cover of $P$ is quasifinite (obviously since it is irreducible), and $\left(\mathrm{Pic}^{0} P\right)_{\text {red }}$ is an abelian variety (see [FGA, 6.2.1]).

Remark 4.2.7. Existence of infinite connected étale $\mathbb{Z}^{r}$-Galois covers of $P$ is closely related to existence of twists of the constant group $\mathbb{Z}^{r}$ over $P$ and the $r$-dimensional tori over $P$ which do not become trivial after any finite étale cover (by [SGA3, X.5.9] the latter two categories are anti-equivalent). Two examples in [SGA3, X.1.6] of a situation when this occurs are exact for the rational nodal curve as above and also for its étale double cover.

Example 4.2.8. To generalize 4.2.1, let $C=\cup_{i=1}^{N} C_{i}$ be a proper reduced connected curve with nodes only, for simplicity over an algebraically closed field $k$. It is well-known that $\operatorname{Pic}^{0} C$ is a semiabelian variety which fits into the following exact sequence

$$
0 \rightarrow T \rightarrow \mathrm{Pic}^{0} \mathrm{C} \rightarrow \mathrm{Pic}^{0} C^{\nu} \rightarrow 0,
$$

where $C^{\nu}$ is the normalization of $C$ and $T=\left(k^{*}\right)^{r}$. By assigning to each irreducible component $C_{i}$ a vertex and to each node an edge we obtain the dual graph $\Gamma$ of $P$. Then the dimension $r$ of the toric part is the cyclomatic number of $\Gamma$, the rank of $H_{1}(\Gamma)$ considered as a topological space, i.e., the number of "independent loops".

Consider the universal abelian cover $\widetilde{\Gamma}$ of $\Gamma$ corresponding to

$$
\pi_{1}^{a b}=\pi_{1} /\left[\pi_{1}, \pi_{1}\right]=\mathbb{Z}^{n}=\widehat{T}=X,
$$

where $\pi_{1}=\pi_{1}(\Gamma)$. This is an infinite graph with vertices marked $C_{1}^{\nu}$ through $C_{N}^{\nu}$. In an obvious way one constructs a scheme $\widetilde{C}$ corresponding to it which is a connected scheme locally of finite type with a morphism to $C$, so that $\widetilde{C} \rightarrow C$ is étale Galois with the group $X$.

4.3. Infinite covers arising from the torus action. Let $S$ be a locally noetherian scheme. The aim of this section is to prove the following:

THEOREM 4.3.1. The following categories are naturally equivalent:

(1) triples $(T, P, L) / S$, where

(a) $T$ is a split torus over $S$ with a Cartier dual $X_{S}=\mathbb{Z}_{S}^{r}$.

(b) $P$ is a proper scheme over $S$ with geometrically connected, reduced and seminormal fibers, with a T-action.

(c) $L$ is a relatively ample invertible sheaf on $P$.

(2) triples $(T, \widetilde{P}, \widetilde{L}) / S$, where

(a) $T$ is a split torus over $S$ with Cartier dual $X$. 
(b) $\widetilde{P}$ is a scheme, locally of finite type, over $S$ with geometrically reduced and seminormal fibers, with commuting actions of $T$ and $X$.

(c) $\widetilde{L}$ is a relatively ample invertible sheaf on $P$, with the actions of $T$ and $X$ extending those on $\widetilde{P}$.

such that

(a) $X$-action is properly discontinuous in the Zariski topology, and $P=$ $\widetilde{P} / X$ is proper over $S$ with geometrically connected fibers.

(b) The actions of $T$ and $X$ on $\widetilde{L}$ commute in the following way: if a and $x$ are functorial points of $T$ and $X$ (i.e. sections after a base change $\left.S^{\prime} / S\right)$, then

$$
T_{a}^{*} S_{x}^{*}=x(a) \cdot S_{x}^{*} T_{a}^{*}
$$

Definition 4.3.2. A sheaf $\widetilde{L}$ on a scheme $\widetilde{P}$ which is not necessarily of finite type over $S$ is called relatively ample if there exists an open cover $\left\{S_{i} \subset S\right\}$ such that for each $i$ the sections in $H^{0}\left(\widetilde{P}_{i}, \widetilde{L}_{i}^{d}\right), d \gg 0$, form a basis of topology on $\widetilde{P}_{i}$.

Example 4.3.3. Let $P$ be a projective $r$-dimensional toric variety over a closed field $k$. The polarization morphism $\lambda(L): T \rightarrow$ Pic $P$ is constant: $\lambda(L)=0$. An ample line bundle $L$ on $P$ can be linearized in infinitely many ways, each equivalent to giving a lattice polytope $Q$ in $X_{\mathbb{R}} \simeq \mathbb{R}^{r}$. The difference between any two such polytopes is a shift by a lattice vector $x \in X: Q_{2}=$ $Q_{1}+x$. Speaking invariantly, we have $\widetilde{P}$ as in the above theorem which is simply a disjoint union of $X$ copies of $P$ on which the pullback $\widetilde{L}$ is canonically linearized.

Of course, this example is rather trivial, and the situation is a lot more interesting when the cover $\widetilde{P}$ is not of finite type as in Example 4.2.1. However, we would like to show that these examples are both special cases of a general phenomenon provided by the above theorem. We would also like to understand the number of the connected components of $\widetilde{P}$.

Proof of Theorem 4.3.1. Given the pair $(\widetilde{P}, \widetilde{L})$ we define $P$ and $L$ as $\widetilde{P} / X$ and $\widetilde{L} / X$. Because the action is properly discontinuous in Zariski topology, for a subgroup $X^{\prime} \subset X$ of finite index there exists a system of neighborhoods $\left\{U_{i}\right\}$ of $\widetilde{P}$ such that for any $x^{\prime} \neq 0$ in $X^{\prime}$ one has $U_{i} \cap T_{x^{\prime}} U_{i}=\emptyset$. So, the quotient $\widetilde{P} / X^{\prime}$ is defined as a scheme, and $P$ is defined at least as an algebraic space. But it comes with an ample sheaf $L$, and so $P$ is actually a scheme. The opposite direction, going from $(P, L)$ to the cover $(\widetilde{P}, \widetilde{L})$ is more complicated. 
By 4.1.22 we have a subtorus $T_{Y}=\operatorname{im} \lambda(L)(T)$ in $\mathbf{P i c}^{\tau} P / S$. Let $Y$ be its Cartier dual. Dually, we have a closed subscheme $Y_{S} \subset X_{S}$. Theorem 4.2.4 provides that locally the subtorus $T_{Y}$ gives a $Y$-torsor $P^{\dagger}$ over $P$. In other words, there exists an fppf cover $\left\{S_{i} \rightarrow S\right\}$ such that the subtori $T_{Y, i}=T_{Y} \underset{S}{\times} S_{i} \subset \mathbf{P i c}^{\tau} P_{i} / S_{i}$ induce the $Y$-torsors $P_{i}^{\dagger}$ over $P_{i}=\underset{S}{P} \underset{S}{S_{i}}$. First, we are going to show that the action of $T$ lifts to each $P_{i}^{\dagger}$ and that each $L_{i}^{\dagger}$ is linearizable.

Let $G_{i}^{\dagger}$ be the fppf sheaf of isomorphisms of the $Y_{i}$-torsor $P_{i}^{\dagger}$ covering the $T_{i}$-action on $P_{i}$. We have a subgroup $Y_{i}=\underset{S}{\times} S_{i} \subset G_{i}^{\dagger}$ and we would like to show that $G_{i}^{\dagger} \rightarrow T_{i}$ is surjective. Let a be a local section of $T_{i}=T \underset{S}{S} S_{i}$. Because $\lambda(L)$ is a homomorphism, the translation action by a on $\mathbf{P i c}^{\tau}{ }_{P_{i}} / S_{i}$ induces the trivial action on $T_{Y, i}$. Indeed, locally for every $M=T_{b}^{*} L \otimes L^{-1}$ we have $T_{a}^{*} M \simeq M$. This implies that the torsors $P_{i}^{\dagger}$ and $T_{a}^{*} P_{i}^{\dagger}$ are isomorphic, so that the above homomorphism is surjective. Therefore, we have the exact sequence

$$
0 \rightarrow Y_{i} \rightarrow G_{i}^{\dagger} \rightarrow T_{i} \rightarrow 1
$$

On the other hand, $\operatorname{Ext}^{1}\left(T_{i}, Y_{i}\right)=0$ which follows because $\operatorname{Ext}^{1}\left(\mathbb{G}_{m}, \mathbb{Z}\right)=0$ (cf. the proof of [SGA71, 3.4]). Therefore, $G_{i}^{\dagger} / S_{i}$ is smooth, and locally there is a splitting defined by mapping $T_{i}$ to the connected component of the identity $\left(G_{i}^{\dagger}\right)^{0}$. Clearly, it glues to a global splitting. Hence, $G_{i}^{\dagger}=Y_{i} \times T_{i}$ and $\left\{P_{i}^{\dagger}\right\}$ is a $T$-invariant cover of $P$.

Consider a finite flat subgroup $H_{i}$ of $T_{Y, i}$. Dually, there is a subgroup $Y_{i}^{\prime}$ of $Y_{i}$ of finite index. The scheme $P_{i}^{\prime}=P_{i}^{\dagger} / Y_{i}^{\prime}$ is proper over $S_{i}$ and it is an $\widehat{H}_{i}$-torsor over $P_{i}, \widehat{H}_{i}=Y_{i} / Y_{i}^{\prime}$. Denote by $L_{i}^{\prime}$ the pullback of $L_{i}$ to $P_{i}^{\prime}$. The polarization morphism $\lambda\left(L_{i}^{\prime}\right): T_{i} \rightarrow \mathbf{P i c}^{\tau} P_{i}^{\prime} / S_{i}$ factors through $\mathbf{P i c}^{\tau} P_{i} / S_{i} \rightarrow$ $\mathbf{P i c}^{\tau} P_{i}^{\prime} / S_{i}$, and the kernel of the latter homomorphism contains $H_{i}$. As a conclusion, the action of $H_{i}$ on $L_{i}^{\prime}$ and its pullback $L_{i}^{\dagger}$ is linearizable. In particular, we can apply this to the family $\left\{{ }_{n} T_{Y, i}\right\}$ of $n$-torsion subgroups of $T_{Y, i}$. Since this family is schematically dense ([SGA3, IX.4]) in $T_{Y, i}$, the action of $T_{Y, i}$ on $L_{i}^{\dagger}$ is linearizable.

The same argument works for the bigger torus $T_{i}$. The $T_{i}$-linearization is induced by the $T_{Y, i}$-linearization but now we can make it canonical: defining $\widetilde{P}_{i}$ to be a disjoint union of $X / Y$ copies of $P_{i}^{\dagger}$, on for the pullback $\widetilde{P}_{i}$ we obtain a canonical $T_{i}$-linearization. We have $\left(P_{i}, L_{i}\right)=\left(\widetilde{P}_{i}, \widetilde{L}_{i}\right) / X$. Because sheaf $\widetilde{L}_{i}$ is ample, $\widetilde{P}_{i}$ is identified with a Proj of the graded algebra $\mathcal{O}_{S_{i}}$-algebra $R\left(\widetilde{L}_{i}\right)=\oplus_{d \geq 0} f \widetilde{L}_{i}^{d}$. This algebra is graded by $\mathbb{Z}_{\geq 0}$ and the $T_{i}$-linearization provides an additional grading by $X$ as this algebra splits into the eigenspaces. Finally, the actions of $T$ and $X$ on $\widetilde{L}_{i}$ commute in the way that is claimed in 
the formulation of the theorem because that is how the actions of $X$ and $T$ on the algebra $R\left(\widetilde{L}_{i}\right)$ commute: $x \in X$ takes the $x^{\prime}$-eigenspace to the $\left(x+x^{\prime}\right)$ eigenspace.

This solves the problem locally. Now we want to glue $\widetilde{P}_{i}$ into an $X$-torsor $\widetilde{P}$ over $P$. Without the $T$-action, if we just had $\widetilde{P}_{i}$ 's, we would proceed in the following way. We would glue $\widetilde{P}_{i}$ 's on the "intersections" $S_{i} \times \underset{S}{\times} S_{j}$, and we would have a choice: we could change an identification by an element $x_{i j} \in X$. But then on the triple "intersections" we may run into the incompatibility problem. Exactly as in the exact sequence in the proof of 4.2.5 there would be an obstruction in $H_{\text {fppf }}^{2}(S, X)$ which has to vanish in order for the gluing to exist. In the presence of the $T$-action, however, this obstruction is zero. Because each algebra $R\left(\widetilde{L}_{i}\right)$ is $X$-linearized, on the "intersections" they glue in a canonical way, according to the $X$-eigenspaces. This completes the proof of the theorem.

COROLlary 4.3 .4 (of the proof). The connected components of $\widetilde{P}$ are parameterized by $X / Y$ which is the Cartier dual of the kernel of the polarization morphism $\lambda(L): T \rightarrow \mathbf{P i c}_{P / S}$.

Remark 4.3.5. Note that the actions of $T$ and $X$ on $\widetilde{L}$ combine into an action of the infinite Heisenberg group $\mathcal{G}=\mathbb{G}_{m, S} \underset{S}{\times} \underset{S}{\times} X$ with the following group law: $\mathbb{G}_{m}$ is the center, and if $a$ and $x$ are functorial points of $T$ and $X$ (i.e. sections after a base change $S^{\prime} / S$ ), then

$$
T_{a}^{*} S_{x}^{*}=x(a) \cdot S_{x}^{*} T_{a}^{*} .
$$

The surprising element here is that we are doing in Zariski topology things that normally only work in the classical topology over $\mathbb{C}$.

\section{Stable semiabelic varieties and pairs}

We are now going to classify stable semiabelic varieties and pairs over an algebraically closed field $k$ in the remaining cases: when there is no linearization on $L$ and also when $G$ has a nontrivial abelian part. We will also consider the case of a quotient field of a complete normal ring.

5.1. (Co) sheaves on general cell complexes. Let $\Delta=\widetilde{\Delta} / X=\Delta^{\dagger} / Y=$ $\Delta^{\ddagger} / \pi_{1}(\Delta)$ be a complex referenced by $\bar{X}_{\mathbb{R}} / Y$. Let $\left\{\delta_{i}, i \in \Delta_{\min }\right\}$ be the minimal order cells under the reverse to inclusion, and choose their representatives $\widetilde{\delta}_{i}, \delta_{i}^{\dagger}, \delta_{i}^{\ddagger}$ in the complexes $\widetilde{\Delta}, \Delta^{\dagger}, \Delta^{\ddagger}$ resp. These latter cells are either lattice polytopes or pullbacks of lattice polytopes under a projection to a lower dimension. 
We will go through the definition in the case of the cover $\Delta^{\dagger}$. The other two cases, which will also be useful, are obtained by replacing the group $Y$ everywhere formally by $X, \pi_{1}(\Delta)$ resp. For simplicity, we will only consider the case when $\Delta^{\dagger}$ is a join semilattice; i.e., intersection of two cells is again a single cell.

To define (co)chain groups $C_{p}$ and $C^{p}$ consider the nonempty intersections

$$
\delta_{\left[i_{0}\left|y_{1} \delta_{i_{1}}\right| \ldots \mid y_{p} \delta_{i_{p}}\right]}=\delta_{i_{0}} \cap y_{1} . \delta_{i_{1}} \cap \ldots y_{p} . \delta_{i_{p}}
$$

such that we do not have both $i_{k-1}=i_{k}$ and $y_{k}=0$. For each of these we can take the groups $\mathbb{X}_{\delta}, X_{\delta}, \operatorname{Fun}(X \cap \delta, \mathbb{Z}), \operatorname{Fun}(C \cap \delta)$ and define $C_{p}(\Delta, G)$ for the cosheaf $\underline{\mathbb{X}}, \underline{X}, \underline{\text { Fun }}$ resp. as the direct sum of these. The differential $C_{p} \rightarrow C_{p-1}$ is defined as $\sum(-1)^{k} d_{k}$, where

$$
d_{k}: G_{\left[i_{0}\left|y_{1} \delta_{i_{1}}\right| \ldots \mid y_{p} \delta_{i_{p}}\right]} \rightarrow G_{\left[i_{0}\left|y_{1} \delta_{i_{1}}\right| \ldots y_{k} \hat{\delta}_{i_{k}} \ldots \mid y_{p} \delta_{i_{p}}\right]}
$$

is the natural restriction homomorphism if $k \neq 0$; for $k=0$, it is the composition of restriction and the homomorphism

$$
S_{-y_{1}}: G_{\left[y_{1} \delta_{i_{1}}\left|y_{2} \delta_{i_{2}}\right| \ldots \mid y_{p} \delta_{i_{p}}\right]} \rightarrow G_{\left[\delta_{i_{1}}\left|y_{2} \delta_{i_{2}}\right| \ldots \mid y_{p} \delta_{i_{p}}\right]}
$$

given by the group action. We give the structure of the $Y$-module on the above groups in the following way:

1. on $\operatorname{Fun}(X, \mathbb{Z}): y \cdot 1_{x}=1_{x+y}$,

2. on $\mathbb{X}: y \cdot(d, x)=(d, x+d y)$,

3. on $X: y \cdot x=x$, i.e. a trivial action.

As before, we define in this more general situation:

1. groups of cochains $C^{p}(\widehat{G})=\operatorname{Hom}\left(C_{p}(G), \mathbb{G}_{m}(S)\right.$,

2. (co)homologies,

3. $\mathbb{K}$ and $\widehat{\mathbb{K}}$,

4. complexes $\mathbb{M}_{*}=\operatorname{Cone}\left(C_{*}(\underline{\text { Fun }}) \rightarrow C_{*}(\underline{\mathbb{X}})\right)$ and $\widehat{\mathbb{M}}^{*}=\operatorname{Cone}\left(C^{*}(\underline{\widehat{\text { Fun }}}) \rightarrow\right.$ $\left.C^{*}(\underline{\widehat{\mathbb{X}}})\right)$,

5. all the exact sequences of (co)sheaves and associated long exact sequences of (co)homologies as before,

6. variants of these definitions with $\underline{\text { Fun }}_{\geq 0}$.

When it makes a difference, we will specify which cover we use in the above definition by adding symbols $\dagger, \ddagger$ or $\sim$ respectively. 
Example 5.1.1. If $Y=0$, we recover the previous Cech (co)homologies on a finite complex of lattice polytopes.

Example 5.1.2. If $Y \neq 0$ but $\Delta^{\dagger}$ is a complex of lattice polytopes, we get (co)homologies of just slightly more complicated constructible (co)sheaves on the topological space $|\Delta|$.

Example 5.1.3. Consider the complex $\Delta$ consisting of one big cell $X_{\mathbb{R}} / Y$, where $Y$ is a sublattice in $X$ of finite index. In this case, we obtain the usual group (co)homologies of the corresponding $Y$-modules.

Lemma 5.1.4. 1. $H_{1}(\underline{\mathbb{X}})=(Y \otimes X) / \Lambda^{2} Y \supset(Y \otimes Y) / \Lambda^{2} Y=\Gamma^{2}(Y)$.

2. $B_{1}(\underline{\mathbb{X}}) \subset C_{1}(\underline{\mathbb{X}})=\oplus_{y \neq 0}[y] \mathbb{X}$ is the subgroup generated by the elements $\left(\left[y_{1}+y_{2}\right]-\left[y_{1}\right]-\left[y_{2}\right]\right)(0, x)$ and $\left(\left[y_{1}+y_{2}\right]-\left[y_{1}\right]-\left[y_{2}\right]\right)(1,0)-\left[y_{2}\right]\left(0, y_{1}\right)$.

Proof. From the long exact sequence of homologies for the short exact sequence

$$
0 \rightarrow \underline{X} \rightarrow \underline{\mathbb{X}} \rightarrow \mathbb{Z} \rightarrow 0
$$

we get $H_{1}(\underline{\mathbb{X}})=H_{1}(X) / H_{2}(\mathbb{Z})$. On the other hand, $H_{p}(\mathbb{Z})=H_{p}(|\Delta|, \mathbb{Z})=$ $\Lambda^{p} Y$. Also, since $X$ is a trivial $Y$-module, $H_{p}(X)=H_{p}(\mathbb{Z}) \otimes X$. This proves Part 1. Part 2 is a direct calculation.

LeMma 5.1.5. For $\Delta=X_{\mathbb{R}} / Y$ :

1. $C_{0}(\underline{\mathbb{L}})=\mathbb{L}$ is the group generated by the elements $1_{x_{1}+x_{2}}-1_{x_{1}}-1_{x_{2}}+1_{0}$.

2. $B_{0}(\underline{\mathbb{L}})$ is the subgroup of $\mathbb{L}$ generated by the elements

$$
\left(1_{x_{1}+x_{2}+y}-1_{x_{1}+y}-1_{x_{2}+y}+1_{y}\right)-\left(1_{x_{1}+x_{2}}-1_{x_{1}}-1_{x_{2}}+1_{0}\right) .
$$

Assume in addition that $Y=X$. Then:

1. $H_{0}(\underline{\mathbb{L}})=C_{0} / B_{0}(\underline{\mathbb{L}})$ is the lattice of dimension $r(r+1) / 2$ isomorphic to Sym $^{2} X$ via $1_{x_{1}+x_{2}}-1_{x_{1}}-1_{x_{2}}+1_{0} \mapsto x_{1} \otimes x_{2}$. The dual to it is the lattice of homogeneous quadratic functions on $X$.

2. $C_{0}(\underline{\text { Fun }}) / B_{0}(\underline{\mathbb{L}})$ is the lattice of dimension $r(r+1) / 2+(r+1)$. The dual to it is the lattice of nonhomogeneous quadratic functions on $X$.

Proof. This is obtained directly from definitions.

5.2. Linearized varieties with a nontrivial abelian part.

LEMma 5.2.1. Let $(P, L)$ be an irreducible variety together with an action of a semiabelian variety $G, 1 \rightarrow T \rightarrow G \rightarrow A \rightarrow 0$, such that the $T$-action on $L$ is linearized and the condition on orbits is satisfied. Then $P$ is fibered 
over A (more precisely, over an A-torsor), all fibers are isomorphic and the normalization of each is an irreducible projective toric variety with a linearized line bundle corresponding to a lattice polytope $\delta \subset X_{\mathbb{R}}$.

Proof. Pick an arbitrary point $p$ in the interior of the main orbit and let $P_{0}$ be the closure of the orbit $T p$. For each coset $T_{a}$ of $T \subset G$ the closure of $T_{a} p$ is a subvariety isomorphic to $P_{0}$. It is clear that $P_{0}$ satisfies our condition on the orbits as well, for the $T$-action, so that its normalization together with the pullback of $L$ is a projective toric variety corresponding to a lattice polytope $\delta \subset X_{\mathbb{R}}$. The 0 -dimensional orbits of $T \curvearrowright P_{0}$ correspond to the vertices of this polytope. If $q_{x}$ is one of these orbits then by our condition on the orbits, $G q_{x}=A q_{x} \simeq A$. The restriction of $L$ to $A q_{x}$ is $T$-linearized and is of weight $x$; therefore for $x \neq y$ one has $A q_{x} \cap A q_{y}=\emptyset$. This implies that different varieties $P_{a}$ do not intersect and that they define a fibration of $P$ over $A$.

Lemma 5.2.2. Let $(P, L)$ be a stable semiabelic variety with a T-linearized line bundle. Then every irreducible component of $P$ is normal and is also a stable semiabelic variety.

Proof. It is clear for any point $p \in P$ that the above fibration on the irreducible components defines a fibration of a neighborhood with isomorphic slices. The seminormality of $P$ implies the seminormality of each of these slices, and, by our results in the toric case, the normality of each irreducible component.

Recall that the semiabelian variety $G$ is defined, via the negative of pushout, by a homomorphism $c: X \rightarrow A^{t}$. If $\mathcal{O}_{x}=c(x)$ is the corresponding rigidified sheaf then

$$
G=\operatorname{Spec}_{A} \oplus_{x \in X} \mathcal{O}_{x} .
$$

Definition 5.2.3. Let $\mathcal{M}$ be an ample invertible sheaf on $A$. For $\chi=$ $(d, x) \in \mathbb{X}$ we will denote by $\mathcal{M}_{\chi}$ the sheaf $\mathcal{M}^{d} \otimes \mathcal{O}_{x}$ rigidified at the origin.

Lemma 5.2.4. Let $(P, L)$ be an irreducible semiabelic variety with a linearized line bundle. Fix a point $p \in P$ in the main G-orbit. With this choice, $P$ can be identified with the variety

$$
\operatorname{Proj}_{A} \oplus_{\chi \in \text { Cone } \delta} \mathcal{M}_{\chi}
$$

and $L$ with the sheaf $\mathcal{O}(1)$.

Proof. With the fixed projection $f: P \rightarrow A$ consider the $\mathcal{O}_{A}$-algebra $\mathcal{A}=\oplus_{d \geq 0} L^{d}$. Since the fibers of $f$ are irreducible stable toric varieties, we know that the higher direct images $R^{i} f_{*} L^{d}$ are zero, and the algebra $\mathcal{A}$ is locally free. The $T$-action on $L$ defines a splitting of $\mathcal{A}$ into a direct sum of eigenspaces, and since on each $f^{-1}(a)$ the eigenspaces are 1-dimensional, these 
$\mathcal{O}_{A}$-sheaves are invertible. Say, the $(1, x)$-eigenspace is $\mathcal{M} \otimes \mathcal{O}_{x}$ for some $\mathcal{M}$. Then the prescribed form is dictated by the fact that $\mathcal{A}$ is an algebra and $P$ has $G$-action. Finally, $\mathcal{M}$ has to be ample since from the growth of $h^{0}\left(L^{d}\right)$ we see that $h^{0}\left(\mathcal{M}^{d}\right)$ has to grow as $g^{d}, g=\operatorname{dim} A$, and on an abelian variety a big sheaf is ample. Clearly, $(P, L)=(\operatorname{Proj} \mathcal{A}, \mathcal{O}(1))$.

Definition 5.2.5. We will denote the polarized variety in this standard form by $(P, L)[\delta, c, \mathcal{M}]$.

Lemma 5.2.6. Let $(P, L)=(P, L)[\delta, c, \mathcal{M}]$. Then $H^{p}(P, L)=0$ for $p>0$ and there is a canonical isomorphism

$$
H^{0}(P, L)=\oplus_{x \in X \cap \delta} H^{0}\left(A, \mathcal{M}_{(1, x)}\right) .
$$

Proof. For the global sections this is obvious from the above, since $H^{0}(P, L)=H^{0}\left(A, f_{*} L\right)$. The higher direct images $R^{p} f_{*} L$ vanish since we have vanishing on the fibers which are projective toric varieties. Therefore, $H^{p}(P, L)=H^{p}\left(A, f_{*} L\right)$ and these are zero since higher cohomologies of ample sheaves on abelian varieties vanish.

TheOREM 5.2.7. Let $\theta \in H^{0}(P, L)$ be a section corresponding to a divisor $\Theta$. Then $(P, \Theta)$ is a stable semiabelic pair if and only if the homogeneous components for the $x \in \operatorname{Vert} \delta$ are not zero.

Proof. Indeed, these homogeneous components define the restrictions of $\Theta$ to each minimal $G$-orbit.

Definition 5.2.8. We will denote the subset of $H^{0}\left(A, \mathcal{M}_{(1, x)}\right)$ with the above condition by $\widehat{\mathrm{Fun}}_{\geq 0, \delta}$.

5.3. Arbitrary polarized SSAVs. Now, let $(P, L)$ be an arbitrary polarized stable semiabelic variety. By the main result of Section 4 the pair $(P, L)$ is equivalent to a pair $(\widetilde{P}, \widetilde{L})$ together with an $X$-action, where $\widetilde{L}$ is $T$-linearized but the scheme $\widetilde{P}$ is only locally of finite type over $k$. For schemes which are locally of finite type over a field the seminormality is a formal property, and it is certainly preserved under an étale morphism. Therefore, any finite union of irreducible components of $\widetilde{P}$ together with the restriction of $\widetilde{L}$ is a linearized polarized stable semiabelic variety. Therefore, the pair $(\widetilde{P}, \widetilde{L})$ defines a complex $\widetilde{\Delta}$ of lattice polytopes which is locally of finite type, together with an $X$-action compatible with the reference map $\tilde{\rho}:|\widetilde{\Delta}| \rightarrow X_{\mathbb{R}}$.

The $X$-action on $\widetilde{\Delta}$ is not properly discontinuous in the order topology. However, it is if we work instead with the classical topology on $|\widetilde{\Delta}|$. Introduce the complex $\Delta$ as the quotient $\widetilde{\Delta} / X$. If $\Delta^{\dagger}$ is a connected component of $\widetilde{\Delta}$ and $Y \subset X$ the subgroup leaving it invariant then $\Delta=\Delta^{\dagger} / Y$. The complex $\Delta$ is naturally referenced with a map to $\bar{X}_{\mathbb{R}} / Y$, where $\bar{X}$ is an $X$-torsor, i.e., 
the group $\mathbb{Z}^{r}$ but without the origin fixed. Moreover, for every $X$-linearized cosheaf $G$, resp., sheaf $F$, on $|\widetilde{\Delta}|$ we can consider the corresponding quotient cosheaf, resp., sheaf, by the free and properly discontinuous $X$-action. We will denote these quotient cosheaves and sheaves on $|\Delta|$ by the same letters.

Definition 5.3.1. We introduce one more complex $\Delta^{\ddagger}$, the universal cover of $\Delta^{\dagger}$. It comes with the reference map to $X_{\mathbb{R}}$ and corresponds to a polarized scheme $\left(P^{\ddagger}, L^{\ddagger}\right)$ locally of finite type such that $(P, L)=\left(P^{\ddagger}, L^{\ddagger}\right) / \pi_{1}(|\Delta|)$. We will assume for simplicity that the complex $\Delta^{\ddagger}$ is a poset; i.e., the intersection of two polytopes is again a polytope (the general case is only a little harder).

Definition 5.3.2. Pick an arbitrary point $p$ in a minimal orbit $A p \subset P$. For each cycle in $\pi_{1}(|\Delta|)$ we can trace a cycle of $T$-orbits on our variety along the fibrations to come back to another point which is a shift of $p$ by the $A$-action. This way, the variety $P$ defines a homomorphism from $\pi_{1} \Delta$ to $A$ which we will denote by $c^{t}$.

5.3.3. Choose a system $\left\{\delta_{i}, \mid i \in I\right\}$ of representatives in $\Delta^{\dagger}$ of all the minimal cells in $\Delta$. Also, choose a point in $P^{\ddagger}$. With these choices made, we can identify the subvarieties corresponding to $\delta_{i}$ with the standard polarized varieties $P\left[\delta_{i}, c, \mathcal{M}\right]$ for the same $c, \mathcal{M}$. We can do so uniquely: since there are no cycles in $\Delta^{\ddagger}$, any tracing out gives the same origin in $A$. All other irreducible components of $P^{\ddagger}$ correspond to the cells $z . \delta_{i}$ for some $z \in \pi_{1} \Delta$. Hence, we can also identify all the irreducible components of $P^{\ddagger}$ with the translations of these standard varieties. To give the variety $(P, L)$ is the same as to give the scheme $\left(P^{\ddagger}, L^{\ddagger}\right)$ together with the action of $\pi_{1} \Delta$. Therefore, we see that we have to describe the gluing of the shifted algebras $T_{c^{t} z}^{*} R_{i}$ for the cells $z . \delta_{i}$ on the intersections. To this end, we see first of all that these corresponding invertible sheaves have to be isomorphic. This translates to the condition that

$$
\lambda(\mathcal{M}) \circ c^{t}=c \circ i: \pi_{1} \rightarrow A^{t}
$$

where we denoted by $i$ the projection $\pi_{1} \Delta \rightarrow X$ given by the reference map $\Delta^{\ddagger} \rightarrow X_{\mathbb{R}}$.

Definition 5.3.4. Assuming $c, c^{t}$ and $\mathcal{M}$ given, we will define the cochain groups $C_{\ddagger}^{p}(\Delta, \underline{\mathbb{X}})$ for $p=0,1$ as functions on $C_{p}^{\ddagger}(\Delta, \underline{\mathbb{X}})$ linear in $\mathbb{X}$, where the function on

1. [] $\chi$ takes values in $\mathbb{G}_{m}(k)$,

2. $[z] \chi$ takes values in the $\mathbb{G}_{m}(k)$-torsor $\operatorname{Iso}\left(\mathcal{M}_{\chi}, T_{-c^{t} z^{*}} \mathcal{M}_{z \cdot \chi}\right)$ which is the same as the fiber $\mathcal{M}_{\chi}^{-1}\left(c^{t} z\right)$ without the zero.

Lemma 5.3.5. The functions on $B_{1}(\underline{\mathbb{X}})$ take value canonically in $\mathbb{G}_{m}(k)$. 
Proof. We have to check this in two cases: $\chi=(0, x)$ and $\chi=(1,0)$. In the first case this reduces to a canonical identification of $\mathbb{G}_{m}$-torsors $\mathcal{O}_{x}\left(c^{t} z_{1}+\right.$ $\left.c^{t} z_{2}\right)=\mathcal{O}_{x}\left(c^{t} z_{1}\right) \otimes \mathcal{O}_{x}\left(c^{t} z_{2}\right)$ which is a consequence of the so-called bi-extension structure on the Poincaré bundle on $A \times A^{t}$. In the second case this reduces to the identity

$$
\mathcal{M}\left(c^{t} z_{1}+c^{t} z_{2}\right) \otimes \mathcal{M}\left(c^{t} z_{1}\right)^{-1} \otimes \mathcal{M}\left(c^{t} z_{2}\right)^{-1}=\mathcal{O}_{i z_{1}}\left(c^{t} z_{2}\right)
$$

which is to say that the symmetric bi-extension on $A \times A$ coincides with the pullback of the Poincaré bi-extension on $A \times A^{t}$ via (id, $\lambda(\mathcal{M})$ ). This follows from our commuting condition 3 .

Definition 5.3.6. We will denote by $Z^{1}(\underline{\widehat{\mathbb{X}}})$ the functions on $C_{1} / B_{1}(\underline{\mathbb{X}})$, i.e. the functions on $C_{1}$ that are identically 1 on $B_{1}$.

Definition 5.3.7. A framing for a polarized stable semiabelic variety will have the following characteristics:

1. isomorphism $X \stackrel{\sim}{\rightarrow} \mathbb{Z}^{r}$,

2. the connected component $\Delta^{\dagger}$,

3. choice of the representatives for the minimal cells $\delta_{i}$,

4. projections to $A$ of the varieties for these selected cells.

Under minor modifications of the definitions of cohomologies, Theorem 2.4.11 holds literally:

TheOrem 5.3.8. The groupoid $M^{\mathrm{fr}}\left[\Delta, c, c^{t}, \mathcal{M}\right](k)$ is equivalent to

$$
\left[Z_{\ddagger}^{1}(\Delta, \underline{\widehat{\mathbb{X}}}) / C_{\ddagger}^{0}(\Delta, \underline{\widehat{\mathbb{X}}})\right] .
$$

Proof. Indeed, elements of $Z^{1}$ describe the compatible gluings, and $C^{0}$ describes the choices of an origin in each of the $P\left[\delta_{i}, c, \mathcal{M}\right]$ that do not change the projection to $A$.

Concerning the singularities, we have the following, which is the same as the toric case.

THEOREM 5.3.9. Let $(P, L)$ be a stable semiabelic variety corresponding to a complex $\Delta$ plus all the other data. If $\Delta$ is locally $\mathrm{CM}$ (for example $|\Delta|$ is a manifold with a boundary) then $P$ is CM.

Proof. For the schemes which are locally of finite type over a field, the property of being Cohen-Macaulay is a formal property. Therefore, we can go over to the étale cover $\widetilde{P}$ for this question. Additionally, for any point $p \in P$ 
the completion of the local ring splits as the direct product of the abelian part (smooth) and the toric part, the latter being the same as for a stable toric variety for the same complex. The result now follows.

\subsection{Arbitrary stable semiabelic pairs.}

THEOREM 5.4.1. For any polarized stable semiabelic variety $(P, L)$ one has $H^{p}\left(P, L^{d}\right)=0$ for $p, d \geq 1$.

Proof. The irreducible linearized case was established in Theorem 5.2.6.

It is sufficient to prove the statement for a finite cover with the pullback of $L$. This finite cover can be chosen to be again an SSAV but with the condition that the intersections of irreducible components are irreducible, i.e., that intersection of two polytopes in $\Delta$ is a single polytope. As in the proof for the toric case, consider the cover of $P$ by the varieties $P_{i_{0}}$ corresponding to $\mathcal{U}_{\text {min }}$. Denoting $P_{i_{0} i_{1}}=P_{i_{0}} \cap P_{i_{1}}$ and so forth (they are all also SSAVs of lower dimension), we get a resolution of $\mathcal{O}_{P}$ by the complex having $\oplus \mathcal{O}_{P} i_{0} \ldots i_{p}$ in degree $p$. Computing the hypercohomologies of this complex and using induction on the dimension we complete the proof.

Definition 5.4.2. Keeping the analogy with the linearized toric case, for an SSAV described in the previous section we define

1. $C^{0}\left(\Delta, \widehat{\operatorname{Fun}}_{\geq 0}\right)=\oplus \widehat{\operatorname{Fun}}_{\geq 0, i}$ (see Definition 5.2.8),

2. $\widehat{\mathbb{M}}^{0}(\Delta)=C^{0}(\Delta, \underline{\widehat{\mathbb{X}}})$,

3. $\widehat{\mathbb{M}}^{1}(\Delta)=C^{0}\left(\Delta, \widehat{\widehat{\text { fun }}}_{\geq 0}\right) \oplus C^{1}(\Delta, \underline{\widehat{\mathbb{X}}})$.

Note that the homogeneous part $\tau_{i_{1} i_{0}}^{[z] \chi}$ of $\tau \in C^{1}(\Delta, \widehat{\mathbb{X}})$ which is the value on $[z] \chi$ is an element of Iso $\left(\mathcal{M}_{\chi}, T_{-{ }^{t} z^{*}} \mathcal{M}_{z \cdot \chi}\right)$ and it induces an isomorphism from $H^{0}\left(P_{i_{0}}, \mathcal{M}_{\chi}\right)$ to $H^{0}\left(P_{i_{1}}, T_{-c^{t} z}^{*} \mathcal{M}_{z \cdot \chi}\right)=T_{-c^{t} z} H^{0}\left(P_{i_{1}}, \mathcal{M}_{z \cdot \chi}\right)$. We will denote by $Z^{1}\left(\widehat{\mathbb{M}}^{*}\right)$ the set of pairs $(f, \tau) \in \widehat{\mathbb{M}}^{1}(\Delta)$ such that $f_{i_{1}}=T_{-c^{t} z}\left(\tau_{i_{1} i_{0}}\left(f_{i_{0}}\right)\right)$.

With these straightforward generalizations, Theorem 2.6 .8 remains literally true:

TheOrem 5.4.3. The groupoid $\operatorname{MP}^{\mathrm{fr}}\left[\Delta, c, c^{t}, \mathcal{M}\right](k)$ is equivalent to

$$
\left[Z_{\ddagger}^{1}\left(\Delta, \widehat{\mathbb{M}}^{*}\right) / \widehat{\mathbb{M}}_{\ddagger}^{0}(\Delta)\right] .
$$

Proof. With the previous theorem at hand, the proof goes exactly as in the polarized toric case. 
5.4.4. It is now time to describe the "unframed" groupoid of all stable semiabelic pairs. We will start with a complex $\Delta$ referenced by $\bar{X}_{\mathbb{R}}$ and a fixed abelian variety $A$ with a polarization $\lambda$. First, we have the space $\operatorname{Hom}^{\prime}\left(\pi_{1} \Delta \times\right.$ $\left.X, A \times A^{t}\right)$ of homomorphisms $\left(c^{t}, c\right)$ satisfying the commuting condition 3 . Let $\operatorname{Pic}^{\lambda} A$ be the component of Pic $A$ containing the sheaves with $\lambda(\mathcal{M})=\lambda$. Then over this space we have the family of $Z^{1}\left(\Delta, \widehat{\mathbb{M}}^{*}\right)$. To arrive at $\operatorname{MP}[\Delta, A, \lambda]$ we have to divide this family by three consecutive equivalence relations:

1. by the action of $\widehat{\mathbb{M}}^{0}(\Delta)$ - this one is the same for each fiber,

2. by the action of $A$ which corresponds to a change of origin in $A$,

3. and, finally by the groups of symmetries of the complex $\Delta$ and the pair $(A, \lambda)$.

Here, the first one is given by a torus action but the action is proper with finite stabilizers, by the semigroup Lemma 2.9.6. The second one is a quotient by a proper action of a proper group. And finally the third one is a quotient by a finite group.

Moreover, we can do the same over a stack of all abelian varieties of dimension $g$ and a polarization of degree $d$. By the result of Keel-Mori [KM] this gives a separated stack $\operatorname{MP}[\Delta, g, d]$ over $\mathbb{Z}$ which admits a coarse moduli space as a separated algebraic space.

5.5. Stable semiabelic pairs over $\mathbb{C}$. To describe stable semiabelic varieties and pairs over an arbitrary closed field we used a complex of lattice polytopes $\Delta^{\prime}$ (we called it simply $\Delta$ before to simplify notation) referenced by $\bar{X}_{\mathbb{R}}^{\prime} / Y^{\prime}$, where $X^{\prime} \simeq \mathbb{Z}^{r}, r \leq g$, was the character group of the toric part. The formulas were a little complicated since they involved cohomologies with values in certain $\mathbb{G}_{m}$-torsors.

A variety or a pair over the field of complex numbers can be described much more directly, by using a complex $\Delta$ with cells of full dimension which are not necessarily polytopal. The reason for this is that a $g$-dimensional semiabelian variety $G$ can be, noncanonically, represented as $\left(\mathbb{C}^{*}\right)^{g} / Y$ and so a variety $P$ with $G$-action can be formally considered to be "toric" (this action is not algebraic, of course). An abelian variety corresponds in this way to one big cell $\bar{X} / Y$. A general semiabelic variety corresponds to a general cell complex. As in the toric case, a variety and its complex are related through the moment map Mom : $P(\mathbb{C}) \rightarrow|\Delta|$. A fiber of this map over a point in the interior of a $k$-dimensional cell $\delta$ is isomorphic to $\left(S^{1}\right)^{k}$.

It turns out that the classification theorem for stable toric varieties and pairs hold here verbatim, with one small difference: the varieties and pairs are classified not by entire groups $H^{1}(\underline{\widehat{\mathbb{X}}})$, resp. $H^{1}\left(\widehat{\mathbb{M}}^{*}\right)$, but by open subsets of points that satisfy the Riemann positivity condition. The dimension of the 
corresponding stratum is, therefore, still $h^{1}(\underline{\widehat{\mathbb{X}}})$, resp. $h^{1}\left(\widehat{\mathbb{M}}^{*}\right)$. Since our strata were constructed over $\mathbb{Z}$ and the dimension does not depend on a particular field, the same dimension formula holds over an arbitrary field $k$.

5.5.1. We will start with a single abelian variety $A=\mathbb{C}^{g} / \mathbb{Z}^{2 g}$ with a polarization $\lambda$ of type $\mathbb{Z}_{d_{1}} \times \ldots \mathbb{Z}_{d_{g}}$. Recall from the classical theory that via exponentiation $\exp (2 \pi i \cdot)$ of half of the periods $(A, \lambda)$ can be represented in the following way:

1. $A=T / i(Y), T=\left(\mathbb{C}^{*}\right)^{g}, Y=\mathbb{Z}^{g}$. If we denote by $X$ the group of characters of $T$ then we get a bilinear function $b: Y \times X \rightarrow \mathbb{C}^{*}, b(y, x)=$ $x(y)$;

2. $Y \subset X$ is a sublattice with $X / Y=\mathbb{Z}_{d_{1}} \times \ldots \mathbb{Z}_{d_{g}}$ such that $\left.b\right|_{Y \times Y}$ : $Y \times Y \rightarrow \mathbb{C}^{*}$ is symmetric and positive definite in the following sense: $|b(y, y)|<1$ for all $y \neq 0$.

There is a group $\operatorname{Sp}_{2 g}\left(\operatorname{diag}\left(d_{1}, \ldots, d_{g}\right), \mathbb{Z}\right)$ acting properly discontinuously in classical topology on the set of all such representations, and the quotient is the component of $\mathrm{A}_{g, d}$ corresponding to polarizations of type $\left(d_{1}, \ldots, d_{g}\right)$. The subgroup of $\operatorname{Sp}_{2 g}\left(\operatorname{diag}\left(d_{1}, \ldots, d_{g}\right), \mathbb{Z}\right)$ that preserves the structure of a "toric variety" on $A$, i.e. the presentation $A=\widehat{X} / Y$ is a subgroup $\operatorname{GL}(X, Y) \subset$ $\mathrm{GL}(X)$ that sends $Y$ to itself. The remaining equivalence relation by which we have to divide in order to arrive at the quotient by $\operatorname{Sp}\left(\operatorname{diag}\left(d_{1}, \ldots, d_{g}\right), \mathbb{Z}\right)$ corresponds to a different choice of a maximal isotopic subspace in $\mathbb{Z}^{2 g}$.

The datum for an ample sheaf $L$ on $A$ with $\lambda(L)=\lambda$ is a function $a: Y \rightarrow \mathbb{C}^{*}$ such that $a\left(y_{1}+y_{2}\right)=a\left(y_{1}\right) a\left(y_{2}\right) b\left(y_{1}, y_{2}\right)$.

LEMMA 5.5.2. The datum above is precisely the same as an element of $Z^{1}\left(\bar{X}_{\mathbb{R}} / Y, \underline{\widehat{\mathbb{X}}}\right)=\operatorname{Hom}\left(C_{1} / B_{1}\left(\bar{X}_{\mathbb{R}} / Y, \underline{\mathbb{X}}\right), \mathbb{C}^{*}\right)$ whose restriction to the subgroup $\mathrm{Sym}^{2} Y$ is positive definite.

Proof. Indeed, by Lemma 5.1.4 an element of $\operatorname{Hom}\left(C_{1} / B_{1}\left(\bar{X}_{\mathbb{R}} / Y, \underline{\mathbb{X}}\right), \mathbb{C}^{*}\right)$ is a function $\tau(y, d, x)$ on $Y \times \mathbb{X}$ which is linear in $\mathbb{X}$ and which satisfies two types of relations. If we denote $\tau(y, 0, x)=b(y, x)$ and $\tau(y, 1,0)=a(y)$ then by linearity we can write $\tau(y, d, x)=b(y, x) a(y)^{d}$ and the relations then take form:

1. $b\left(y_{1}+y_{2}, x\right)=b\left(y_{1}, x\right) b\left(y_{2}, x\right)$

2. $a\left(y_{1}+y_{2}\right)=a\left(y_{1}\right) a\left(y_{2}\right) b\left(y_{1}, y_{2}\right)$,

which is exactly what we have above. 
Hence, formally, $\left(\mathbb{C}^{*}\right)^{g} \rightarrow A$ plays the role of the cover $P^{\dagger} \rightarrow P$ that we had before, and the line bundle $\left(\mathbb{C}^{*}\right)^{g} \times \mathbb{C}$ plays the role of $L^{\dagger}$ with $L=L^{\dagger} / Y$. We still have the algebra $R^{\dagger}=\mathbb{C} \oplus_{d>0} H^{0}\left(P^{\dagger},\left(L^{\dagger}\right)^{d}\right)$ and $P^{\dagger}=$ Proj $R^{\dagger}$, even though it is no longer a projective variety since $R_{+}^{\dagger}$ is not finitely generated.

Note that to classify abelian torsors we have to divide by a choice of the origin in the above representation, which is the same as dividing by the action of $C^{0}(\underline{\widehat{\mathbb{X}}})=\widehat{\mathbb{X}}=\mathbb{T}$. As a result, polarized abelian torsors over $\mathbb{C}$ are classified by an open subset of $H^{1}\left(\bar{X}_{\mathbb{R}} / Y, \underline{\widehat{\mathbb{X}}}\right)$ modulo $\operatorname{Sym}\left(\bar{X}_{\mathbb{R}} / Y\right)$, which contains $\mathrm{GL}(X, Y)$ as a subgroup of finite index. This set is precisely the set of bilinear functions $b(y, x)$ whose restriction on $Y \times Y$ is symmetric and positive definite.

Next, consider sections in $H^{0}(A, L)$. We start with functions $\zeta^{(1, x)}$ on $\left(\mathbb{C}^{*}\right)^{g} \times \mathbb{C}=\operatorname{Spec} \mathbb{C}\left[\mathbb{Z}_{\geq 0} \times X\right]$ corresponding to elements $(1, x) \in \mathbb{Z}_{\geq 0} \times X$. These are sections of $H^{0}(\widetilde{P}, \widetilde{L})=H^{0}(T, \mathcal{O})$. According to the classical theory of theta functions, a section of $H^{0}(A, L)$ can be represented as a $Y$-invariant section of $H^{0}(T, \widetilde{L})$ given by a power series

$$
\sum_{X} \xi_{x}=\sum_{X} f_{x}(1, x)=\sum_{X} f_{x} \zeta^{(1, x)}
$$

with $f_{x} \in \mathbb{C}$ satisfying $f_{x+y}=a(y) b(y, x) f_{x}$. The convergence of this power series reduces to the fact that the radius of convergence of the series $\sum \rho^{n^{2}} z^{n}$ is $+\infty$ if $|\rho|<1$.

Lemma 5.5.3. Abelic pairs over $\mathbb{C}$ with a given $T$-action are classified by an open subset in $H^{1}\left(\bar{X}_{\mathbb{R}} / Y, \widehat{\mathbb{M}}^{*}\right)=H^{0}\left(\bar{X}_{\mathbb{R}} / Y, \underline{\widehat{\mathbb{L}}}\right)$ whose restriction to $\operatorname{Sym}^{2} Y$ is positive-definite, modulo $\operatorname{Sym}\left(\bar{X}_{\mathbb{R}} / Y\right)$.

Proof. First of all, in this case $\mathbb{K}=0$, so that the exact sequence of Lemma 2.2.7. (4) gives $H^{1}\left(\bar{X}_{\mathbb{R}} / Y, \widehat{\mathbb{M}}^{*}\right)=H^{0}\left(\bar{X}_{\mathbb{R}} / Y, \underline{\mathbb{L}}\right)$. An element of $Z^{1}\left(\widehat{\mathbb{M}}^{*}\right)$ is by definition a pair $(\tau, f)$ with $\tau$ as before, satisfying $f_{x+y}=$ $\tau(y, 1, x) f_{x}$. But $\tau(y, 1, x)=\tau(y, 1,0) \tau(y, 0, x)=a(y) b(y, x)$. This proves that pairs $(A, \Theta)$ are classified by the open subset of $Z^{1}\left(\widehat{\mathbb{M}}^{*}\right)$. Dividing by the choice of the origin, i.e. by the action of $C^{0}\left(\widehat{\mathbb{M}}^{*}\right)=C^{0}(\underline{\widehat{\mathbb{X}}})=\mathbb{T}$ gives $H^{1}\left(\widehat{\mathbb{M}}^{*}\right)$. Finally, we have to divide by automorphisms of the pairs $(X, Y)$ and by the choice of origin in $X$.

5.5.4. Next, consider an (irreducible) semiabelic variety $P_{\delta^{\prime}}$ with an action by a semiabelian variety $G, 1 \rightarrow T^{\prime} \rightarrow G \rightarrow A \rightarrow 0, T^{\prime}=\widehat{X}^{\prime}, X^{\prime} \simeq \mathbb{Z}^{r}$, and with a $T^{\prime}$-linearized ample sheaf $L_{\delta^{\prime}}$. As we saw, this variety corresponds to a polytope $\delta^{\prime}$ referenced by the lattice $X^{\prime}$. Now make a choice of a decomposition $G=T / Y=\widehat{X} / Y=\left(\mathbb{C}^{*}\right)^{g} / Y$, so that the morphism $T^{\prime} \rightarrow T / Y$ extends to an injection $T^{\prime} \rightarrow T$ and, dually, to a surjection $X \rightarrow X^{\prime}$. Let $X_{1}=\operatorname{ker}\left(X \rightarrow X^{\prime}\right)$. If the sheaf $\mathcal{M}$ on the abelian part $A$ defines a polarization of type $\left(d_{1}, \ldots, d_{a}\right)$, then choose a sublattice $Y_{1} \subset X_{1}$ with $X_{1} / Y_{1}=\mathbb{Z}_{d_{1}} \times \ldots \mathbb{Z}_{d_{a}}$ and consider 
the complex $\Delta$ referenced by $X_{\mathbb{R}} / Y_{1}$ consisting of one cell $\delta / Y_{1}$, where $\delta$ is the pullback of $\delta^{\prime}$ under the projection $X_{\mathbb{R}} \rightarrow X_{\mathbb{R}}^{\prime}$. The standard exact sequence $0 \rightarrow \underline{X} \rightarrow \underline{\mathbb{X}} \rightarrow \mathbb{Z} \rightarrow 0$ together with an embedding of the constant cosheaf $Y_{1}$ into $\underline{X}$ give, as before, an embedding $\operatorname{Sym}^{2} Y_{1} \rightarrow H^{1}\left(\delta / Y_{1}, \underline{\widehat{\mathbb{X}}}\right)$.

Lemma 5.5.5. $\quad$ Polarized semiabelic varieties $(P, L)$ with linearized $L$ with a fixed T-action are classified by an open subset in $H^{1}\left(\delta / Y_{1}, \underline{\widehat{X}}\right)$ of positive-definite classes (i.e. those that restrict to positive-definite functions on $\left.\operatorname{Sym}^{2} Y_{1}\right)$, modulo the group of symmetries of $\delta / Y_{1}$. Semiabelic pairs with $(P, \Theta)$ with linearized $L=\mathcal{O}(\Theta)$ are classified by a similar quotient of an open subset in $H^{1}\left(\delta / Y_{1}, \widehat{\mathbb{M}}^{*}\right)$ of positive-definite classes.

Proof. Write $X=X_{1} \oplus X^{\prime}$. As an easy computation shows, a 1-cocycle in this case is a function $\tau\left(y, d, x_{1}, x^{\prime}\right)$ which by linearity can be written as $b\left(y, x_{1}\right) a\left(x_{1}\right)^{d} c\left(y, x^{\prime}\right)$ and functions $a, b, c$ satisfy:

1. $b: Y_{1} \times X_{1} \rightarrow \mathbb{C}^{*}$ is bilinear and its restriction to $Y_{1} \times Y_{1}$ is symmetric and positive definite,

2. $a\left(y_{1}+y_{2}\right)=a\left(y_{1}\right) a\left(y_{2}\right) b\left(y_{1}, y_{2}\right)$,

3. $c: Y_{1} \times X^{\prime} \rightarrow \mathbb{C}^{*}$ is bilinear.

The first two pieces of the data are equivalent to giving an abelian variety $A$ and an ample sheaf $\mathcal{M}$ on it. The third piece is a homomorphism $X^{\prime} \rightarrow$ $\widehat{Y}_{1}=\left(\mathbb{C}^{*}\right)^{g}$. The automorphism group of $\delta / Y_{1}$ is a finite extension of the group of linear transformations of $X$ given by arbitrary homomorphisms $\phi: X^{\prime} \rightarrow$ $X_{1}$. This means that we have to divide the set of $c$ 's by all homomorphisms obtained from $b$ via some $\phi$. Hence, the revised third piece of the data is a homomorphism $X^{\prime} \rightarrow \widehat{Y}_{1} / j\left(X_{1}\right)$. But the above is precisely $\operatorname{Pic}^{0} A$, and a homomorphism $X^{\prime} \rightarrow \operatorname{Pic}^{0} A$ is equivalent to the extension $1 \rightarrow T^{\prime} \rightarrow G \rightarrow$ $A \rightarrow 0$. Hence, a class in $H^{1}\left(\delta / Y_{1}, \widehat{\mathbb{M}}^{*}\right)$ modulo automorphisms is the same as giving the semiabelian variety $G$ and ample sheaf $\mathcal{M}$ on $A$. The rest follows easily.

We can now take up the general case. As we already know, an arbitrary polarized stable semiabelic variety (resp. pair) defines a complex $\Delta^{\prime}$ of lattice polytopes referenced by $\bar{X}_{\mathbb{R}}^{\prime} / Y^{\prime}$ (resp. a pointed complex) and a polarization of type $\left(d_{1}, \ldots d_{a}\right)$ on the abelian part $A$. Again, write $G=\widehat{X} / Y_{1}=\left(\mathbb{C}^{*}\right)^{g} / Y_{1}$ and consider

1. the complex $\Delta^{\dagger}$ which is the pullback of $\Delta^{\prime}$ under $X \rightarrow X^{\prime}$,

2. the complex $\Delta=\Delta^{\dagger} / Y_{1}$ with compact cells. It is referenced by $\bar{X}_{\mathbb{R}} / Y$, where $0 \rightarrow Y_{1} \rightarrow Y \rightarrow Y^{\prime} \rightarrow 0$ is a natural split extension.

As before, we have a natural embedding $\operatorname{Sym}^{2} Y_{1} \rightarrow H_{1}(\Delta, \underline{\mathbb{X}})$, so that we can talk about positive definite cohomology classes. 
TheOrem 5.5.6. Over $\mathbb{C}$, polarized stable semiabelic varieties of type $\Delta$ (resp. pairs of type $(\Delta, C)$ ) with a fixed T-action are classified by an open subset of positive definite classes in $H^{1}(\Delta, \widehat{\widehat{\mathbb{X}}})$ (resp. $\left.H^{1}\left((\Delta, C), \widehat{\mathbb{M}}^{*}\right)\right)$, modulo the group of symmetries of $\Delta$.

Proof. Indeed, $Z^{1}$ is precisely the gluing data for the elementary blocks that we have just considered, and the action of $C^{0}$ corresponds to changing the origins in these blocks.

5.5.7. Finally, we want to define a moment map for a stable semiabelic pair $(P, \Theta)$ corresponding to a complex $\Delta$ referenced by $\bar{X}_{\mathbb{R}} / Y$. Let $\theta \in H^{0}(P, L)$ be an equation of $\Theta$. By choosing a representation $A=(\mathbb{C} *)^{g} / Y_{1}$ as above we find covers $P^{\dagger}$ and $\widetilde{P}$ with a not necessarily algebraic toric action such that $(P, L)=\left(P^{\dagger}, L^{\dagger}\right) / Y=(\widetilde{P}, \widetilde{L}) / X$. We will work with the second cover. As above, the sheaf $\widetilde{L}$ comes with a section $\widetilde{\theta}=\sum_{X} \xi_{x}$ which is a (converging everywhere) power series. Just as in the finite case, we define

$$
\text { Mom }: \widetilde{P} \rightarrow|\widetilde{\Delta}| ; \quad p \mapsto \frac{\sum\left|\xi_{x}(p)\right|^{2} x}{\sum\left|\xi_{x}(p)\right|^{2}} .
$$

We leave it to the reader to check that:

1. The above power series is everywhere converging as well; this reduces to the fact that the power series $\sum n \rho^{n^{2}} z^{n}$ has a radius of convergence $+\infty$ if $|\rho|<1$.

2. Mom commutes with the $X$-actions on $\widetilde{P}$ and $\widetilde{\Delta}$, and hence descends to a moment map Mom $: P \rightarrow|\Delta|$.

3. A fiber over a point in the interior of a $k$-dimensional cell $\delta$ is isomorphic to $\left(S^{1}\right)^{k}$.

5.6. Mumford-Faltings-Chai's uniformization of abelian varieties.

Mumford-Faltings-Chai construction provides a uniformization of an abelian variety over a quotient field $\mathcal{K}$ of a normal excellent ring $\mathcal{R}$ complete with respect to an ideal $I$. We would now like to show that the data describing this uniformization are formally the same data as those for a stable semiabelic variety of type $\left[c, c^{t}, \mathcal{M}\right]$. These data contain an element of the group $Z^{1}(\Delta, \underline{\widehat{\mathbb{X}}})$ as defined in Section 5.3. The complex $\Delta$ consists of one cell $X_{\mathbb{R}} / Y$, where $X=X^{\prime}$ is the group of characters for the toric part of the central fiber. The situation, therefore, is very similar to the case of field $\mathbb{C}$.

Theorem 5.6.1 (Mumford-Faltings-Chai). There is a 1-to-1 correspondence between the following:

1. A semiabelian scheme over $\mathcal{R}$ together with an ample sheaf such that 
(a) the generic fiber over $\mathcal{K}$ is abelian,

(b) the special fiber over $\mathcal{R} / I$ is semiabelian with a split torus part.

2. The degeneration data which contain an element

$$
\tau \in Z^{1}\left(X_{\mathbb{R}} / Y, \widehat{\mathbb{X}}\left[c, c^{t}, A, \mathcal{M}\right]\right)
$$

as defined in Section 5.3, with $c, c^{t}, A, \mathcal{M}$ over $\mathcal{R}$, and with the positivity condition: the projection of $\tau$ to the functions on $\operatorname{Sym}^{2} Y$ has to be positive definite with respect to the powers of $I$.

Proof. This is the reformulation of the content of Chapters II and III of [FC]; see especially Theorems II.4.1 and II.5.1. The functions $a(y)$ and $\psi(y)$, there, in our notation are the functions on $[y](1,0)$, and the functions $b(y, x)$ and $\tau(y, x)$ are the functions on $[y](0, x)$.

Remark 5.6.2. Note that one has an element of $Z^{1}$ and not of $H^{1}$ because we look for an abelian variety over $\mathcal{K}$ and not a torsor.

5.7. One-parameter families of stable semiabelic pairs. In the next three sections we shall follow very closely the linearized toric case considered in 2.8, $2.9,2.10$ and do most things by analogy. We will also use the same or very similar notation. Hence, in this section $\mathcal{R}$ denotes a DVR with the quotient field $\mathcal{K}$ etc. $\left(P_{\eta}, \Theta_{\eta}\right)$ is a stable semiabelic pair with the action of a semiabelian group scheme $G_{\eta}$ over $\mathcal{K}$.

Reduction Step 1. First, we recall that by the stable reduction theorem, after a finite base change $G_{\eta}$ can be extended to a semiabelian group scheme $G$ over $\mathcal{R}$. After an étale base change we can assume that the toric part of the central fiber is split with a character group $X \simeq \mathbb{Z}^{r}$.

One important difference from the purely toric case is this: if the toric parts of $G_{\eta}$ and $G_{0}$ have different dimensions, i.e. $G_{\eta}$ degenerates, then we assume $\mathcal{R}$ to be complete. This does not hurt our chances of proving properness of the moduli space: in the valuative criterion of properness the valuation rings considered can be assumed to be complete DVRs, in fact, even with an algebraically closed residue field.

THEOREM 5.7.1. After a finite, possibly ramified, base change the pair $\left(P_{\eta}, \Theta_{\eta}\right)$ can be extended to a family $(P, \Theta)$ of stable semiabelic pairs over $\mathcal{R}$. Moreover, such an extension is unique, up to an isomorphism.

Proof. We will first prove this theorem in two special cases:

1. when $G_{\eta}$ does not degenerate,

2. when $G_{\eta}$ is abelian (the case considered in the Mumford-Faltings-Chai uniformization). 
After this is done, the general case is obtained as a straightforward combination of the two.

Case 1 . In this case $G$ is a global over $\mathcal{R}$ extension of a split torus $T$ by an abelian scheme $A$.

As in Section 2.8, consider an even more special case first: when $\left(P_{\eta}, \Theta_{\eta}\right)$ $\otimes \mathcal{K}$ corresponds to a single polytope $Q \subset X_{\mathbb{R}}$. After an étale base change we can put $\left(P_{\eta}, \Theta_{\eta}\right)$ in the standard form: $P_{\eta}\left[\delta, \mathcal{M}_{\eta}\right]$. By properness of $A^{t}$ and $\operatorname{Pic}_{A / S}^{\lambda}$ over $S$ the sheaf $\mathcal{M}_{\eta}$ can be extended to an ample sheaf $\mathcal{M}$ over $A$. We have a $\mathcal{K}$-algebra

$$
R_{\eta}=\oplus H^{0}\left(A, \mathcal{M}_{\chi}\right) \underset{\mathcal{R}}{\otimes} \mathcal{K}
$$

For each $x \in X$ we have an invertible $\mathcal{O}_{A}$-module $\mathcal{M}_{\chi}$ over $A . \Gamma\left(A, \mathcal{M}_{\chi}\right)$ is a free $\mathcal{R}$-module of rank $d$. Choose a basis $\left(e_{1}, \ldots, e_{d}\right)$ in this module. The $(1, x)$-homogeneous component of $\theta$ can be written in this basis as

$$
\xi_{x}=\sum c_{i}(x) e_{i}^{(x)} .
$$

Now define $\psi(x)=\min \operatorname{val} c_{i}(x)$. In the same way as in Section 2.8 , the function $\psi$ defines

1. a pointed cell decomposition $\left(\Delta_{0}, C_{0}\right)$ of $Q$,

2. an $\mathcal{R}$-subalgebra $R$ in $R_{\eta}$.

As before, after the necessary reductions, take $(P, L)=\left(\operatorname{Proj}_{A} R, \mathcal{O}(1)\right)$. The central fiber is a polarized variety $(P, L)\left[\Delta_{0}, \tau_{0}=1, c_{0}, c_{0}^{t}=1, \mathcal{M}_{0}\right]$ and so is a stable semiabelic variety. The restriction of $\theta_{0}$ to each minimal orbit (i.e. corresponding to a vertex of one of the polytopes $\delta_{i}$ ) is not zero, so our condition on orbits is satisfied. Hence, we have obtained the required extension. The uniqueness follows by the next argument. We have a morphism to $A$ defined over $\mathcal{R}$. Choose a section $\operatorname{Spec} \mathcal{R} \rightarrow A$. Then over this section we have a family of stable toric varieties $P^{\prime}$ and the type of the central fiber $P_{0}^{\prime}$ is the same as the type of $P_{0}$. Hence, the uniqueness of the type of the central fiber, the corresponding subalgebra, and of the family follow by the toric case. This special subcase is completed.

To complete the case 1 , after an étale base change we represent $\left(P_{\eta}, L_{\eta}\right), \Theta_{\eta}$ in the form $(P, L, \Theta)\left[c_{\eta}, c_{\eta}^{t}, \mathcal{M}_{\eta}, \tau_{\eta}, \hat{f}_{\eta}\right]$ with $\tau_{\eta}$ being a collection of functions on $C_{1} / B_{1}(\underline{\mathbb{X}})$ with values in appropriate $\mathbb{G}_{m}$-torsors. In other words, $R_{\eta}$ is a union of the algebras as in the special subcase, appropriately glued.

Since $A / S$ is proper, $c_{\eta}^{t}: \pi_{1} \Delta \rightarrow A_{\eta}$ can be extended to $c^{t}: \pi_{1} \Delta \rightarrow A$ over the whole $\mathcal{R}$. After this, the $\mathcal{R}$ algebra $R$ is constructed formally by exactly the same formulas as in Section 2.8.

Case 2. In this case, we have the Mumford-Faltings-Chai uniformization of $G_{\eta}=A_{\eta}$. Again, we use formally the same formulas. However, the complex $\Delta$ now is $X_{\mathbb{R}} / Y$ and the homologies are the group homologies with respect to 
the $Y$-action, as computed in Section 5.6. The integral-valued function $\psi(x)$ becomes a function defined on finitely many points $\left(1, x_{i}\right)$ - representatives of $X / Y$ in $X$. Now, $\psi(x)$ is propagated to an integral-valued function on the whole $X$ by the cocycle $\tau$ according to the rule $\psi\left(x_{i}+y\right)=\psi\left(x_{i}\right)+\operatorname{val} \tau^{[y]\left(1, x_{i}\right)}$. Recall that the function $\operatorname{val} \tau[y]\left(1, x_{i}\right)$ is a nonhomogeneous quadratic function of $y$ whose homogeneous part is positive definite. The convex hull $\mathrm{ConvHull}_{\psi}$ is an "infinite polytope" in the space $X_{\mathbb{R}} \oplus \mathbb{R}$. Because of the positive-definiteness condition, its lower envelope has finite faces. The projection of this lower envelope gives a $Y$-periodic decomposition of $X_{\mathbb{R}}$ into lattice polytopes, i.e., a cell decomposition of $X_{\mathbb{R}} / Y$.

We can now formally define the algebra $\widetilde{R}$ by the same formulas as before where $\widetilde{R}$ is an $\mathcal{R}$-subring of

$$
R_{\eta}=\oplus_{\chi=(d, x), x \in X, d>0} H^{0}\left(A, \mathcal{M}_{\chi}\right) \otimes \mathcal{K} .
$$

Although $R$ is not finitely generated over the $\mathcal{O}_{A}$-algebra itself, $\operatorname{Proj}_{A} R$ is covered by $\operatorname{Spec}_{A}$ 's of finitely generated algebras. Hence, $(\widetilde{P}, \widetilde{L})=\left(\operatorname{Proj}_{A} \widetilde{R}, \mathcal{O}(1)\right)$ is a polarized scheme which is locally of finite type over $A$.

After the reductions, the rest proceeds as in Mumford's construction; see $[\mathrm{FC}],[\mathrm{AN}]$. For the central fiber we have the proper variety $\left(P_{0}, L_{0}\right)=$ $\left(\widetilde{P}_{0}, \widetilde{L}_{0}\right) / Y$ and it is a stable semiabelic variety. It comes with a section $\theta_{0}$ which gives a divisor satisfying our condition of not containing orbits. For any infinitesimal neighborhood $\mathcal{R} / I^{n+1}$ we similarly have the pair $\left(\widetilde{P}_{n}, \widetilde{L}_{n}\right)=$ $(\widetilde{P}, \widetilde{L}) \underset{\mathcal{R}}{\otimes} / I^{n+1}$ and the quotient $\left(P_{n}, L_{n}\right)=\left(\widetilde{P}_{n}, \widetilde{L}_{n}\right) / Y$ is proper over $\operatorname{Spec} \mathcal{R} / I^{n+1}$. The formal scheme defined by the collection $\left\{\left(P_{n}, L_{n}\right)\right\}$ is uniquely algebraizable by a fundamental theorem of Grothendieck to a polarized scheme $(P, L)$ over $\mathcal{R}$. All the infinitesimal thickenings $\left\{\left(P_{n}, L_{n}\right)\right\}$ come with compatible sections $\theta_{n}$ which algebraize to a section $\theta$ of $L$. Finally, we recover $\left(P_{\eta}, L_{\eta}, \theta_{\eta}\right)$ as the generic fiber of $(P, L, \theta)$. This proves the existence of the extension.

By Grothendieck's formal algebraization theorem the family $(P, L, \Theta)$ is equivalent to a system $\left\{\left(P_{n}, L_{n}, \Theta_{n}\right)\right\}$ over $\mathcal{R} / I^{n+1}$. So, to prove the uniqueness, it is sufficient to prove the uniqueness of this system. By Section 4 it defines a system $\left\{\left(\widetilde{P}_{n}, \widetilde{L}_{n}, \widetilde{\Theta}_{n}\right)\right\}$ with a $Y$-action. By the finite case, this system uniquely defines a decomposition $\widetilde{\Delta}$ and the graded $\mathcal{O}_{A}$-algebra $\widetilde{R}=\oplus \tilde{f}_{*} \widetilde{L}^{d}$ together with the $Y$-action. This gives the uniqueness.

Definition 5.7.2. The cell decomposition $\Delta_{0}$ of $X_{\mathbb{R}} / Y$ in the second case was defined, similarly to the finite case, by a lifting property. Accordingly, we will call such decompositions regular.

5.7.3. We note one special case, when $Y=X$. A cell decomposition of $X_{\mathbb{R}} / X$ in this case is the same as an $X$-periodic decomposition of $X_{\mathbb{R}}$ with vertices in $X$. A regular decomposition in this case is the one defined by the 
following lifting property: it is the projection of the lower envelope of the convex hull of the points $\{(x, a(x)) \mid x \in X\}$, where $a(x)$ is a nonhomogeneous quadratic function with a positive-definite homogeneous part. Such decompositions have been known for at least a hundred years. They are usually called the Delaunay decompositions.

Remark 5.7.4. Note that if $\Delta_{1}$ and $\Delta_{2}$ are the complexes for the special, resp. generic, fibers then $\left|\Delta_{1}\right|$ and $\left|\Delta_{2}\right|$ can be identified, providing we are working over $\mathbb{Z}[1 / d]$ and the characteristic of the residue field $\mathcal{R} / m$ does not divide $d$.

5.8. The modified complex $\mathbb{M}_{*}$. Let $(\Delta, C)$ be a pointed complex of lattice polytopes referenced by $\bar{X}_{\mathbb{R}} / Y$. As usual, we represent it as the quotient $\left(\Delta^{\dagger}, C^{\dagger}\right) / Y$ for some $Y \subset X$. We also choose representatives $\left\{\delta_{i}\right\}$ in $\Delta^{\dagger}$ of the maximal cells of $\Delta$; there are finitely many of them. We will only construct the complex $S \mathbb{M}_{*}$ and construct the moduli space in the next section in the case when $\rho:\left|\Delta^{\dagger}\right| \rightarrow X_{\mathbb{R}}$ is injective and the image is convex. An argument similar to 2.10 .1 shows that this is the property which is constant in connected families. In this special case one has $\pi_{1} \Delta=Y$.

Definition 5.8.1. We will define the complex $S C_{*}(\underline{\mathbb{X}})$ in degrees $0,1,2$ in the same way as in the finite case but with the following modifications:

1. The chain group $S C_{1}(\underline{\mathbb{X}})$ will as generators have symbols $\left(\bar{\chi}, \chi_{i_{0}}\right)$, where $\bar{\chi}=\sum y_{s} \cdot \chi_{i_{s}}$ such that $\bar{\chi}$ and $\chi_{i_{0}}$ have the same image in Cone $\Delta^{\dagger} \subset X_{\mathbb{R}}$. We will also add generators $\left(y \cdot \bar{\chi}, y \cdot \chi_{i_{0}}\right)=\left(\bar{\chi}, \chi_{i_{0}}\right)$.

2. The groups $\underline{\text { Fun }}_{\geq 0, i}$ will be replaced as follows. For each $x \in C_{i}$ in a polytope $\delta_{i}$ instead of just having the group $\mathbb{Z}$ we will take $\mathbb{Z} \oplus \mathbb{N}^{d-1}$ ( $d$ will be $h^{0}(\mathcal{M})$ for the ample sheaf on the abelian part). For each $x \in X \cap \delta_{i} \backslash C_{i}$ we will take $\mathbb{N}^{d}$.

We should explain the form of the modified groups $\underline{\text { Fun }}_{\geq 0, i}$. In each space $H^{0}\left(A, \mathcal{M}_{\chi}\right)$ we will choose a basis. With such a basis, an element of this space is the same as a semigroup homomorphism from $\mathbb{N}^{d}$ to the semigroup $k$ for multiplication. For $x \in C_{i}$ the condition is that the section not be 0 . The corresponding parameter space is $\mathbb{A}^{d} \backslash 0$ and is not affine. As usual, we will cover it by $d$ affine sets and these will correspond to the semigroups $\mathbb{Z} \oplus \mathbb{N}^{d-1}$.

LEMMA 5.8.2. With these modifications, the semigroups $S C_{1} / S B_{1}(\underline{\mathbb{X}})$ and $S \mathbb{M}_{1} / B_{1}\left(S \mathbb{M}_{*}\right)$ are finitely generated and finitely presented.

Proof. Let us start with $S C_{1} / S B_{1}(\underline{\mathbb{X}})$. It follows from the relations that the group is generated by the symbols $\left(y_{1} \cdot \chi_{i_{1}}+y_{2} \cdot \chi_{i_{2}}, \chi_{i_{0}}\right)$ with $\rho\left(y_{1} \cdot \chi_{i_{1}}\right)+$ $\rho\left(y_{2} \cdot \chi_{i_{2}}\right)=\rho\left(\chi_{i_{0}}\right)$ and with $\chi_{i_{0}}, \chi_{i_{0}}, \chi_{i_{0}}$ from a fixed finite set of generators for 
the corresponding $\mathbb{X} \cap$ Cone $\delta$. Choose an arbitrary norm on $X_{\mathbb{R}}$. We claim that if some $y$ in such a generator is "large" with respect to this norm then it can be represented as a sum of generators with "shorter" $y$ 's. (This statement is easy to quantify in terms of the sizes of $\delta_{i}$ 's but we will not do it.) For the fixed $i_{0}, i_{1}, i_{2}, y_{1}, y_{2}$ we get semigroups of integral points in finitely generated rational cones, hence finitely generated. Therefore the whole group is finitely generated. As we had mentioned earlier, it is automatic that it is also finitely presented.

To prove our claim, first of all take a very large $\chi_{i_{0}}^{\prime}$ in Cone $\delta_{i_{0}}$. Then

$$
\begin{aligned}
\left(y_{1} \cdot \chi_{i_{1}}+y_{2} \cdot \chi_{i_{2}}, \chi_{i_{0}}\right)= & \left(y_{1} \cdot \chi_{i_{1}}+y_{2} \cdot \chi_{i_{2}}+2 \chi_{i_{0}}^{\prime}, \chi_{i_{0}}+2 \chi_{i_{0}}^{\prime}\right) \\
= & \left(y_{1} \cdot \chi_{i_{1}}+\chi_{i_{0}}^{\prime}, y_{1}^{\prime} \chi_{i_{1}}^{\prime}\right)+\left(y_{2} \cdot \chi_{i_{1}}+\chi_{i_{0}}^{\prime}, y_{2}^{\prime} \chi_{i_{2}}^{\prime}\right) \\
& +\left(y_{1}^{\prime} \cdot \chi_{i_{1}}^{\prime}+y_{2}^{\prime} \cdot \chi_{i_{2}}^{\prime}, y_{3} \chi_{i_{3}}\right) .
\end{aligned}
$$

I now claim that with the restraints on the sizes of $\chi$ 's (they are from a finite set of generators) it is truly obvious that the sizes of $y_{1}, y_{2}$, if one of them is big, are roughly equal, and the sizes of the new $y_{1}^{\prime}, y_{2}^{\prime}, y_{3}$ are roughly half of that (just make a picture). This proves the statement for $S C_{1} / S B_{1}(\underline{\mathbb{X}})$. For $S \mathbb{M}_{1} / B_{1}\left(S \mathbb{M}_{*}\right)$ note that with the relations that we have adding Fun adds several copies of $\mathbb{Z}$ and $\mathbb{N}$, not more than $d$ times the rank of $H_{0}(\underline{\text { Fun }})$, a finite number.

Lemma 5.8.3. The action of the torus $\operatorname{Spec} \mathbb{Z}\left[\mathrm{SM}_{0}\right]=\operatorname{Spec} \mathbb{Z}\left[C_{0}(\underline{\mathbb{X}})\right]$ on the scheme $\mathrm{Spec} \mathbb{Z}\left[\mathrm{SM}_{1} / B_{1}\left(\mathrm{SM}_{*}\right)\right]$ is proper with finite stabilizers.

Proof. This is exactly the same as that of 2.9.7.

Definition 5.8.4. For each $\Delta^{\prime} \geq \Delta$ and $\left(\Delta^{\prime}, C^{\prime}\right) \geq(\Delta, C)$ we have ideals $I\left[\Delta^{\prime}\right]$ and $I\left[\Delta^{\prime}, C^{\prime}\right]$ in $\mathbb{Z}\left[\mathrm{SM}_{1} / B_{1}\left(\mathrm{SM}_{*}\right)\right]$ defined as in the finite case.

5.9. Test families of stable semiabelic pairs. For each decomposition $\Delta^{\prime}$ of $\bar{X}_{\mathbb{R}}^{\prime} / Y^{\prime}$ with $\operatorname{dim} X^{\prime} \leq g$ we are going to construct a complete Artin stack with a universal family of $g$-dimensional stable semiabelic pairs over it. In the end, this stack will be a completion of an open substack in our moduli stack along the stratum corresponding to $\Delta^{\prime}$.

A. The totally degenerate case: $\operatorname{dim} \Delta^{\prime}=g$. Over

$$
U=\operatorname{Spec} \mathbb{Z}\left[S \mathbb{M}_{1} / B_{1}\left(S \mathbb{M}_{*}\right)\right]
$$

there exists a natural family $\left(P^{\dagger}, \Theta^{\dagger}\right)_{U}$ of pairs with the $T$-action. The schemes $P_{U}^{\dagger}$ here are locally of finite type. These are would-be $Y$-covers of the real family $(P, \Theta)_{U}$. The family $\left(P^{\dagger}, \Theta^{\dagger}\right)_{U}$ is constructed in a natural way since the semigroup $S \mathbb{M}_{1} / B_{1}\left(S \mathbb{M}_{*}\right)$ is precisely the semigroup of relations one has to have in such a family. For each cell $\delta$ we consider the semigroup algebras $\mathbb{Z}[$ Cone $\delta]$ and $\mathbb{Z}[$ Cone $y . \delta]$ for all its $Y$-translates. These cover the algebra graded by the 
lattice $\mathbb{X}$ with a representative in each degree $(d, x)$ with $d>0$. A ring homomorphism $\mathbb{Z}\left[S \mathbb{M}_{1}\right] \rightarrow D$ is precise and allows us to glue the rings $D[$ Cone $y . \delta]$ together and to give an element $\theta^{\dagger}$ in degree 1 . The condition for us to actually get a ring is that this homomorphism factors through $\mathbb{Z}\left[S \mathbb{M}_{1} / B_{1}\left(S \mathbb{M}_{*}\right)\right]$.

Hence we have a "universal" $\mathbb{X}$-graded algebra $R_{U}^{\dagger}$ and we define $\left(P^{\dagger}, L^{\dagger}\right)_{U}$ as $\left(\operatorname{Proj}_{U} R^{\dagger}, \mathcal{O}(1)\right)$ which comes with a relative Cartier divisor $\Theta_{U}^{\dagger}$ defined by the section $\theta_{U}^{\dagger}$ of $L_{U}^{\dagger}$. By its very definition, $\left(P^{\dagger}, L^{\dagger}, \theta^{\dagger}\right)_{U}$ also comes with the $Y$-action, and our next aim is to define the quotient. The map $P_{U}^{\dagger} \rightarrow U$ is only locally of finite type and many of the fibers are simply isomorphic to $T$, so that this is just a bit delicate.

An ideal $I[\Delta] \subset \mathbb{Z}\left[S \mathbb{M}_{1} / B_{1}\left(S \mathbb{M}_{*}\right)\right]$ defines $\operatorname{Strat}[\Delta]_{U}$ over which all fibers have type $\Delta$. Over this stratum the $Y$-action is properly discontinuous in Zariski topology, and we can divide the family $\left(P^{\dagger}, \Theta^{\dagger}\right)_{U}$ directly. Now, if we are working over $\mathbb{C}$, we can look at a small neighborhood $V$ open in classical topology over which the $Y$-action on the family is properly discontinuous. Over $V$ we can take the quotient $(P, L, \theta)_{U}$ and it will be a proper family with an ample sheaf and a section.

In general, the method is the same as the essential trick of Mumford's construction [Mum1]. We look at the thickenings

$$
U_{n}=\operatorname{Spec} \mathbb{Z}\left[S \mathbb{M}_{1} / B_{1}\left(S \mathbb{M}_{*}\right)\right] / I[\Delta]^{n+1}
$$

Over each of these thickenings the $Y$-action on the family $\left(P^{\dagger}, L^{\dagger}, \theta^{\dagger}\right)_{U_{n}}$ is properly discontinuous in Zariski topology, and we can take the quotient $(P, L, \theta)_{U_{n}}$. Each sheaf $L_{U_{n}}$ is relatively ample, hence we have a compatible system of projective polarized varieties. By Grothendieck's formal existence theorem [EGA, 5.4.5] this family is equivalent to a projective polarized family $(\widehat{P}, \widehat{L})_{U}$ over the completion $\widehat{U}$. The sections $\theta_{U_{n}}$ glue to a section $\widehat{\theta}$ of $\widehat{L}$. Also, the family $\left(P^{\dagger}, L^{\dagger}, \theta^{\dagger}\right)_{U_{n}}$ comes with a choice of origins for each of the finitely many cells $\delta_{i_{0}}$. Choose one of these sections, say $e$, and look at an open subscheme of $P^{\dagger}$ given by the condition that on every fiber $P_{s}^{\dagger}$ it is the union of components of the smooth locus containing y.e(s). This open subscheme is the $T$-orbit of $\{y . e(s)\}$ and it is a group scheme over $U$. After completion and division by $Y$, it gives a semiabelian group scheme $\widehat{G}$ over $\widehat{U}$ acting on $\widehat{P}_{U}$.

Our last step is to divide $U$ and $\widehat{U}$ by the equivalence relation $R$ given by choosing different origins and by a finite group of symmetries of $\Delta$. On every $U_{n}$ this is given by a torus action of $\operatorname{Spec} \mathbb{Z}\left[S \mathbb{M}_{0}\right] \times U_{n}$. This equivalence relation has finite stabilizer since the equivalence relation $R$ on $U$ has finite stabilizers by Lemma 5.8.3. Finally, we divide the family by the action of a finite group $\operatorname{Sym} \Delta$. The system $\left[U_{n} / R_{n}\right]$ gives a complete Artin stack which we will denote $\mathcal{M}[\Delta]$. It comes with a universal family $(\mathcal{P}, \Theta)$. 
B. The general case. We merely repeat the previous construction but over an appropriate stack classifying the moduli of the abelian part. We start with the stack parametrizing semiabelian varieties with abelian parts of constant rank $r$ and toric parts of constant rank $r$. We take the stack $A_{a, d}$ for the abelian part $(A, \lambda)$. Over it we take $\operatorname{Hom}_{\mathbb{Z}}^{\prime}\left(Y^{\prime} \times X^{\prime}, A \times A^{t}\right)$ which means the closed subscheme of $\operatorname{Hom}_{\mathbb{Z}}\left(Y^{\prime}, A\right) \times \operatorname{Hom}_{\mathbb{Z}}\left(X^{\prime}, A^{t}\right)$ that corresponds to our basic commuting condition $c \circ i=\lambda \circ c$. This is the parameter space for the pairs $\left(c, c^{t}\right)$. Then we add a direct summand $\mathbf{P} \mathbf{i c}^{\lambda}$ parametrizing the rigidified sheaf $\mathcal{M}$. Over this family we consider the functions on $\mathbb{M}_{1} / B_{1}\left(\mathbb{M}_{*}\right)$ with the values in the appropriate torsors. These are exactly what universally describes the graded algebra $R^{\dagger}$ over the abelian part together with a section $\theta^{\dagger}$ of degree 1. At this point, we have a universal family of what would be "universal covers" $\left(P^{\dagger}, \Theta^{\dagger}\right)$ together with the $Y^{\prime}$-action. We finish by applying the same completion / dividing by $Y^{\prime}$ / algebraization construction as before.

Finally, we divide the family by three equivalence relations:

1. by the action of the torus $\operatorname{Spec} \mathbb{Z}\left[\mathbb{M}_{0}\right]$,

2. by the action of $A$ corresponding to changing the origin in the abelian part,

3. finally, by the symmetries of $\bar{X}_{\mathbb{R}}^{\prime} / Y^{\prime}$ and the pairs $(A, \lambda)$.

The first of these equivalence relations is given by a proper action with finite stabilizers. This follows as before by Lemma 5.8.3. The second action is also proper and with finite stabilizers. The third equivalence relation is finite. The outcome is a stack $\mathcal{M}\left[\Delta^{\prime}\right]$ with a "universal" family of stable semiabelic pairs with an arbitrary abelian part.

\subsection{Moduli of stable semiabelic pairs.}

Theorem 5.10.1. 1. The component $\overline{\mathcal{A P}}_{g, d}$ of the moduli stack of stable semiabelic pairs containing $\mathcal{A P}_{g, d}$ and pairs of the same numerical type is a proper Artin stack with finite stabilizers;

2. It has a coarse moduli space $\overline{\mathrm{AP}}_{g, d}$ as a proper algebraic space.

Proof. First, we claim that we can describe all infinitesimal deformations of a pair $\left(G_{0}, P_{0}, \Theta_{0}\right) /$ Spec $k$. Indeed, let $(G, P, \Theta) / S$ be any infinitesimal family which has this pair as the central fiber, with local artinian $S$. Then the ranks of abelian and toric parts are constant, and $G / S$ is a global extension of $A / S$ by $T / S$. By taking an étale cover we can make the toric part to be split. By the results of Section 4 we have, in a canonical way, a family $(G, \widetilde{P}, \widetilde{\Theta})$ with the sheaf $\widetilde{L}=\mathcal{O}(\widetilde{\Theta}) T$-linearized. Let $\left(G, P^{\dagger}, \Theta^{\dagger}\right)$ be a connected component. 
By choosing a section we have a projection $p$ to the abelian part. Any finite union of irreducible components $(G, P, \Theta)_{f}$ of $\left(G, P^{\dagger}, \Theta^{\dagger}\right)$ is a stable semiabelic pair. By 2.5.1 the higher direct images $R^{i} p_{f *} L_{f}$ vanish. We conclude that describing any finite part of $\left(G, P^{\dagger}, \Theta^{\dagger}\right)$ is equivalent to describing the graded $\mathcal{O}_{A^{-}}$-algebra $R_{f}=\oplus_{d \geq 0} L_{f}^{d}$ with an element $\theta_{f}$ of degree 1 . Hence, describing the family $\left(G, P^{\dagger}, \Theta^{\dagger}\right)$ itself is equivalent to describing a graded $\mathcal{O}_{A}$-algebra $R^{\dagger}$ with an element $\theta^{\dagger}$ of degree 1 . Because of the universal character of our relations in $S \mathbb{M}_{1} / B_{1}\left(S \mathbb{M}_{*}\right)$ we see that the family $(P, \Theta) / S$ is the pullback of the universal family under a unique morphism from $S$ to the stack $\mathcal{M}[\Delta]$.

At this point, we are ready to apply Theorem 5.3 of [Art1]; cf. the case of surfaces of general type [Art2, 5.5] there. Our groupoid of families of stable semiabelic pairs has a deformation theory and obstruction theory (even better than that) and for any point we have a pro-representable family of projective objects. Each pair $(P, \Theta)$ has a finite automorphism group. The openness of versality follows from the fact that for $\Delta^{\prime} \leq \Delta$ the semigroup model $\left[\operatorname{Spec} \mathbb{Z}\left[S \mathbb{M}_{1}\right] / B_{1}\left(S \mathbb{M}_{*}\right) / \operatorname{Spec} \mathbb{Z}\left[\mathbb{M}_{0}\right]\right]$ for $\Delta^{\prime}$ is an open substack of the semigroup model for $\Delta$. Hence, all conditions of Artin's method are satisfied and we have an algebraic stack in Artin's sense. Our stack is complete by Section 5.7. Also, the formal models we have constructed are separated. Since every 1-parameter family can be completed and the type of the central fiber in a 1-parameter family is uniquely defined, our stack is also proper. It has a coarse moduli space as a proper algebraic space by $[\mathrm{KM}]$.

5.11. Approximation of the moduli space. Similarly to Section 2.11, we are now going to describe our moduli space in more detail in the case of the complex $\bar{X}_{\mathbb{R}} / X$, i.e. the component containing principally polarized abelian torsors. We shall construct a simplification of our moduli space and identify the main irreducible component with a particular toroidal compactification of $A_{g}$. This we will do in the totally degenerate case, over the 0-cusp only. The general case at this point would not add anything but bookkeeping complications.

In the lattice $C_{0}\left(\bar{X}_{\mathbb{R}} / X, \underline{\text { Fun }}\right) / B_{0}\left(\bar{X}_{\mathbb{R}} / X, \underline{\mathbb{L}}\right)$, which by Lemma 5.1 .5 is a lattice of dimension $g(g+1) / 2+(g+1)$, consider an infinite "polytope" $\sigma$ with vertices $1_{x}, x \in X$. This is an analog of a simplex from the finite case in Section 2.11. There is a natural projection $\phi: \sigma \rightarrow(1, X)$ and the groups we are about to define will be constructed from the fibers of this "polytopal" map.

5.11.1. For every $q \in X_{\mathbb{R}}$ we define the convex figure $\sigma_{q}$ as the fiber of $\sigma \rightarrow X$ over $(1, q)$. This is an infinite "polytope" of dimension $g(g+1) / 2$ lying in the space parallel to $H^{0}\left(\bar{X}_{\mathbb{R}} / X, \underline{\mathbb{L}}\right)$ and, similar to the finite case, it has an explicit description as follows. Let $\delta$ be a $k$-dimensional $(0 \leq k \leq g)$ simplex fitting in $X_{\mathbb{R}} / X$, i.e. whose $X$-translates intersect $\delta$ only at faces which contains 
$q$ in its interior. Say that $\delta$ has vertices $x_{0}, \ldots x_{k}$ and $(1, q)=\sum c_{i}\left(1, x_{i}\right)$. Then there is a vertex of $\sigma_{q}$ corresponding to $\delta$ which is an element of $C_{0}(\underline{\text { Fun }}) / B_{0}(\underline{\mathbb{L}})$ of the form

$$
\frac{1}{k+1} \sum c_{i} 1_{x_{i}}
$$

Vertices of $\sigma_{q}$ correspond in a 1-to-1 way to simplices in $\bar{X}_{\mathbb{R}} / X$ whose translation class contains $q$ in the interior, and higher-dimensional faces correspond to nonsimplicial cells with this property. The dual fan $\operatorname{Fan}\left(\sigma_{q}\right)$ divides $\operatorname{Sym}^{2} X_{\mathbb{R}}^{*}$, i.e. the space of quadratic functions on $X_{\mathbb{R}}$, into cones according to whether their Delaunay decompositions contain the same cell with $q$ in the interior.

Definition 5.11.2. For a quadratic function $\psi$ on $X$ consider the lower envelope of the points $(x, \psi(x)) \in X_{\mathbb{R}} \times \mathbb{R}$ (this only makes sense if $\psi$ is semipositive definite). The projection of this lower envelope is called the Delaunay decomposition of $\psi$.

5.11.3. Now consider the Minkowski sum $\Sigma\left(\bar{X}_{\mathbb{R}} / X\right)$ of several $\sigma_{\alpha}$. The only condition is that we should have enough points $\alpha$ so that they can distinguish any two Delaunay triangulations according to which cells contain them (it is enough to consider the points appearing as centers of simplices, and these are among the finitely many, modulo $X$, rational points with denominator $(r+1)$ !). Going through the same arguments with Minkowski averages as in Section 2.12, we see that the faces of $\Sigma\left(\bar{X}_{\mathbb{R}} / X\right)$ are in 1-to-1 correspondence with Delaunay decompositions, and that the dual fan $\operatorname{Fan}\left(\Sigma\left(\bar{X}_{\mathbb{R}} / X\right)\right)$ divides the cone of semipositive definite symmetric quadratic forms into cones according to whether they define the same Delaunay decomposition. This is precisely the classical definition of the second Voronoi decomposition.

Definition 5.11.4. Now, let $\Delta$ be a triangulation of $\bar{X}_{\mathbb{R}} / X$ into lattice polytopes. For every $q$ we can define a saturated semigroup $K_{\delta, q}$ in $H^{0}(\underline{\mathbb{L}})$ defined by differences $\chi_{1}-\chi_{2}$ with $\chi_{1} \in$ Cone $\sigma, \chi_{2} \in$ Cone $\delta, \phi\left(\chi_{1}\right)=\phi\left(\chi_{2}\right)=$ $N(1, q)$ for some cell $\delta$ of $\Delta$ with $q \in \delta^{0}$. This gives an affine $\operatorname{Spec} K_{\delta, q}$.

The group $K_{\delta, q}$ is one of the groups associated with the infinite "polytope" $\sigma_{q}$. The advantage is that the former is a semigroup of integral vectors in a finite rational polyhedral cone. Note that for $x \in X$ the groups $K_{q, \delta}$ and $K_{q+x, \delta+x}$ are naturally identified because

$$
\left(y \cdot \chi_{1}-y \cdot \chi_{2}\right)-\left(\chi_{1}-\chi_{2}\right) \in B_{0}(\underline{\mathbb{L}}) .
$$

As in the finite case, once we have chosen a decomposition $\Delta$, there is a finite decomposition of $\bar{X}_{\mathbb{R}} / X$ into polytopal regions according to the type of a fiber $K_{\delta, q}$. Hence, we can define

$$
U[\Delta]=\lim _{\longleftrightarrow} \operatorname{Spec} \mathbb{Z}\left[K_{\delta, \alpha}\right]=\operatorname{Spec} \mathbb{Z}\left[\lim K_{\delta, \alpha}\right] .
$$


LEMMA 5.11.5. There is a finite morphism from the coarse moduli space $M[\Delta]$ of the stack $\mathcal{M}[\Delta]$ to the completion of $U[\Delta]$ along $\operatorname{Strat}[\Delta]$.

Proof. It is sufficient to check this on the semigroup models before the completion, for the morphism

$$
\operatorname{Spec} \mathbb{Z}\left[\operatorname{ker}\left(S \mathbb{M}_{1} / B_{1}\left(S \mathbb{M}_{*}\right) \rightarrow \mathbb{M}_{0}\right] \rightarrow U[\Delta]\right.
$$

Here is how this map is constructed. Over $\operatorname{Spec} \mathbb{Z}\left[S \mathbb{M}_{1} / B_{1}\left(S \mathbb{M}_{*}\right)\right]$ we have a family $\left(P^{\dagger}, L^{\dagger}, \theta^{\dagger}\right)$. Write $\theta^{\dagger}=\sum_{X} \xi_{x}$ as the infinite sum of its homogeneous components. For every finite collection $\left(n_{x}\right) \in \operatorname{Fun}\left(X, \mathbb{Z}_{\geq 0}\right)$ we have an element $\prod \xi_{x}^{n_{x}}$, and for two such products in the same homogeneous space, we can compare them. The products with $\left(n_{x}\right) \in \operatorname{Cone} \delta$, for $\delta \in \Delta$, generate the corresponding 1-dimensional eigenspace $R_{\chi}$. Hence, we can write

$$
\prod \xi_{x}^{m_{x}}=c\left(\left(m_{x}\right),\left(n_{x}\right)\right) \prod \xi_{x}^{m_{x}}
$$

Because $L^{\dagger}$ and $\theta^{\dagger}$ are invariant under the $Y=X$-action, one has

$$
c\left(y \cdot\left(m_{x}\right), y \cdot\left(n_{x}\right)\right)=c\left(\left(m_{x}\right),\left(n_{x}\right)\right) .
$$

Hence, we have a coefficient $c$ defined for every pair of integral vectors in the same fiber of Cone $(\sigma) \rightarrow \mathbb{X}$. As in the finite case, these coefficients $c$ define a homomorphism $K_{\delta, q} \rightarrow \mathbb{Z}\left[S \mathbb{M}_{1} / B_{1}\left(S \mathbb{M}_{*}\right)\right]$ and by taking the limit we have the required homomorphism $\operatorname{Spec} \mathbb{Z}\left[S \mathbb{M}_{1} / B_{1}\left(S \mathbb{M}_{*}\right)\right] \rightarrow U[\Delta]$. Finally, note that this construction does not depend on the choice of origins, and so we obtain the required homomorphism.

As in the finite case, both spaces have natural stratification by decompositions $\Delta^{\prime} \geq \Delta$ and the strata, which are respectively Spec $\mathbb{Z}\left[H^{1}\left(\Delta^{\prime}, \mathbb{M}_{*}\right)\right]$ and $\operatorname{Spec} \mathbb{Z}\left[H^{0}\left(\Delta^{\prime}, \underline{\mathbb{L}}\right)\right]$, are isogenic.

As $\Delta$ goes over all triangulations of $\bar{X}_{\mathbb{R}} / X$, the completions of $U[\Delta]$ cover the simplification $M_{\text {simp }}$ of our moduli $\overline{\mathrm{AP}_{g}}$, and we obtain a finite morphism $\overline{\mathrm{AP}_{g}} \rightarrow M_{\text {simp }}$

THEOREM 5.11.6. The toroidal compactification of $A_{g}$ for the second Voronoi decomposition is isomorphic to the main irreducible component of $\overline{\mathrm{AP}}_{g}$, the one containing $\mathrm{A}_{g}=\mathrm{AP}_{g}$.

Proof. Again, we check this on the level of semigroup models, before the completion. The main component of $\overline{\mathrm{AP}}_{g}$ maps to $M_{\text {simp }}$. On the other hand, for every Delaunay triangulation $\Delta$ there is a cone Cone $[\Delta]$ in $\operatorname{Fan}\left(\Sigma\left(\bar{X}_{\mathbb{R}} / X\right)\right)$, i.e. the second Voronoi decomposition with a natural surjective map $\underset{\longrightarrow}{\longrightarrow} K_{\delta, q} \rightarrow$ Cone $[\Delta]$. 
This means that the main component of $M_{\text {simp }}$ is already normal and is isomorphic to the toroidal compactification of $A_{g}$ for the second Voronoi compactification. We have a finite map $\overline{\mathrm{AP}}_{g} \rightarrow M_{\text {simp }}$ which is an isomorphism on the main stratum, the one corresponding to $\bar{X}_{\mathbb{R}} / X$. Hence the main irreducible component of the space $\overline{\mathrm{AP}}_{g}$ is the same.

5.12. Generalized secondary polytopes for periodic decompositions. For a polytopal decomposition of $\bar{X}_{\mathbb{R}} / Y$ (where $Y \subset X$ has finite index) the definition of the secondary polytope $\Sigma(\Delta)$ is a straightforward generalization of that for the finite case which we considered in Section 2.12:

Definition 5.12.1. $\Sigma(\Delta)$ is defined as the image of $\prod \Sigma\left(\delta_{i}\right) \subset C_{0}\left(\Delta, \underline{\operatorname{Fun}}_{\mathbb{R}}\right)$ in $C_{0}\left(\Delta, \underline{\operatorname{Fun}}_{\mathbb{R}}\right) / B_{0}\left(\Delta, \underline{\mathbb{L}}_{\mathbb{R}}\right)$, where $\delta_{i}$ go over maximal-dimensional (i.e. minimal by the reverse of inclusion order) cells.

Example 5.12.2. Let $E_{n}$ be the decomposition of $\mathbb{R}^{n}$ into standard Euclidean cubes. There is only one maximal-dimensional cell modulo translation. Then $\Sigma\left(E_{n}\right)$ is simply the image of the secondary polytope of the cube in a lower-dimensional space, of dimension $n(n-1) / 2$ instead of $2^{n}-n-1$.

Similar to Section 2.12, faces of this secondary polytope are in 1-to-1 correspondence with regular subdivisions of $\Delta$.

For an arbitrary decomposition of $\bar{X}_{\mathbb{R}} / Y$ we can define $\Sigma(\Delta)$ as an appropriate Minkowski sum of "polytopes" some of which are infinite, just as we have done in the previous section for $\bar{X}_{\mathbb{R}} / X$. This gives an "unbalanced" version of $\Sigma(\Delta)$. Below we construct a balanced, $\mathrm{GL}(X)$-invariant version combinatorially equivalent to the above, and giving the same dual fan.

5.12.3. For every $q \in X_{\mathbb{R}}$ we define the balanced convex figure $\delta_{q}^{\text {bal }}$ as follows. Let $\delta$ be a $k$-dimensional $(0 \leq k \leq r)$ simplex fitting in $X_{\mathbb{R}} / X$, i.e. whose $X$-translates intersect $\delta$ only at faces which contain $q$ in their interiors. Consider all the $k+1$ translates $\delta^{0}, \ldots, \delta^{k}$ of it that contain the origin 0 , and let $q_{0}, \ldots, q_{k}$ be the corresponding translates of $q$ in their interiors. Say, $\delta^{j}$ has vertices $x_{0}^{j}, \ldots, x_{k}^{j}$ and $\left(1, q_{i}\right)=\sum c_{i}\left(1, x_{i}^{j}\right)$. Then define the vertex of $\sigma_{q}$ corresponding to $\delta$ as the image in $C_{0}(\underline{\text { Fun }}) / B_{0}(\underline{\mathbb{L}})$ of

$$
\frac{1}{k+1} \sum c_{i} 1_{x_{i}^{j}}
$$

Taking the Minkowski sum of these balanced "polytopes" gives the explicit "balanced" coordinate definition for the secondary polytope obtained by averaging the coordinates of the vertices above, similar to the equation (2) in Definition 2.12.4. 
Definition 5.12.4. For each triangulation $\operatorname{Tr}$ of $\bar{X}_{\mathbb{R}} / X$ let $\phi_{\operatorname{Tr}}$ be an element of $C_{0}(\underline{\text { Fun }}) / B_{0}(\underline{\mathbb{L}})$ defined by the formula

$$
\phi_{\operatorname{Tr}}=\sum_{\delta_{i}: x \in \delta_{i}, 0 \in \delta_{i}} \operatorname{Vol} \delta_{i} \cdot\left(1_{x}-1_{0}\right) .
$$

Then, for any $x \neq 0$,

$$
\phi_{\operatorname{Tr}}(x)=\sum_{\delta_{i}: x \in \delta_{i}, 0 \in \delta_{i}}, \operatorname{Vol} \delta_{i}
$$

and for the origin we have $\phi_{\operatorname{Tr}}(0)=-r(r+1)$ !, which comes from $r=$ number of vertices in a simplex minus one, and $(r+1) !=$ volume of $\operatorname{Star}(0)$ in the lattice measure of volume.

Define $\Sigma\left(\bar{X}_{\mathbb{R}} / X\right)$ as the convex hull of the points $\phi_{\operatorname{Tr}}$ as $\operatorname{Tr}$ goes over all the triangulations of $\bar{X}_{\mathbb{R}} / X$. This is a "polytope" which may (and indeed does) have infinitely many vertices.

LEMMA 5.12.5. $\quad \Sigma\left(\bar{X}_{\mathbb{R}} / X\right)$ is invariant with respect to the action of $\mathrm{GL}(X)$.

Proof. Indeed, for any $g \in \mathrm{GL}(X)$ and any triangulation $\operatorname{Tr}$ one has $g \cdot \phi_{\operatorname{Tr}}=\phi_{g} \cdot \operatorname{Tr}$.

Lemma 5.12.6. $\Sigma\left(\bar{X}_{\mathbb{R}} / X\right)$ lies in the subspace $H_{0}(\underline{\mathbb{L}})=C_{0}(\underline{\mathbb{L}}) / B_{0}(\underline{\mathbb{L}})$.

Proof. We need to check that the vertices $\phi_{\operatorname{Tr}}$ in $C_{0}(\underline{\text { Fun }})$ have the same image when projected to $C_{0}(\underline{\mathbb{X}})=\mathbb{X}$. Consider one such vertex corresponding to a triangulation $\operatorname{Tr}$. Let $\sigma_{1}$ be one of the maximal-dimensional simplices in this triangulation. In the translation equivalence class of $\sigma$ there are precisely $r+1$ simplices that contain 0 : they are obtained by shifting one of the $r+1$ vertices to the origin. For every edge $\{0, x\}$ in one of these $r+1$ simplices there is precisely one edge $\{0,-x\}$ in its copy shifted by $(-x)$. Therefore, all terms in the definition of $\phi_{\operatorname{Tr}}$ split into groups of the form $\left(1_{x}+1_{-x}-2 \cdot 1_{0}\right)$, and each of these groups has image 0 in $\mathbb{X}$.

Corollary 5.12 .7 (of the proof). When considered as an element of $\operatorname{Sym}^{2} X$,

$$
\phi_{\operatorname{Tr}}=\frac{1}{2} \sum_{\delta_{i}: x \in \delta_{i}, 0 \in \delta_{i}} \operatorname{Vol} \delta_{i} \cdot x \otimes x .
$$

Proof. Indeed, under the isomorphism $H_{0}(\underline{\mathbb{L}}) \stackrel{\sim}{\rightarrow} \operatorname{Sym}^{2} X$, the element $\left(1_{x}+\right.$ $\left.1_{-x}-2 \cdot 1_{0}\right)$ goes to $x \otimes x$.

COROLlary 5.12.8. The toroidal compactification over $\mathbb{Z}$ of $A_{g}$ corresponding to the second Voronoi decomposition is projective. 
Proof. This follows by Tai's criterion in [AMRT] which requires a $\mathrm{GL}(X)$ invariant polarization function on the fan. In our case it is provided by $\Sigma\left(X_{\mathbb{R}} / X\right)$. Even though [AMRT] works over $\mathbb{C}$, the result applies in every case when the Satake-Baily-Borel compactification is constructed as a projective scheme, which is done over $\mathbb{Z}$ in $[\mathrm{FC}]$. See also [Cha, IV] where Tai's criterion is explained over $\mathbb{Z}[1 / 2]$.

5.13. Periodic non-Delaunay decompositions. It is very easy to construct nonregular periodic decompositions when the degree of polarization is big: simply take any finite nonregular decomposition and repeat it periodically filling the holes in an arbitrary periodic pattern. The lifting property will not be satisfied even locally. The principally polarized case is significantly harder. It can be checked by hand that in dimensions $g \leq 3$ all periodic decompositions are regular, i.e. Delaunay. There is an example in dimension 4 , however.

Example 5.13.1. Erdahl and Ryshkov in [ER, 3.4] describe a "red triangular" lattice of dimension 4 which has the following Delaunay decomposition $\Delta$ : two cyclic polytopes $C(4,6)$ (of dimension 4 , with 6 vertices) and 18 simplices $\alpha_{4}$. The two $C(4,6)$ 's are mirror-symmetric with respect to a vertex.

We shall construct a subdecomposition of $\Delta$ which is not Delaunay itself. If each cell is simplicial, so that the proper faces cannot be subdivided any further, the secondary polytope $\Sigma \Delta$ is $\prod \Sigma\left(\delta_{i}\right)$, the direct product of the secondary polytopes of the cells.

That is the case here, because a cyclic polytope is simplicial. Now, $\Sigma\left(\alpha_{4}\right)$ is a point and $\Sigma(C(4,6))$ has dimension $6-4-1=1$ and is a closed interval. The end points correspond to two possible splittings of $C(4,6)$ into three simplices. Therefore, $\Sigma(\Delta)$ is a square and the corresponding toric scheme is $P_{\Sigma(\Delta)}=$ $\mathbb{P}^{1} \times \mathbb{P}^{1}$

There are exactly six nonregular subdecompositions of $\Delta$. They correspond to splittings of the cyclic polytopes which are not mirror symmetric. They are not Delaunay because every Delaunay decomposition has the symmetry $x \mapsto-x$.

The moduli interpretation of this example is that the connected component of $\mathrm{AP}_{4}$ containing $A_{4}$ (the moduli of PPAVs of dimension 4) also contains another irreducible component. It is 2-dimensional.

Example 5.13.2. There is a very elegant description of Delaunay decompositions of root lattices $A_{n}, D_{n}, E_{6,7,8}$ and their duals (weight lattices) in terms of their Coxeter-Dynkin diagrams: it was given in [CS], [MP]; see also [Erd]. In particular, the Delaunay decomposition of the $E_{8}$ lattice consists of 135 crosspolytopes $\beta_{8}$, each generating an index-2 sublattice, and 1920 triplevolume simplices $\alpha_{8}$. 
Since crosspolytopes (see [Cox, 7.2]) are simplicial, the regular subdecompositions of $E_{8}$ are described by the faces of a polytope $\Sigma\left(E_{8}\right)$ which is again the direct product of the secondary polytopes of the cells.

It can be easily checked that the secondary polytope of an $n$-dimensional crosspolytope is an $(n-1)$-dimensional simplex: $\Sigma\left(\beta_{n}\right)=\alpha_{n-1}$, and that the corresponding secondary variety is the projective space $\mathbb{P}^{n-1}$. Therefore, $\Sigma\left(E_{8}\right)$ is the direct product of 135 copies of $\alpha_{7}$, and $P_{\Sigma\left(E_{8}\right)}$ is the direct product of 135 copies of $\mathbb{P}^{7}$.

The moduli interpretation of this example is that the connected component of $\mathrm{AP}_{8}$ containing $A_{8}$ (the moduli of PPAVs of dimension 8) also contains another irreducible component of dimension 945. Recall that $A_{8}$ itself (moduli space $A_{8}$, not the lattice $A_{8}$ ) has dimension 36 .

\section{Further questions}

We list just some of the many questions that arise in connection to our moduli spaces:

1. How should one define level structures for semiabelic pairs that behave well at infinity?

2. In the case of the nonprincipal polarization dimension of the main component of $\mathrm{AP}_{g, d}$, is $(d-1)$ larger than $\operatorname{dim} A_{g, d}$ ? Is there a simple way to cut this dimension back to $\operatorname{dim} \mathrm{A}_{g, d}$ by considering not all pairs but perhaps only some symmetric ones, possibly on a finite cover corresponding to a level structure that behaves well at infinity? The canonical level structure seems to be a natural choice here. What is the right definition of the canonical level structure for the pairs?

3. Is there a way to isolate $\bar{A}_{g}^{\text {Vor }}$ in $\overline{\mathrm{AP}_{g}}$ using a log structure? Is there a good definition for the moduli of pairs with a log structure?

4. According to Mumford and Namikawa [Nam1] the Torelli map $M_{g} \rightarrow A_{g}$ extends to a morphism from the Mumford-Deligne compactification $\bar{M}_{g}$ to $\bar{A}_{g}^{V o r} \subset \overline{\mathrm{AP}_{g}}$. One would expect that with our moduli compactification there must be a functorial map $\bar{M}_{g} \rightarrow \overline{\mathrm{AP}}_{g}$. We show that this is indeed the case in [Ale1].

5. Are the moduli spaces we have constructed always reduced? I suppose that the answer is no.

6. Are they connected, once we fix the numerical type? Again, I think the answer in general may be no. (A recent example of F. Santos [San] shows that the answer is in general no for $M_{Q}$, where $Q$ is a lattice polytope.) 
7. The last question is directly related to the generalized Baues problem in geometric combinatorics. We hope that the geometric constructions of the present paper, in particular, the configuration of the generalized secondary polytopes that we have constructed, may provide new clues for the resolution of this problem.

8. Explicit description of various secondary polytopes describing parts of our moduli spaces is an interesting combinatorial problem in itself.

9. We only considered the semiabelian group action in this paper. What about other algebraic groups?

University of Georgia, Athens, GA

E-mail address: valery@math.uga.edu

REFERENCES

[Ale1] V. Alexeev, Compactified jacobians, preprint, 1996, http://front.math.ucdavis.edu/ alg-geom9608012.

[Ale2] Moduli spaces $M_{g, n}$ for surfaces, 1-22, Proc. Internat. Conf. (Trento, Italy, June, 1994), Walter de Gruyter, Berlin, 1996.

[AMRT] A. Ash, D. Mumford, M. Rapoport, and Y. Tai, Smooth compactification of locally symmetric varieties, in Lie Groups: History, Frontiers and Applications IV, Math. Sci. Press, Brookline, Mass., 1975.

[AN] V. Alexeev and I. NAkamura, On Mumford's construction of degenerating abelian varieties, Tohoku Math. J 51 (1999), 399-420.

[Art1] M. Artin, Algebraization of formal moduli I, in Global Analysis, 21-71 (Papers in honor of K. Kodaira), Princeton Univ. Press, Princeton, NJ, 1969.

[Art2] Versal deformations and algebraic stacks, Invent. Math. 27 (1974), 165189.

[Bac] K. Baclawski, Cohen-Macaulay ordered sets, J. Algebra 63 (1980), 226-258.

[BH] W. Bruns and J. Herzog, Cohen-Macaulay Rings, Cambridge Stud. Adv. Math. 39, Cambridge Univ. Press, Cambridge, 1993.

[BLR] S. Bosch, W. Lütkebohmert, and M. Raynaud, Néron Models, Ergebnisse der Mathematik und ihrer Grenzgebiete 21, Springer-Verlag, New York, 1990.

[Bre] G. E. Bredon, Sheaf Theory, second ed., Grad. Texts in Math. 170, SpringerVerlag, New York, 1997.

[BS] L. J. Billera and B. Sturmfels, Fiber polytopes, Ann. of Math. 135 (1992), 527549.

[Cha] C.-L. Chai, Compactification of Siegel Moduli Schemes, London Math. Soc. Lecture Notes Series 107, Cambridge Univ. Press, Cambridge, 1985.

[Cox] H. S. M. Coxeter, Regular Polytopes, third ed., Dover Publ. Inc., New York, 1973.

[CS] J. H. Conway and N. J. A. Slonne, The cell structures of certain lattices, in Miscellania Mathematica, 71-107, Springer-Verlag, New York, 1991.

[DE] J. Giraud, A. Grothendieck, S. L. Kleiman, M. Raynaud, and J. Tate, Dix Exposés sur la Cohomologie des Schémas, Adv. Studies in Pure Math. 3, Masson \& Cie, Paris; North-Holland, Amsterdam, 1968.

[DW] B. H. Dayton and C. A. Weibel, A spectral sequence for the $K$-theory of affine glued schemes, in Algebraic K-theory (Evanston, 1980) Lecture Notes in Math. 854 (1981), 24-92, Springer-Verlag, New York. 
[EGA] A. Grothendieck and J. A. Dieudonné, EGA III. Étude Cohomologique des Faisceaux Cohérents, Inst. Hautes Études Sci. Publ. Math. 11, 17 (1961), (1963).

[ER] R. M. ERdahl and S. S. Ryshrov, The empty sphere, II, Canad. J. Math. 40 (1988), 1058-1073.

[Erd] R. M. ERdahl, A cone of inhomogeneous second-order polynomials, Discrete Comput. Geom. 8 (1992), 387-416.

[FC] G. Faltings and C.-L. Chai, Degenerations of Abelian Varieties 22, Ergebnisse der Mathematik und ihrer Grenzgebiete, Springer-Verlag, New York, 1990.

[FGA] A. Grothendieck, Technique de descente et théorèmes d'existence en géométrie algébrique. V: Les schémas de Picard: théorèms d'existence. VI: Les schémas de Picard: propriétés générales, Sem. Bourbaki 232, 236 (1962), 1-19, 1-23, Secrétariat Math., Paris.

[Ful] W. Fulton, Introduction to Toric Varieties, Ann. of Math. Studies 131, Princeton Univ. Press, Princeton, NJ, 1993.

[GKZ] I. M. Gelfand, M. M. Kapranov, and A. V. Zelevinsky, Discriminants, Resultants, and Multidimensional Determinants, Birkhäuser, Boston, 1994.

[God] R. Godement, Topologie Algébrique et Théorie des Faisceaux, Hermann, Paris, 1958.

[GT] S. Greco and C. Traverso, On seminormal schemes, Compositio Math. 40 (1980), 325-365.

[KSZ] M. M. Kapranov, B. Sturmfels and A. V. Zelevinsky, Quotients of toric varieties, Math. Ann. 290 (1991), 643-655.

[KM] S. Keel and S. Mori, Quotients by groupoids, Ann. of Math. 145 (1997), 193-213.

[Kol] J. Kollár, Rational Curves on Algebraic Varieties, Ergebnisse der Mathematik und ihrer Grenzgebiete 32, Springer-Verlag, New York, 1996.

[Lan] S. Lang, Abelian Varieties, Interscience Publ. Inc., New York, 1959.

[Mil] J. S. Milne, Étale Cohomology, Princeton Math. Series 33, Princeton Univ. Press, Princeton, NJ, 1980.

[Mor] S. Mori, On a generalization of complete intersections, J. Math. Kyoto Univ. 15 (1975), 619-646.

[MP] R. V. Moody and J. Patera, Voronoi and Delaunay cells of root lattices: classification of their faces and facets by Coxeter-Dynkin diagrams, J. Phys. A. 25 (1992), 5089-5134.

[Mum1] D. Mumford, An analytic construction of degenerating abelian varieties over complete rings, Compositio Math. 24 (1972), 239-272.

[Mum2] _ Geometric Invariant Theory, third ed., Ergebnisse der Mathematik und ihrer Grenzgebiete 34, Springer-Verlag, New York, 1994.

[Nam1] Y. Namikawa, A new compactification of the Siegel space and degeneration of Abelian varieties, I, II, Math. Ann. 221 (1976), 97-141, 201-241.

[Nam2] Toroidal degeneration of Abelian varieties, in Complex Analysis and Algebraic Geometry, 227-237, Cambrdige Univ. Press, Cambridge, 1977.

[Oda] T. OdA, Convex Bodies and Algebraic Geometry, Springer-Verlag, New York, 1988.

[Ray1] M. Raynaud, Faisceaux Amples sur les Schémas en Groupes et les Espaces Homogènes, Lecture Notes in Math. 119 (1970), Springer-Verlag, New York.

[Ray2] Spécialisation du foncteur de Picard, Inst. Hautes Études Sci. Publ. Math. 38 (1971), 27-76.

[San] F. Santos, A point set whose set of triangulations is disconnected, J. A. M. S. 13 (2000), 611-637.

[SGA1] A. Grothendieck, SGA1. Revêtements Étales et Groupe Fondamental, Lecture Notes in Math. 224, Springer-Verlag, New York, 1971.

[SGA3] M. Demazure, A. Grothendeck, et AL., SGA3. Schémas en Groupes, Lecture Notes in Math. 151, 152, 153, Springer-Verlag, New York, 1970. 
[SGA71] A. Grothendieck, M. Raynaud, and D. S. Rim, SGA7 I, Groupes de Monodromie en Géometrie Algébrique, Lecture Notes in Math. 288, Springer-Verlag, New York, 1972.

[Spa] E. H. Spanier, Algebraic Topology, McGraw-Hill Book Co., New York, 1966.

[Sta1] R. Stanley, Combinatorics and Commutative Algebra 41, Birkhauser, Basel, 1985.

[Sta2] Generalized $H$-vectors, intersection cohomology of toric varieties, and related results, in Commutative Algebra and Combinatorics (Kyoto, 1985), Adv. Stud. Pure Math 11 (1987), 187-213.

[Tra] C. Traverso, Seminormality and Picard group, Ann. Scuola Norm. Sup. Pisa 24 (1970), 585-595.

[Yuz] S. Yuzvinsky, Cohen-Macaulay rings of sections, Adv. in Math. 63 (1987), 172-195.

(Received May 21, 1999) 